\title{
Poster Presentation Abstracts, 25th Annual Meeting of the International Association of Medical Science Educators, June 12-17, 2021
}

Published online: 29 November 2021

(c) The Author(s) under exclusive licence to International Association of Medical Science Educators 2021

\section{Assessment}

\section{ANALYSIS OF THE RELATIONSHIP OF MEDICAL STUDENT PERSONAL DISTRESS AND EMOTION REGULATION USING MACHINE LEARNING}

Robert Treat, PhD IJeffery D. Fritz, PhD IAmy Prunuske, $\mathrm{PhD}$ IWilliam J. Hueston, M. D. IKristina Kaljo, PhD ICraig Hanke, PhD IMolly Falk-Steinmetz, MS

Medical College of Wisconsin

PURPOSE: Medical Student Personal Distress (PD) Is a Vicarious, Emotionally Unpleasant Reaction To Another Person's Suffering and Is Derived From the Affective Internal Structure of Empathy. PD Is a Distinctive Factor From Other Emotionally Detached Elements of Empathy Such As Perspective Taking, Which Measures the Cognitive Aspect of Empathy. Recent Reports Indicate a Relationship of PD To Emotion Regulation, ${ }^{1}$ but evidence is conflicting regarding relationships to situational ${ }^{2}$ and dispositional factors. ${ }^{3}$ Machine learning will help resolve analytical conflicts and provide cluster profiles of students with varying levels of empathy. The purpose of this study is to analyze the combined effects of personal distress, Emotion Regulation, and Dispositional Stability Using Machine Learning.

METHODS: In 2017/18, 205/500 M-1/M-2 Medical Students Voluntarily Completed the Interpersonal Reactivity Index To Measure PD, Trait-Emotional Intelligence Questionnaire for Emotion Regulation, and the Five Factor Personality Inventory (IPIP-50) for Stability. Machine Learning Via K-Means Cluster Analysis Determined Subgroup Profiles. Pearson Correlations Established Relational Strength of PD, Emotion Regulation and Stability Via IBM® SPSS ${ }^{\circledR}$ 24.0. This Research Approved By the Institution's IRB.

RESULTS: PD (alpha $=0.7$ ) mean scores were significantly $(\mathrm{p}<0.001)$ correlated with emotion regulation $(\mathrm{r}=-0.3)$ and stability $(r=-0.3)$, which were significantly correlated $(r=0.5)$ to each other. Cluster analysis converged on four significant clusters after nine iterations. Two clusters (45\%) aligned with correlation statistics with PD inversely related to emotion regulation and stability. Two clusters (55\%) yielded a direct relation between $\mathrm{PD}$, emotion regulation, and stability.

CONCLUSIONS: Machine learning via cluster analysis resolved conflicting reports of the moderate relationship of medical student PD, emotion regulation, and disposition. Some students reported PD levels in accordance to correlation analysis indicating empathy increased with emotion regulation and stable personality. Surprisingly, other students decreased PD under the same conditions, which was not revealed in correlation analysis, but the moderating factor has not yet emerged.

\section{ANALYZING THE IMPACT OF PERSONALITY ON AUTONOMY AND THE MEDIATOR ROLE OF MOTIVATION}

Robert Treat, PhD I Molly Falk-Steinmetz, MS I Cheryl Crawford, MS I Kristina Kaljo, PhD

Medical College of Wisconsin

PURPOSE: Autonomy is the ability to take charge of one's own learning. Students demonstrate autonomy within medical school when they set goals and identify resources to meet their learning needs, use the appropriate strategies to achieve them, and evaluate their learning outcomes. Intrinsic learner characteristics such as personality are linked to autonomy which is problematic when students have insufficient dispositional levels. Identifying a quality such as motivation that will mediate the adverse effects of personal traits can help learners achieve greater autonomy. The purpose of this study is to analyze the tri-variate relationship of medical student personality on autonomy as mediated by motivation. 
METHODS: In 2019-20, forty-one M1-M3 medical students who voluntarily completed the Index of Autonomous Functioning (scale: $1=$ not at all true $/ 5=$ completely true); Academic Motivation Scale (scale: $1=$ doesn't correspond $/ 5=$ corresponds exactly); Five-Factor Personality Inventory (IPIP-120, scale: $1=$ very inaccurate, $5=$ very accurate). Pearson correlations and stepwise multivariate linear regression were used to predict autonomy scores from conscientiousness (personality) scores and establish mediation via motivation. Inter-item reliability determined with Cronbach alpha. IBM® SPSS ${ }^{\circledR} 24.0$ was used for statistical analysis.

RESULTS: The empirical range of autonomy (alpha $=0.80$ ) scores was 41-68 with a mean $(\mathrm{sd})=57.3(6.2)$ and was significantly $(\mathrm{p}<.001)$ above the instrument's midline score $=45$. Statistically significant $(p<0.050)$ correlations were reported between conscientiousness and autonomy $(r=0.5)$ and motivation $(r=0.4)$. The correlation between autonomy and motivation was $r=0.4$. Linear regression of autonomy as the outcome variable and conscientiousness facets of self-efficacy (beta $=0.7$ ), dutifulness (beta $=0.5$ ), and orderliness (beta $=0.4$ ) as predictors generated a significant model $\left(\mathrm{R}^{2}=0.66, \mathrm{p}<.001\right)$. Introducing extrinsic motivation in this regression model mediated orderliness. CONCLUSIONS: Maintaining higher medical student autonomy scores by offsetting lower personality scores through extrinsic motivation is useful since personality traits are often fixed. Medical student autonomy is directly impacted by conscientiousness and motivation, and motivation mediated the impact of conscientiousness on autonomy.

\section{CAN FORMATIVE ASSESSMENTS ENHANCE STU- DENT ENGAGEMENT AND LEARNING IN A PAN- DEMIC ENVIRONMENT?}

\section{Cindy Funk}

\section{Burrell College of Osteopathic Medicine}

PURPOSE: The value of formative assessments in education is well-documented. Due to the pandemic, many medical curricula adapted a virtual delivery. In order to engage students in this environment, we sought to examine the effectiveness of guided formative assessments on medical student learning.

METHODS: During the COVID pandemic, the didactic curriculum for Burrell College of Osteopathic Medicine was delivered in a virtual-asynchronous format. In the Musculoskeletal I system, a series of online, formative quizzes was delivered via Learning-Catalytics for upper limb anatomy. Quizzes were composed of multiple choice, short-answer, matching, and identification questions, designed to provide active learning, feedback, and knowledge gap identification.
"In-session" quizzes were provided during virtual lectures, at 15-min intervals of lecture time targeting key concepts. "Post-session" quizzes were delivered after lectures to test higher order knowledge. Quizzes provided immediate, written feedback. ANOVA and correlation statistics were utilized to determine if quiz participation impacted summative exam performance.

RESULTS: There was robust usage of quizzes; students completed an average of 8/11 quizzes. To compare outcomes, students were divided into three groups: low engagement (completing 0-3 quizzes; 42 students), moderate engagement (completing 4-7 quizzes; 38 students), high engagement (completing $8-11$ quizzes; 92 students). ANOVA revealed significant differences amongst these groups on summative exam performance ( $f=9.41624)$. T-test revealed significant differences in summative exam performance between high $(84.8 \%)$ and low groups $(78.75 \%)$. Positive correlation between summative exam performance and number of quizzes completed was also found.

CONCLUSIONS: Adapting to virtual environments has been difficult for many medical students, creating isolation and lack of academic direction. We demonstrate positive engagement and feedback with formative assessments, indicating their value in engaging students, supporting learning and preparing for summative assessments.

\section{OSCE: OBJECTIVELY SCORING IN THE COVID ERA}

\section{Danh Le I Alisa Wray I Sara Stern-Nezer I Ariana Nelson}

University of California Irvine School of Medicine

PURPOSE: The Objective Structured Clinical Examination (OSCE) is a cornerstone of medical school assessment to ensure a high standard for clinical skills as students advance through curriculum. Due to the COVID pandemic, the University of California-Irvine School of Medicine adopted two different approaches to safely conduct medical student OSCEs.

METHODS: In approach 1, students were scheduled at the Clinical Skills Center (CSC) with mandatory masks and gloves. After a socially distanced orientation, each student entered their assigned room to interact with a tablet device connected with the standardized patient (SP) via a secured virtual platform. The standardized checklist was delivered via mail servers directly to each SP. By administering the examination in a recorded environment at the CSC, confidentiality of the case content and SP checklist was maintained. In approach 2, a 20 min entirely virtual encounter was utilized, consisting of a single case in which a silent faculty member (included for quality assurance) evaluated the student alongside the SP evaluator using identical checklists. 
After the encounter, students received five minutes of verbal feedback from both evaluators.

RESULTS: The history portion for both approaches was unchanged from prior, but the physical exam portion of each was challenging to standardize. The students were instructed to verbally explain the desired physical maneuver and rationale and SPs were instructed to give a verbal response. One exception was assessment of cranial nerves 2-10 where the SPs were instructed to perform the maneuvers in addition to verbally stating the results of the test. SPs were also instructed to request clarification of instructed physical exam maneuver as appropriate.

CONCLUSION: Learner assessment at a distance will likely continue in the COVID era. It is important to assess which OSCE formats are appropriate for standardized evaluation while comparing and contrasting methods in terms of safety and validity for quality assurance.

\section{EXPLORING STUDENTS' PERCEPTION OF ONLINE OSCE: A QUALITATIVE STUDY}

\author{
Maribel Aurelio | Samal Nauhria | Gimena Marzan | \\ Amitabha Basu
}

\section{St Matthews University School of Medicine}

PURPOSE: Since the start of the pandemic, we implemented virtual OSCE for the Preclinical students in the Basic Sciences. The purpose of the study is to know how students adapted to this new system and to understand the students' perceptions of their preparation, real-time experience during the OSCE process, challenges, and benefits of the virtual OSCE.

METHODS: This study used qualitative methods for data collection using semi-structured interviews of student focus groups. A total of 20 students, who took part in the virtual OSCE in this current year, were divided into 3 focus groups and interviewed. The online interviews were recorded with Olympus Voice Recorder and were transcribed in full. Lineby-line open coding was initiated to develop preliminary themes and subcategories. Later, axial coding was applied to group codes appropriately to gain knowledge around the aims of the study.

RESULTS: Four main themes were identified: Theme 1: PreOSCE preparation; Subcategories: Reading course materials; watching course videos; practicing with non-medicos (family members, peers) as standardized patients. Theme 2: Perceived fears and anxieties; Subcategories: Fear of technological failure; inadequate preparation; fearing judgmental standardized patients; fear of looking bad (academically) to the preceptor. Theme 3: Challenges faced during the virtual OSCE; Subcategories: Creating proper exam environment at home; performing satisfactory history-taking (difficulties in communication, such as eye contact, language barriers); performing satisfactory physical examination (lack of handson clinical practice, difficulties in verbalizing PE techniques). Theme 4: Students' perceived benefits of a virtual OSCE; Subcategories: Comfort of the home environment (less examroom awareness); not performing in front (in-person) of the standardized patient; absence of peer pressure; less insecurity about physical appearance; more understanding faculty. CONCLUSION: Students gained an understanding of how telemedicine works. Students should be provided with minimum IT requirements for a smooth online OSCE experience, which would alleviate anxiety. Students need to be trained beforehand on how to narrate physical examination techniques.

\section{DETERMINING THE RELATIONSHIP BETWEEN GAP YEARS, FIRST YEAR MEDICAL SCHOOL PER- FORMANCE, AND ACADEMIC BURNOUT}

Abdulai Bangura, MS. I Matthew Barvo, MS. I Iuliia Zhuravlova, MD, PhD. I Bernadette Scott, MD.

Trinity School of Medicine-Research Department

PURPOSE: There is a high prevalence of burnout among medical students. Students who experience burnout are more likely to have lower academic performance. Could student gap year(s) participation help reduce student burnout or increase academic performance at the medical school level? METHODS: Second-year medical students participated in a two-part survey. The primary survey will require general information disclosure including gap year(s) participation, pre-health experiences, and medical school course track. The secondary survey will consist of the Maslach Burnout Inventory-Student Survey (MBI-SS). Student G.P.As will be de-identified. Student G.P.As and Burnout scores will be categorized depending on the students total amount of gap years: $0,1-2$, and $3+$ years. The responses were analyzed with SPSS using ANOVA statistical testing. The study was approved by the Institutional review board of TMSU.

RESULTS: The study is currently in progress. Initial results from 48 responses demonstrate that medical students without gap year(s) had a mean GPA of 3.60, while those students with gap year(s) had a mean GPA of $3.28(\mathrm{P}=0.045)$. There were no correlations found between gap years and any component of the MBI-SS, e.g. exhaustion $(r=0.103$, $\mathrm{P}=0.593$ ). Additional statistical analysis will be completed once the limit of participants is reached.

CONCLUSION: Medical students who decide to not defer their education were found to have higher academic performance than medical students who took gap year(s) between college and medical school. Also, gap years were not found to help reduce the risk of student burnout. With 
more respondents, the statistical power of the findings will increase and be more representative of the medical student population. Further analysis will categorize the length of gap years.

\section{THE IMPACT OF GENDER ON RESIDENT EVALU- ATIONS OF FACULTY PERFORMANCE}

\author{
Allison Beaulieu, MD | Jennifer Mitzman, MD | Katherine \\ M. Hunold, MD I Simiao Li-Sauerwine, MD
}

The Ohio State University Wexner Medical Center, Columbus, OH, 43210 USA I The Ohio State University College of Medicine, Columbus, OH, 43210 USA

PURPOSE: There is a significant gender gap in academic medicine. Implicit bias impacts evaluations of female physicians in residency training and continues to influence factors pertaining to advancement as academic faculty. The goal of this study is to determine if faculty gender impacts evaluations of academic faculty by residents within the specialty of emergency medicine.

METHODS: A mixed-methods analysis will be employed to examine 14,669 teaching evaluations of faculty by residents at a single academic center in the years 2017-2020. Anonymized ratings of male and female faculty on a fivepoint Likert scale will be compared using the Student's T-test and chi-square test. Qualitative analysis of free text will be analyzed using grounded theory to examine narrative evaluations for gendered language. This study was approved by the IRB at The Ohio State Wexner Medical Center.

RESULTS: We expect to report a quantitative comparison between evaluations of faculty with respect to gender. We plan to perform subset analyses based on academic rank. We anticipate reporting qualitative outcomes in the form of major themes which emerge during analysis as well as a comparison of narrative evaluations between male and female faculty. Based on prior literature on gender differences and their impact on teaching evaluations, we expect to find gender differences in quantitative ratings of faculty as well as differences in qualitative analysis with respect to learner expectations and cited areas of strength for faculty. CONCLUSION: Implicit gender bias has previously been determined to impact evaluations of faculty. These disparities negatively impact promotion and tenure for female faculty. If there is found to be gender bias in the assessment of academic female faculty, evaluator training to mitigate implicit gender bias can be pursued to close the gender gap in academic emergency medicine.

HOW TO MEASURE "FIT": STANDARDIZED COMPARISON OF PROGRAM AND APPLICANT ALIGNMENT OF VALUES AND PRIORITIES
Kelly Dore, Jill Derby, Nimo Jama, Heather Davidson, Harold Reiter

McMaster University, Hamilton,Ontario, Canada; Altus Assessments, Toronto, Ontario, Canada

PURPOSE: NRMP surveys of programs and applicants identified "fit" as an important selection factor. However, little consensus exists on what "fit" is or its influencing factors. This study examines factors in applicant/program alignment in GME selection through perspectives of key SMEs.

METHODS: A Delphi survey was conducted in 2 rounds, consisting of 47 factors. Diverse SMEs participated in the Delphi and ranked factors on a Likert-type 4 point scale.

RESULTS: 38 SMEs responded in the survey's 1st round, 34 of 47 factors reached consensus with a 55\% consensus threshold. 4 additional factors reached consensus in the 2nd round. After integrating SME feedback, 30 factors remained. Factors were organized into 3 themes: culture, pedagogy and work environment.

CONCLUSION: Survey results will be used in a pairedcomparison tool for GME selection in Fall 2020 across multiple programs. This standardized method of evaluating "fit" provides insight at the time of GME selection.

\section{IMPLICATIONS OF USING AN SJT IN ADMISSIONS FOR PREDICTING FUTURE PROFESSIONALISM ISSUES}

Anurag Saxena, Kelly Dore, Harold Reiter, Loni Desanghere

University of Saskatchewan, Saskatoon, Saskatchewan, Canada; McMaster University, Hamilton, Ontario, Canada; Altus Assessments, Toronto, Ontario, Canada

PURPOSE: Admissions challenges include predicting student professionalism using traditional non-academic metrics. A PGME program piloted the use of the SJT, Casper, to measure non-academic qualities for selection.

METHODS: This study compared institutional impact of resident performance before and after SJT implementation. PGY-1 cohorts before $(2015 / 2014: \mathrm{n}=234)$ and after $(2017 / 2018 \mathrm{n}=237)$ SJT implementation were analyzed to compare professionalism, remediation, and associated costs. RESULTS: Control trainees had 12 professionalism concerns, 5 interventions and 5 remediations with 7 trainees with low non-MK ratings. CASPer-assessed trainees included 3 professionalism concerns, 1 intervention and 2 remediations with 3 trainees with low non-MK ratings. Total cost savings post-SJT implementation was $\$ 119,754.72$ CAD.

CONCLUSION: Results support including Casper to measure professional attributes in addition to existing metrics as an indicator of in-program professionalism. 
TOWARD THE DEVELOPMENT AND CONSTRUCT VALIDITY OF THE 7PS INVENTORY OF SELFREGULATED LEARNING TO IDENTIFY STUDENT ACADEMIC SUCCESS

Daria Ellis, Priyadarshini Dattathreya, Maureen

Hall Ross University School of Medicine, St. Michael, Barbados

PURPOSE: Self-regulated learning has been identified as a key factor that determines academic success. We used the principles of self-regulated learning and developed the 7Ps Inventory of metacognitive strategies that break down the task of 'learning' into strategies include planning and organizing, self-monitoring and evaluating academic progress through self-reflection. To ensure content validity, the item pool was evaluated by a panel of subject matter experts and students. The purpose of this study is to establish the construct validity of the 7Ps Inventory.

METHODS: We conducted an initial psychometric validation of the 7Ps Inventory of 36 items with 500 medical students. Exploratory (EFA) and confirmatory factor analyses (CFA) were conducted to assess the latent structure of 7Ps Inventory. Findings highlighted areas where the 7Ps Inventory required revision. Following revision, we conducted another psychometric validation with an additional sample of 191 first year medical students. We used EFA and CFA to assess construct validity and we assessed reliability using Cronbach's measure of internal consistency.

RESULTS: The final version of the 7Ps Inventory comprised 26 items (rated on a five-point response scale) that correspond to seven discreet components: Plan, Prepare, Participate, Process, Practice, Performance and Pause. The models for the final revised scales had good fit and the internal reliability of these scales was marginal to excellent, with Cronbach's $\alpha$ ranging from 0.52 to 0.86 .

CONCLUSION: Our preliminary evidence suggests that the 7Ps Inventory to help students reflect on their learning and assess their use of specific learning strategies is a psychometrically robust tool and will be used to identify areas where students need additional support resources.

COMPARING ONLINE AND IN-PERSON EDUCATIONAL WORKSHOPS FOR CANADIAN OCCUPATIONAL THERAPISTS: EXPLORING THE LEARNING EXPERIENCE

Sung Ha Kim I Ilana Bayer I Rebecca Gewurtz I Nadine Larivière | Lori Letts
School of Rehabilitation Science, McMaster University I Department of Pathology and Molecular Medicine, McMaster University I Department of Medicine, University of Sherbrooke

PURPOSE: The Do-Live-Well (DLW) framework is a health promotion approach that many occupational therapists (OTs) are interested in learning about. Although online education has become increasingly popular among health care professionals, studies of its effectiveness and learners' experience have been limited in occupational therapy education. The objectives of this study were to compare the effectiveness of the online and in-person DLW workshop for Canadian OTs and to explore participant experiences in both types of workshops.

METHODS: An explanatory sequential mixed-methods study design was used. In the quantitative phase, descriptive and inferential statistics were used to compare the effectiveness of the two educational methods at three points (pre, post, and 6-month follow-up). The primary outcome was knowledge change, and the secondary outcomes were changes in factors influencing the use of DLW in practice, satisfaction with the workshops, and the actual use of DLW. In the qualitative phase, an interpretative description methodology was used. Semi-structured one-on-one interviews conducted at follow-up were transcribed and analyzed using a six-step analysis process.

RESULTS: There were no statistically significant differences between groups in knowledge changes at three time points $(\mathrm{p}>0.57-0.99)$. There were statistically significant differences between groups in factors influencing DLW adoption $(p>0.001)$ and satisfaction with the workshop $(p>0.0005)$ at the post-test. Five themes were identified in relation to learners' workshop experience: (1) synchronous in-person interaction, (2) flexibility in online learning, (3) ease of access to learning, (4) comfortable learning environment, and (5) relevance to practice and interest.

CONCLUSION: There were no statistically significant differences between the groups in most of the quantitative data, and participants identified each method's benefits and challenges. The findings indicate online learning can be as effective as in-person learning. However, combining both methods' positive aspects may improve learners' educational experiences.

DO GRADUATING US STUDENTS HAVE THE SKILLS TO PERFORM THE ASSOCIATION OF AMERICAN MEDICAL COLLEGES (AAMC) CORE ENTRUSTABLE PROFESSIONAL ACTIVITIES FOR ENTERING RESIDENCY (CORE EPAS): ANALYSIS OF THE NATIONAL AAMC 2019 GRADUATION QUESTIONNAIRE (GQ)

\section{Douglas Grbic}

AAMC 
PURPOSE: The AAMC Core EPAs describe 13 activities that medical graduates should be prepared to perform under indirect supervision. Using national AAMC 2019 Graduation Questionnaire (GQ) data, we investigated relationships between observation/feedback frequency and self-assessed skill to perform each EPA across specialties.

METHODS: Chi-square and ANOVA tested EPA-specific associations between frequency of observation with immediate feedback in the clinical workplace ("frequently" $=>5$ times; "not frequently" = "2-5 times", "once" and "never") and skill acquisition ("acquired" = "agree" and "strongly agree"; "not acquired" = "neutral", "disagree" and "strongly disagree" that "I have the skills to perform each EPA) among AAMC 2019 GQ respondents entering emergency medicine $[\mathrm{EM}]$, internal medicine, family medicine, psychiatry, obstetrics \& gynecology, surgery or pediatrics.

RESULTS: Among 9,853 GQ respondents in 7 specialties ( $66 \%$ of all 14,975 respondents with complete data), proportions of respondents who reported frequent ( $>5$ times) observation/feedback differed across EPAs, ranging from 8\% (795/9,853; EPA13: Report patient safety concerns) to $93 \%(9,130 / 9,853$; EPA6: Oral presentation). Frequent observation/feedback was associated with skill acquisition for every EPA (each $\mathrm{p}<.01$ ). Specialty was associated with observation/feedback frequency for EPA4: Enter \& discuss orders/prescriptions, EPA7: Form clinical questions/retrieve evidence, EPA8: patient handover, EPA10: initiate urgent/ emergent evaluation/management, EPA11: informed consent, EPA12: Procedures and EPA13 (each $\mathrm{p}<.01$ ). Students entering EM were over-represented among those reporting frequent observation/feedback for EPA10-EPA13 and also among those reporting skill acquisition in 7 of 13 EPAs (4, $7,8,10,11,12$, and 13 ; each $\mathrm{p}<.01$ ).

CONCLUSIONS: There was wide variation across the 13 EPAs in observation/feedback frequency, which was associated with skill-acquisition endorsement across all EPAs. Specialty-specific differences suggest that EM curricular experiences may offer particularly robust opportunities for student observation/feedback in the clinical workplace in numerous EPAs; there may also be specialty-specific differences in student behaviors in seeking opportunities to perform various EPAs and requesting feedback.

\section{MEDICAL STUDENTS' PERCEPTIONS ON CHANG- ING OSTEOPATHIC MANIPULATIVE MEDICINE LAB PRACTICAL ASSESSMENT STYLES}

Yen-Chung Wang

VCOM-Auburn

PURPOSE: With the development of Osteopathic Core Competencies and Core Entrustable Professional Activities
(EPAs), there has been a shift towards competency-based curriculum in osteopathic medical education. Medical students at the Edward Via College of Osteopathic MedicineAuburn campus (VCOM-Auburn) utilize the traditional, randomized Osteopathic Manipulative Medicine (OMM) practical testing style, and they were surveyed on their preferences towards an OMM practical assessment modality to determine how receptive students would be to curricular change. The study evaluated the learning and assessment preferences of first- and second-year osteopathic medical students (OMS I \& OMS II) at VCOM-Auburn.

METHODS: Participants, regardless of sex, age, race, or academic achievement, were recruited using class announcement and emails. A 6-question anonymous and voluntary survey was conducted via iClicker to evaluate perception and readiness for change in OMM curriculum and assessment formats.

RESULTS: Out of the 308 enrolled first- and second-year students, 243 responded (78.9\%). Study results found that OMS I and OMS II students selected similar choices for each question, and most students preferred the current traditional OMM practical testing style over competency-based testing. However, there is a significant difference in the proportion of student satisfaction and testing preference between OMS I and OMS II classes; satisfaction with the current practical setup decreased from $82 \%$ among OMS I's to $68 \%$ among OMS II's, $\times 2(1)=5.114, \mathrm{p}=0.024$.

CONCLUSIONS: Data suggests that OMS I and OMS II at VCOM-Auburn are satisfied with the current traditional practical assessment with a significant decrease with the increase in seniority. Previous experience in traditional assessment may be a factor. The preference to utilize competency-based learning as medical education progresses predicts that students and residents with increased medical education experience acknowledge the importance of a more interactive and flexible curriculum. Therefore, this a is relevant consideration with the changes to the Single Accreditation System for Graduate Medical Education.

\section{EFFECTS OF ORGAN-SYSTEM COURSES OF THE FIRST TWO YEARS OF MEDICAL SCHOOL ON PERFORMANCE OF COMLEX-USA LEVEL 2}

\section{Kevin McNeil I Han Wang I QIng Zhong}

Rocky Vista University I Shenzhen DJI Sciences and Technologies Ltd, Shenzhen, China (Statistician)

BACKGROUND: Comprehensive Osteopathic Medical Licensing Examination of the United States (COMLEXUSA) Level 2-Cognitive Evaluation (COMLEX-USA Level 2-CE) is a board examination that every medical student in an osteopathic medical school must pass to graduate. 
Students usually take it in the third or fourth year of medical school. A few researchers have investigated the relationship between performance in preclinical/clinical sciences and performance on COMLEX-USA-Level 2-CE, but there is no study on the influence of each organ system course during the preclinical years on COMLEX-Level 2-CE performance. OBJECTIVES: We aimed to investigate the relationship between each organ system course and performance on COMLEX Level 2-CE. Our findings will help students focus on important basic sciences much earlier before preparing for COMLEX-USA Level 2-CE.

METHODS: Academic data from students matriculated at Rocky Vista University College of Osteopathic Medicine from 2011 to 2017 were obtained. Data included preadmission MCAT scores, course grades in the first two years of medical school, first attempt COMLEX Level 1 scores, and COMLEX-USA Level 2-CE scores. Pearson correlation coefficient, multiple linear regression, and backward stepwise regression were run with Sigma Plot 14 software. The highest correlation with COMLEX-USA Level 2 is the score on COMLEX Level 1; the next highest are third-semester Cardiovascular System (CVII) and Renal System II (RENII) courses. Multiple linear regression shows that only the average score in all year- 2 courses is a significant predictor of performance on COMLEX-USA Level 2; the average score in all year-1 courses is not significant. Backward stepwise regression shows that MCAT scores, third-semester CVII, RENII, Respiratory System (RSII), Principles of Clinical Medicine III (PCMIII), and fourth-semester Neuroscience System II (NSII) courses are significant predictors.

CONCLUSIONS: Performances in third-semester courses are the most important predictors of scores on COMLEXUSA Level 2-CE.

\section{EARLY PREDICTION OF THE RISK OF SCORING LOWER THAN 500 ON THE COMLEX 1: A STUDY OF PRE-MATRICULATION MCAT SCORES AND PRE-CLINICAL GRADES AT AN AMERICAN OSTE- OPATHIC MEDICAL SCHOOL}

Qing Zhong I Han Wang I Payton Christensen I Kevin McNeil I Matthew Linton I Mark Payton

Rocky Vista University I Data Scientist, Shenzhen DJI Sciences and Technologies Ltd, Shenzhen, China

BACKGROUND: The Comprehensive Osteopathic Medical Licensing Examination of the United States Level 1 (COMLEX 1) is important for medical students to be able to participate in clinical rotation and graduation. No predictive model is currently available to predict the outcome of the COMLEX 1 using our two-pass organ system curriculum.
OBJECTIVES: Our goal is to produce a reliable predictive model that will identify students who are at risk of scoring lower than 500 on COMLEX 1 at the earliest possible time. METHODS: Academic data from medical students who matriculated at Rocky Vista University College of Osteopathic Medicine between 2011 and 2017 were obtained. Odds ratios were used to assess the predictors for scoring lower than 500 on COMLEX 1. The predictive models were developed by either backward logistic regression or logistic regression and were based on performances on the Medical College Admissions Test (MCAT) before admission, as well as students' performances in preclinical courses during the first three semesters. The models were generated in about $82 \%$ of the student performance data and were then validated in the remaining $18 \%$ of the data.

RESULTS: Odds ratios showed that MCAT scores and final grades in each course of the first three semesters were significant in predicting a score lower than 500 on COMLEX 1. Performances on third-semester courses of Renal System II, Cardiovascular System II, and Respiratory System II were most important in prediction. Two predictive models had sensitivities of $65.8 \%-71 \%$, and specificities of $83.2 \%-88.2 \%$ in predicting a score lower than 500 on COMLEX 1.

CONCLUSIONS: Lower MCAT scores and lower grades in the first three semesters of medical school predict scoring lower than 500 on COMLEX 1.

\section{INFLUENCE OF MCAT RETESTING ON PER- FORMANCE OF PRECLINICAL MEDICINE AND COMLEX-USA LEVEL-1 AND LEVEL 2-CE}

Anton Nguyen Pham I Qing Zhong

Rocky Vista University

BACKGROUND: The Medical College Admission Test (MCAT) is utilized as one of the preadmission variables by the medical school admissions committees in the selection of students since 1928 in United States. Students are permitted to retake the MCAT up to three times in one calendar year and four times across two calendar years, with a maximum of seven attempts in their lifetime in order to maximize their score. The MCAT score is used as a predictor of how well a student can perform in medical school, with extensive research investigating the relationship between MCAT scores and preclinical performance as well as medical board examinations, yet sparse research have focused on the effects of retaking the MCAT. Furthermore, there has been no exploration into the influence of retesters' MCAT scores and the number of MCAT attempts on COMLEX Level 1 and Level 2-CE in literature. Objectives: Our goal was to investigate whether MCAT retaking affects the performance of preclinical courses and board examinations. 
METHODS: Data from 904 students who matriculated at Rocky Vista University College of Osteopathic Medicine during 2012-2017 included MCAT scores on the first attempt, second attempt, third attempt, fourth attempt, preclinical course scores, and first attempted scores of COMLEX Level 1 and Level 2 CE. One-way ANOVA, X2 test, and Pearson correlation coefficient were performed. Objectives: The analysis revealed that compared to nonretesters, retesters had significantly lower first-time and average MCAT scores, with the lowest seen in those who retook it four times. In addition, scores of COMLEX Level 1 in retesters who took the MCAT four times were significantly decreased compared to that in non-retesters.

CONCLUSION: Increased attempts of the MCAT negatively influenced the performance of COMLEX Level 1 and Level 2-CE.

\section{THE ROLE OF EXAMINATION RANKINGS IN MEDICAL STUDENTS' EXPERIENCES OF THE IMPOSTOR PHENOMENON}

\section{Thomas Franchi I Nigel Russell-Sewell}

The Medical School, The University of Sheffield I Department of Biomedical Science, The University of Sheffield

PURPOSE: First coined by Clance and Imes in 1978, the term 'impostor phenomenon' is "used to designate an internal experience of intellectual phonies". People who experience this have deep thoughts of fraudulence regarding their professional or intellectual activities. This perception of illegitimacy leads sufferers to attribute their success to error, preventing high achievers from recognising their successes and inhibiting development in self-esteem.

METHODS: This research aimed to better understand the relationship between medical students and the impostor phenomenon, in the context of examination rankings. An ethics-approved action research project was undertaken at The University of Sheffield, using a pragmatic approach to integrate quantitative and qualitative data via a questionnaire, focus groups and interviews. The key quantitative measure used a validated instrument, the Clance Impostor Phenomenon Scale (CIPS), which uses twenty items to give scores between $20-100$.

RESULTS: Altogether, 191 questionnaire responses were recorded, with 19 students attending a focus group or interview. The cohort's mean CIPS score was $65.81 \pm 13.72$, signifying that the average student had "frequent" impostor experiences. Notably, females scored 9.15 points higher than males on average $(\mathrm{p}<0.0001)$. Examination rankings were very frequently cited as a major contributing factor to students' impostor feelings and linear regression revealed an increase of 1.12 CIPS points per decile that a student dropped down the examination rankings $(\mathrm{p}<0.05)$. Further, ranking in the bottom $10 \%$ of the cohort led to a 6.08-point increase on average $(p<0.05)$. Students' quotes underscore the data presented and offer an authentic insight into their experiences.

CONCLUSIONS: Based on this research, four specific recommendations for practice were formulated, which are intended to provide medical schools with opportunities for pedagogical evolution and innovation. When implemented, these recommendations will improve the educational experiences for medical students who experience the impostor phenomenon, specifically with a view to mitigating the impact of examination rankings on these experiences.

\section{DO SCHOOL-BASED USMLE TESTING CENTERS PROVIDE A HOME-FIELD ADVANTAGE?}

Pamela O'Callaghan I Kelly McCarthy I Emily Pernas

\section{USF Health}

PURPOSE: The onset of the COVID-19 pandemic forced commercial testing centers to close worldwide, leaving medical learners unable to complete their USMLE Step exams. In response, six U.S. medical schools were selected to create secure spaces and train staff to administer medical licensure exams. This abstract describes examinee feedback from learners who took their USMLE Step exam at the University of South Florida (USF) regional testing center.

METHODS: A four-question survey was sent to 175 examinees that tested at the USF regional testing site. Questions evaluate the following objectives: whether the testing center and staff fit the examinees' expectations, whether the option to test at the USF center lowered examinee stress, and whether examinees' perceptions utilized the USF test center improved their performance. Response options included yes, no, or neutral. Chi-squared test of independence assessed differences in survey responses $(\mathrm{p}<.01)$. Additionally, examinees could provide narrative feedback.

RESULTS: The survey was completed by 130 examinees (74\% completion rate). Responses were overwhelmingly positive, with $95 \%$ of respondents reporting that the test center met their prior expectations and that the option to test at USF made the testing process less stressful. The testing center staff met examinee expectations, with $98 \%$ responding positively. Perceptions that testing at the USF center helped increase an examinee's score were reported more often by USF medical students than by medical students from other institutions. There was a significant relationship between the two variables, $\mathrm{X} 2(2, \mathrm{~N}=130)=14.9, \mathrm{p}<.01$.

CONCLUSION: Based on the survey responses, various operational factors contributed to a positive student testing experience. Outcomes, including narrative feedback, are of 
interest to multiple stake-holders. This project is beneficial to medical students preparing for their licensure exams, commercial testing centers, and the National Board.

\section{USING DISCRIMINANT ANALYSIS TO ASSESS THE VALIDITY OF A PREDICTIVE REGRESSION MODEL FOR IDENTIFYING STUDENTS AT RISK OF FAILING USMLE STEP 1}

\author{
Andrea Vallevand I Brooke Shipley I Yenya Hu \\ Wake Forest School of Medicine
}

PURPOSE: The accurate detection of students at academic risk can permit the deployment of interventions, such as the deliberate use of question banks, to identify and patch knowledge gaps. A priori detection is particularly critical for licensing examinations, where failure may impact residency aspirations. The current research explores the validity of a regression model employed to predict the risk of Step 1 failure.

METHODS: Preclinical Customized Assessment System examination and Step 1 scores were collected from three cohorts. Regression analysis was conducted and a roster of predicted Step 1 scores calculated for the subsequent cohort. The USMLE passing score and documented standard error of measurement (194 and \pm 6 , respectively) informed an "at risk range". Students identified "at risk" were offered additional academic coaching during the dedicated Step 1 study period. Discriminant analysis, employed retrospectively, investigated Step 1 pass/fail results and the accuracy of the regression analysis. RESULTS: The regression analysis predicted scores, categorized as "at risk", for 8/127 (6.3\%) students. Predicted scores ranged between 186 and 203. Of these eight students, four took academic leave, three failed Step 1 and one passed. Among the 117 students who took Step 1, during the designated examination cycle, five of them $(4.3 \%)$ failed. The discriminant analysis accurately predicted the five students that failed. Three of these students were flagged as Step 1 risks initially, by the regression analysis, with their respective predicted scores ranging between 186 and 199.

CONCLUSION: Regression analysis has provided our academic coaching program with a frame of reference for where along the pass/fail continuum students are located at the start of the dedicated Step 1 study period. Discriminant analysis is used to retrospectively validate these initial predictions, particularly when students do not engage in academic coaching.

\section{EFFECT OF COVID-19 PANDEMIC ON COMLEX LEVEL 1 PERFORMANCE}

Sarah A. McCarthy | Jack Lee | Christine Kell

Lake Erie College of Osteopathic Medicine
PURPOSE: Due to the COVID-19 pandemic, many medical students postponed taking their COMLEX LEVEL 1 board exams in 2020. This was due to quarantine-related delays and closures at testing sites. Typically, LECOM students take the COMLEX Level 1 examination in May or early June of their second year; however in 2020, the testing site closures and scheduling issues caused most students to delay their exam.

METHODS: To determine whether exam site closures impacted Level 1 scores of LECOM Erie students, we analyzed Level 1 scores from 2020 and 2019. We hypothesize that the delay in test dates for the NBOME COMLEX Level 1 exam is correlated with a reduction in mean score. RESULTS: We observed the following changes of the percent of students taking their Level 1 exams in May-July comparing 2019 to 2020 : $58.1 \%$ vs $26.5 \%$ (May), $30.2 \%$ vs $45.2 \%$ (June), and $7.7 \%$ vs $23.9 \%$ (July), respectively. Additionally, we analyzed the changes in average scores in May-July comparing 2019 to 2020: 591.1 vs. 605.0 (May), 527.2 vs 546.1 (June), and 521.9 vs. 529.4 (July). The average score on COMLEX Level 1 was 562 in 2019 vs 554 in 2020.

CONCLUSIONS: The average monthly score on COMLEX Level 1 was consistently higher than in 2020; however, the overall mean average score was lower for students testing in 2020 as compared to 2019. We attribute this to students delaying their test date to later months. Further investigation is warranted to determine whether this was due to examination site closures or study habit changes due to the global COVID-19 pandemic.

\section{IDENTIFYING MASTER DEGREE PREDICTORS OF MEDICAL SCHOOL SUCCESS IN THE PRE- CLERKSHIP CURRICULUM}

Jennifer Fischer I Katharine Milani I Shiyuan Wang

Rowan University School of Osteopathic Medicine

The purpose of this study is to retrospectively look at the relationship between Graduate School of Biomedical Science (GSBS) performance and pre-clerkship medical school performance to identify performance metrics that may predict success.

METHODS: RowanSOM students who matriculated in the past five years $(\mathrm{N}=46)$ with previous masters coursework from RowanGSBS were used in this study. Successful students were defined as those who never failed a course or COMLEX Level $1(\mathrm{~N}=26)$ and struggling students were defined as those who failed at least one course or Level 1 $(\mathrm{N}=20)$. To compare the metrics of these two groups, a series of T-tests were applied. ANOVA analyses and post hoc tests were then used to compare performance metrics 
among successful students, students who had one failure, and those who had multiple failures. Lastly, Pearson's correlation analysis examines the associations between GSBS course performance and COMLEX Level 1 performance.

RESULTS: Between the two groups, struggling students showed statistically lower GPAs in biochemistry $(\mathrm{t}=-2.253$, $\mathrm{p}=0.03)$, microbiology $(\mathrm{t}=-2.574, \mathrm{p}=0.02)$, biomedical anatomy $(\mathrm{t}=-2.797, \mathrm{p}=0.02)$ and immunology $(\mathrm{t}=-8.54$, $\mathrm{p}<0.01)$ courses. Furthermore, ANOVA and post hoc tests indicated that the performance difference in these four GSBS courses was between successful students and those that had multiple failures. The correlation analyses also revealed statistically significant and moderate correlations between COMLEX Level 1 score and three performance metrics: overall GSBS GPA $(r=0.40)$, microbiology $(r=0.047)$, and exercise physiology $(\mathrm{r}=0.49)$ grades.

CONCLUSION: Results will be used to guide curriculum decisions in GSBS and advise students on what minimum overall GPA is beneficial for students' preparation for medical school. It is hoped that this analyses can be used as an example for other schools to better examine their admission pool, to advise the admissions committee on the potential of successful candidates, and to better support struggling students within each school's demographic with targeted curriculum.

\section{AFFIRMING INSTITUTIONAL ASSESSMENT EQUITY WITH DIFFERENTIAL ITEM FUNCTION- ING (DIF)}

\section{Brock Mutcheson I Richard Vari}

\section{Virginia Tech Carilion School of Medicine}

PURPOSE: Virginia Tech Carilion School of Medicine (VTCSOM) institutionally developed assessments are regularly and rigorously investigated before individual student performances are interpreted for pass/fail decisions. Higherstakes summative assessments require higher degrees of validity evidence than formative assessments. In this analysis, we extend these efforts by implementing a psychometric approach to further affirm validity and equity in VTCSOM student assessments.

METHODS: We evaluated assessment data for differential item functioning (DIF) to identify the extent to which items might be measuring different abilities for members of separate subgroups. An item displayed potential DIF if people from different groups with the same underlying true abilities had different probabilities of a correct response. Items displaying potential DIF were flagged for further review by expert stakeholders. We compared two methods for detecting DIF: 1) Mantel-Haenszel Procedure, and 2) Item-Response Theory Approach.
RESULTS: The DIF approaches resulted in differences in the proportions of items falling into each of two mutually exclusive categories: i) non-DIF, and ii) potential DIF. Content experts and focal groups were recruited to informally review and discuss flagged items in order to determine whether the source of item differential performance was related to the construct being measured.

CONCLUSIONS: Differential Item Functioning (DIF) is an important empirical approach to increasing confidence in decisions based on performance on high-stakes assessments. However, the selected approach may have implications for potential DIF detection, which indicated that methodology transparency is important. The results also highlighted the need for the implementation of consistent quality stakeholder reviews of flagged assessment items. Institutions can apply DIF procedures to establish evidence of item equity and increase confidence in institutional assessments.

\section{CONVERGENT VALIDITY OF A REVISED TEAM- WORK ASSESSMENT TOOL}

\section{Kathryn Kerdolff}

\section{Louisiana State University Health Sciences Center}

PURPOSE: We have shortened our observer-based Teamwork Assessment Scales (TAS) for use in our IAMSE research grant to develop a quantitative measurement suite to assess teamwork in healthcare. We wanted to demonstrate convergent validity of this Quick TAS (Q-TAS) in a population of health professional students participating in simulation-based team training.

METHODS: The Q-TAS is a 5-item instrument using a 6 -point Likert type scale ( $1=$ definitely no to $6=$ definitely) divided into three subscales: team-based behaviors (TBB, two items), shared mental model (SMM, one item), and adaptive communication and response (ACR, two items). We compared this instrument to the TeamSTEPPS ${ }^{\text {TM }}$ Team Performance Observation Tool (T-POT), a 23 -item observerbased teamwork assessment tool using a 5-point Likert scale ( $1=$ very poor to $5=$ excellent) divided into 5 subscales: team structure (four items), communication (four items), leadership (five items), situation monitoring (five items), mutual support (four items). Two trained observers rated the team performance of an inter-professional student team performing two simulation-based OR scenarios divided by a debriefing focusing on team-based competences. We calculated descriptive statistics for each subscale of the tools. For each tool, we compared the pre- and post-debriefing team scores using t-tests. A p-value $<0.05$ was the significance threshold.

RESULTS: Statistically significant improvements in scores occurred for all three Q-TAS subscales $(\geq 0.9$ units for TBB 
$(\mathrm{N}=49), \mathrm{p}<0.001 ;>1$ unit for SMM $(\mathrm{N}=7), \mathrm{p}=0.047 \&$ ACR $(\mathrm{N}=7), \mathrm{p}=0.045)$ and for all five TPOT subscales $(0.8$ units for team structure $(\mathrm{N}=16$ to 18$), \mathrm{p}=0.03 ;>1$ unit for all other subscales $(\mathrm{N}=16$ to 18$), \mathrm{p}<0.001)$ from the first scenario performance to the second scenario performance. CONCLUSION: We demonstrated convergent validity for the Q-TAS and TPOT for a population of inter-professional students in simulation-based OR team training. We plan to use each of these observer-based tools to help validate our quantitative measurement suite.

\section{Curriculum}

\section{STOP THE BLEED AND START WORKING WITH INTERPROFESSIONAL HEALTH STUDENTS}

Amy Stickford Becker, MA I Barbara Duerst, RN, MS I Michelle Ostmoe, BS I Amanda DeVoss, MMS, PA-C I Lisa Steinkamp, PT, PhD, MBA I Jamie Hess, MD

\section{University of Wisconsin}

PURPOSE: Interprofessional education and preparedness are hot topics in health professional education. We implemented the national Stop the Bleed curriculum with an interprofessional group of first year students. During the activity, students were introduced to the roles and responsibilities, perspectives, values and expertise of one another; identified knowledge, skills and abilities that they bring to an emergency; and learned skills to help control bleeding in emergencies.

METHODS: Stop the Bleed is a national campaign to train, equip, and empower citizens to help in a traumatic bleeding emergency. In 2019, we implemented the campaign with 284 first year medical, physician assistant, public health, genetic counseling and physical therapy students during a 90-min session. Students were assigned to an interprofessional team of 8-10 students/table in two adjacent rooms. Forty volunteer physician assistants, nurses, community EMTs, residents, and physicians co-facilitated. Students participated in an introductory activity; the Stop the Bleed curriculum, which includes learning to apply direct pressure, packing wounds and applying tourniquets; and then a debrief.

RESULTS: 219 out of 284 students ( $77 \%$ response rate) completed a 9-question evaluation survey at the end of the session. Participants indicated the session was highly successful in achieving the intended outcomes, with $99 \%$ somewhat or strongly agreeing that they were satisfied with the session, $97 \%$ somewhat or strongly agreeing that the session was relevant to their area of study, and $85 \%$ somewhat or strongly agreeing that they benefitted from the interprofessional nature of the activity. Several students have followed up to become future facilitators for the Stop the Bleed campaign.

CONCLUSION: Implementing the Stop the Bleed curriculum as an interprofessional activity is an effective approach to teaching content and benefits both learners and facilitators as a valuable exercise in providing patient care in a collaborative team environment.

THINKING OUTSIDE THE SQUARE IN CULTIVATING INTERPROFESSIONAL PROFESSIONALISM IN OPTOMETRY

Kwang Meng Cham I Heather Gaunt

The University of Melbourne, Victoria, Australia

PURPOSE: Scholarship on teaching professionalism is limited in Optometry. There is no existing research focusing on the key conceptual content for an optometric curriculum. This project aims to provide inter-disciplinary learning and engagement for students in Optometry, Arts Management/ Curatorship and Animation. Focused on the physical environment of the Grainger Museum, students experienced object-based learning opportunities in group contexts, designed to promote personal and professional learning and reflection in the areas of ethical dilemmas, communication and collaboration across different disciplines.

METHODS: Optometry students experienced activities that focused on ethical dilemmas, including dealing with present or future 'moral distress' in health professional contexts. Arts Management/Curatorship students provided insights and ways of connecting to the selected objects, and Animation students assisted in the video production.

RESULTS: Together, $84 \%$ of the students $(n=70,100 \%$ response rate) reported that this task increased their understanding and awareness in professionalism and heath ethics. $86 \%$ believed that their communication and interpersonal skills will improve after this activity. $89 \%$ found group work more enjoyable compared to previous experiences.

CONCLUSIONS: The project has enhanced students' awareness in professionalism and ethical dilemmas. All students have an increased understanding and awareness of professionalism, teamwork, communication and collaboration across different professions and perspectives. This project has provided specific supported learning opportunities for students across disciplines to engage in unique ways. It has encouraged them to consider and appreciate the perspectives and skills of students from other disciplines, and how to communicate and work effectively in team-based settings.

LEADERSHIP FOR MEDICAL STUDENTS CURRICULUM DEVELOPMENT USING DESIGN THINKING AND STUDENT CO-CREATOR 
Alexa Lisevick, BS | Jennifer Dreiling, MD, FCAP | Rahul Anand, MD, MSc, MBA, FACP I Richard Feinn, MS, MA, $\mathrm{PhD}$

Frank H Netter MD School of Medicine, Quinnipiac University, North Haven CT

PURPOSE: Two faculty members and one second year medical student co-created a pilot leadership curriculum based on the principle that all physicians are leaders; our end goal was to empower medical students to lead themselves, teams, and impact systems.

METHODS: Utilizing student-driven needs assessment and curriculum design, the 16-week leadership course was uniquely developed to be centered on emotional intelligence and psychological safety. Eight pre-clinical medical students met weekly for 2-3-h long sessions throughout the semester for a total of 13 small group in-person sessions. Weekly session effectiveness surveys enabled rapid quality improvement in satisfaction and engagement. Students self-assessed their confidence to perform in multiple competencies using a 5-point Likert scale $(1=$ Strongly Disagree, $2=$ Disagree, $3=$ Neutral, $4=$ Agree, $5=$ Strongly Agree) within the 3 leadership domains (self, team, system) both prior to and at course conclusion; paired t-tests compared individual student's pre- and post-course responses. Qualitative closing reflections and student-led focus groups captured students' ability to apply what they learned to activities outside the course.

RESULTS: Pre- versus post-course, student self-evaluations demonstrated significantly improved confidence levels in competencies across all leadership domains: self, 3.61 to $4.39(\mathrm{p}=.001)$; team, 3.34 to $4.22(\mathrm{p}<.001)$; system, 2.80 to $3.70(\mathrm{p}=.003) .100 \%$ of students in the pilot $(\mathrm{n}=8)$ agreed that psychological safety within the learning environment contributed significantly to their understanding and overall experience. In qualitative reflections, all participants noted that a vital strength of the course was its direct relevance and immediate applicability to their ongoing medical school activities and roles, including student interest groups and team-based learning activities.

CONCLUSIONS: Our successful pilot curriculum demonstrated that leadership competencies can be taught and readily implemented by physicians-in-training. Involving students early and often results in curriculum that is highly relevant and a learning environment that fosters psychological safety.

\section{A CULINARY MEDICINE ELECTIVE COURSE INCORPORATING LIFESTYLE MEDICINE FOR MEDICAL STUDENTS}

Shinichi Asano I Amy E. Jasperse I Dina C. Schaper I Robert W. Foster I Brian N. Griffith

\section{WVSOM}

PURPOSE: The purpose of this study was to describe our culinary medicine elective course with a lifestyle modification focus and to evaluate the students' perceived knowledge, attitudes and self-efficacy in lifestyle medicine.

METHODS: Pre- and post-surveys including quantitative assessment, Likert-type questions, and one open-ended response question to assess students' perceived knowledge of nutrition and lifestyle medicine were distributed to osteopathic medical students who participated in the culinary medicine elective course. The Mann-Whitney U test and dependent $t$ test were used where appropriate based on normality.

RESULTS: Compared to the pre-course survey, students who responded "Strongly agree" in questions related to medical nutrition therapy in the post-course survey were 26.5 to $31.3 \%$ higher $(\mathrm{p}<0.05)$. Based on the post course survey $(\mathrm{n}=34), 33$ students responded either "Strongly agree" $(n=25,73.5 \%)$ or "agree" $(n=8,23.5 \%)$ to the question of "increased my knowledge of nutrition".

CONCLUSIONS: Culinary medicine course with a lifestyle medicine focus may be effective in increasing medical students' confidence and perceived knowledge of nutrition and lifestyle medicine.

\section{ORIGINAL RESEARCH BASED CURRICULUM IN A PHYSICIAN ASSISTANT PROGRAM}

\section{Shinu Kuriakose}

New York Institute of Technology

FOCUS: Physician Assistant students in the New York Tech program in New York, USA made a transition in their Research I-IV classes, over 4 semesters, to an original project-based deliverable regarding a topic that interested the students. The goal of this project was that after this original research study, the students could submit their work to a peer-reviewed journal after feedback from their faculty mentor. The vast majority of Physician Assistant programs in the world engage in a research project which is primarily literature review based. This experience familiarizes the NYT PA students with research protocols and processes. METHODS: The students in groups of 4 decide on a topic they are interested in and must then submit a few research articles, already published, in that domain as a guide. This 1 st research class culminates in a PowerPoint presentation 
and a proposal paper which are both graded. The students then fill out the IRB application, answer questions describing their research process, submit the survey methodology, and await approval before starting their research. Once data is obtained, the focus is on data analysis appropriate use of statistical software utilizing SPSS, exploring correlation, and causation. The final part is incorporating this study in a publishable format.

CONCLUSION: As this process is one year old, the conclusions of the project are still being awaited. Student feedback has been positive so far as this exposes students to the way a real publishable research process is conducted and this awareness will help them moving forward. There is also increased interest from the faculty perspective, as faculty often choose projects which mutually is beneficial for them and the students. It is the hope that the students become published authors within 6 months of their graduation and help them to move forward with clinical studies soon.

\section{EFFECTIVE PROCESS APPLIED TO NEW COURSE DEVELOPMENT}

\section{Mari Hopper}

Sam Houston State University College of Osteopathic Medicine

PURPOSE: With a recognized shortage of physicians, there has been impetus for development of new medical schools. Additionally, existing medical schools work to "refresh" curricula to align with adult learning theory and active engagement of students. Subsequently, faculty are called to generate new courses despite lack of training. To assist faculty at a new medical school, a process was developed and visual organizer created to guide faculty through new course development.

METHODS: Process for course development included three phases. 1) Course Director Preparation: course directors gathered materials including COMLEX-USA Blueprints, NBME content guides and reference textbooks, outlined course framework, topics, disciplines, number of sessions, course calendar, and identified membership for course development team. 2) Course Team Collaboration: Interdisciplinary teams conducted "huddle" meetings to sort topics into sequence, write and map objectives, determine course delivery methods, establish policies and grading, and establish syllabi elements. 3) Individual Faculty Authoring: Course directors invited discipline specific faculty to develop and deliver course sessions. Faculty wrote session objectives, developed deliverables, wrote exam items, submitted session mapping, and solicited peer evaluation. With staff assistance, the course director accepted, reviewed and prepared all materials for upload to the course management system, submitted textbook and room requests, and other tasks. A single paged visual illustration of the process was developed and distributed to all faculty.

RESULTS: Faculty report having the process for new course development available as a visual organizer improves efficiency and effectiveness as it directs efforts of interdisciplinary teams and allows quick onboarding of team members. CONCLUSION: Once the process for development of new courses was established, generating a visual organizer was an effective method of guiding faculty through the process. The process and organizer could easily be adapted for use in curricular development in a variety of medical and other health related programs.

\section{DIET QUALITY AMONG FIRST-YEAR OSTEO- PATHIC MEDICAL STUDENTS}

Sarah C Holzmann, MSc I Sara Goldgraben, MD, MPH, MBA I Emily A. Johnston, PhD, MPH, RDN, CDE

California Health Sciences University College of Osteopathic Medicine

PURPOSE: Nutrition is inconsistently taught in the training of medical students. We are integrating nutrition into a systems-based curriculum at a college of osteopathic medicine and teaching culinary medicine classes relevant to each system. We seek to measure the effects of this training on personal dietary choices.

METHODS: First year osteopathic medical students took an online 160-item validated food frequency questionnaire (FFQ) as a part of their designated nutrition content. They were provided with a link and a username and password and received a report with a Healthy Eating Index (HEI)-2015 score, which measures adherence to the Dietary Guidelines for Americans and is scored out of 100, upon completion. The report also included shortfall nutrients and foods to increase intake of to help them meet nutrient goals. These reports were integrated into the subsequent nutrition content with instructions on how to interpret the reports and how their dietary intake compared to the guidelines they were learning.

RESULTS: All ( $\mathrm{n}=80)$ first-year osteopathic medical students took part in the initial dietary assessment. The sample was $48 \%$ female with a mean age of 26 years of age (range: 21-42 years of age). The mean BMI was $24.6 \mathrm{~kg} / \mathrm{m} 2$ (healthy weight) and they reported low-moderate levels of physical activity. Overall HEI-2015 score was 67.3 (43.8-89.6) out of 100. Average sodium intake was $4,030 \mathrm{mg}$ and fiber intake was 23.8 g per day.

CONCLUSIONS: Medical student HEI-2015 score was higher than the national average, but sodium intake exceeds and fiber intake falls short of evidence-based guidelines. 
Comparing these data to a second administration of this FFQ at the end of the year will aide us in answering the question of whether an integrated nutrition curriculum, including hands-on culinary medicine classes, improves overall diet quality, measured by HEI-2015 score, among first year osteopathic medical students.

\section{THE INFLUENCE OF INSTITUTIONAL TRUST ON THE ACADEMIC DECISIONS OF MEDICAL STUDENTS}

Promise Bood I Hannah Howse I Patrick Rendón I Tom Markle

The University of New Mexico School of Medicine, Albuquerque, NM, 87131, USA

PURPOSE: Research regarding medical students' trust in their institution and willingness to follow the presented curriculum is currently lacking. Yet, the trust dynamics between students, their instructors and institution, may offer insight into the academic performance and overall clinical confidence of future physicians.

METHODS: An initial survey, distributed to pre-clinical medical students, was designed to gauge their trust in relation to board exam preparation and their curriculum, as well as the institution. Survey analysis prompted an exploration into several themes related to trust, that were then discussed in a focus group setting. Two classes of pre-clinical medical students have participated in the survey and focus group analysis.

RESULTS: Of the 101 survey responses, $49 \%$ of respondents indicated they were 'neutral' that the school was "adequately preparing [students] for STEP 1". Focus group discussions exposed strengths in the curriculum including 'instructor reliability' and 'organization'. These discussions also resulted in recommendations for interventions, including 'faculty acknowledgment of STEP 1' and 'consistency' in instruction methods in order to support a comfortable and trustworthy learning environment.

CONCLUSION: Based on available data, students perceive there to be a lack of harmony between the curriculum, as presented, and future board exams. Our initial recommendation is to have board pertinent information clearly outlined within the curriculum, while also emphasizing that certain topics may hold greater clinical importance. This research could provide invaluable information to medical educators concerning students' perception of best practices when creating and presenting material to optimize student reception and engagement with the curriculum. Our intent is to perform more data collecting cycles for each class as they progress through the medical curriculum.

\section{RESEARCH MENTORSHIP IN MEDICAL EDUCATION}

Alberto Muisto

Eastern Virginia Medical School

PURPOSE: Scientific training in medical education contributes to improve current scientific research and enabling better health outcomes. The goal is to present an educational approach that allows the students to contribute to a better understanding of medical neurosciences.

METHODS: Medical and undergraduate students selfselected themselves and submitted their resumes and a scientific research justification followed by a working research plan. All students performed a systematic review, formulated a scientific questions or hypothesis, and develop an experimental approach to test in the laboratory. Students interacted with laboratory personnel and received feedback about their progress. Assessments were based on: i) successful identification of scientific premises, description of the experimental designs, summary of results and alternative methods, impact of disease, presentation of results in an abstract, poster or manuscript; ii) laboratory techniques, presentation and writing skills, professionalism, and teamwork. A feedback was conducted before, during, and after to evaluate this mentorship as a learning experience.

RESULTS: 42 students which represent of $9 \%$ of the medical student's population per year, requested voluntarily to participate in this educational experience. They showed more availability during the second semester working in presence or online; $100 \%$ of the student completed a literature review; $35 \%$ of the students obtained certifications which allowed them to work with at least 2 or more laboratory techniques. $54 \%$ of students were able to be named as coauthors in scientific manuscripts, and $88 \%$ of the students were able to coauthor a presentation of poster. One student also received an award for his project in this mentorship program.

CONCLUSION: This optional educational activity contributed to improve the research environment in the medical curricula. This mentorship showed feasibility during the course of the medical school and it has been associated with greater satisfaction from the students with their training as well as greater opportunities for academic scholarly.

\section{DISCOVERY LEARNING PROGRAM: PROVIDING GLOBAL AND LOCAL LEARNING OPPORTUNI- TIES TO FIRST YEAR MEDICAL STUDENTS}

Olivia C. Coiado

University of Illinois at Urbana-Champaign 
PURPOSE: The Carle Illinois College of Medicine curriculum integrates basic and clinical sciences with engineering. At the end of the first year, students have an opportunity to engage in a clinical, research or global immersion through the Discovery Learning Program. The Discovery Learning course is up to 6-week elective designed to provide students with valuable qualitative and affective learning experiences, enhance their curiosity and creativity applying the inquirybased learning approach, develop technical competency and foster strong bonds of friendship and camaraderie among students and faculty.

METHODS: The course is divided in four categories: clinical, research, electives, and global immersion. Students can receive up to 6-credits after completion of the course. A total of 28 students with 35 projects were enrolled in the course during Summer of 2020: 16 students completed the research immersion, 8 students completed clinical immersion and 11 students completed electives. Students completed projects under supervision of a mentor: a clinical or a researcher faculty.

RESULTS: Some of the immersions include case report about challenges of diagnosing acute histoplasmosis and a rare case of eccrine porocarcinoma with metastic spread, research about cardiac pacing using ultrasonic pulses, data analysis, grant and manuscript writing and electives focused on COVID-19 literature review and a Medical Spanish course. After the immersion, students presented their experiences in a Pecha Kucha (storytelling format) event.

CONCLUSION: The main outcomes of the Discovery Learning Program were: 1) medical students reported they had a valuable qualitative learning experience which encouraged their deep thinking about the interaction between medical/engineering and its environment, 2) the experience exposed students to cultures and ways of life of a diverse groups of people, 3) students were very satisfied with the program. Students appreciated the professional experience and the possibility of the immersion in different environments to better understand health care problems.

\section{BUILDING STUDENTS' CULTURAL COMPETENCY THROUGH A MEDICAL SPANISH PROGRAM WITH SERVICE LEARNING AND COMMUNITY ENGAGE- MENT COMPONENTS}

Luke Hedrick, MD I Kristen Dodenhoff, BA I Priscila Arellano, MSc I Tiffany Shin, MD

Beth Israel Deaconess Medical Center/Harvard Medical School I Wake Forest School of Medicine

PURPOSE: Forty-one percent of Hispanics in the United States have limited English proficiency (LEP). Language discordance results in poor patient-clinician communication and disparities in healthcare. Medical Spanish education seeks to address health disparities by improving medical students' patient-centered communication skills in Spanish. This study assessed the effect of the preclinical portion of a longitudinal medical Spanish certificate program (MAESTRO) on student beliefs that could impact their interactions with patients with LEP.

METHODS: Nine students enrolled in the program's first cohort. The curriculum included workshops, standardized patient interactions, technology-based learning, community service, and an optional community engagement opportunity. Eight students completed the preclinical portion of MAESTRO and participated in optional community engagement. Pre- and post-surveys of these students assessed their self-reported understanding of social determinants of health (SDOH), comfort working with LEP and immigrant patients, and knowledge of community resources.

RESULTS: Before entering the program, all respondents reported comfort recognizing $\mathrm{SDOH}$ in general. However, $75 \%$ of pre-survey respondents reported comfort recognizing SDOH in their own community compared to $100 \%$ of postsurvey respondents. Fifty percent of pre-survey respondents reported feeling comfortable caring for newly arrived immigrants, compared to $87.5 \%$ of post-survey respondents. The percentage of students reporting feeling comfortable caring for LEP patients and those of varying immigration status increased by $25 \%$ (62.5\% pre, $87.5 \%$ post). Awareness of community resources increased. All respondents reported cultural considerations are important in healthcare and that the community engagement opportunity increased their cultural competency.

CONCLUSIONS: Early survey data suggests that completion of the preclinical portion of MAESTRO and the associated community engagement opportunities increased student understanding of local SDOH, comfort with LEP and immigrant patients, and knowledge of community resources. Limitations include a small sample size and limited survey anchors. Future work should refine the survey instrument and use multiple cohorts' responses.

\section{PRE-CLINICAL VACCINE MODULE TO INCREASE LEARNER PROFICIENCY WITH EVALUATING VACCINE RESEARCH AND STRENGTHEN THE CLINICIAN-PATIENT VACCINE CONVERSATION}

Michelle Demory Beckler I Amanda J. Chase

Dr. Kiran C. Patel College of Allopathic Medicine, Nova Southeastern University, Fort Lauderdale, FL

PURPOSE: Recent rises in the incidence of vaccine-preventable illnesses and public apprehension regarding vaccination is considered a consequence of an abundance of 
misinformation circulated through the media. Accordingly, it is important that future clinicians are better equipped to understand vaccine-related literature and develop skills in having difficult conversations with patients. The purpose of this module was to facilitate critical analysis of the strengths and weaknesses of vaccine-related studies, to discuss true contraindications for vaccination and to aid students in the communication of vaccine truths.

METHODS: This module incorporated different active learning approaches to maximize an understanding of primary vaccine literature as well as engage students in clinician-patient communication. Pre-reading consisted of the since retracted, though unknown at the time to students', Wakefield, A et al., manuscript that suggested the MMR vaccine is associated with autism. A pre-survey inquired about students' vaccine perceptions and practices. The module consisted of three parts in which students analyzed vaccine manuscripts, discussed myths and contraindications, and engaged in role-play. A post-survey assessed their satisfaction with the session and re-assessed their vaccine perceptions.

RESULTS: We present approaches for the incorporation of a curricular thread focused on vaccine research and communication for graduate and medical students. In addition, we present multi-cohort outcomes of student perceptions of and clinical communication about vaccination pre- and post-vaccine module delivery. Student satisfaction was high as indicated by post survey questions meant to evaluate student perceptions of engagement. Students indicated that they wish to spend more time assessing vaccine literature and engaging in role-play.

CONCLUSION: Our data suggests that both graduate and medical students benefit from approaches to address vaccination knowledge, perceptions, and communication early in health professions education. In addition, data showed evidence of vaccine hesitancy in students prior to module delivery. Approaches to augment discussion of vaccination concerns are essential to promote vaccine acceptance in future physicians.

\section{THE IMPACT OF PRIOR HEALTHCARE EMPLOY- MENT ON FIRST-YEAR MEDICAL STUDENTS' KNOWLEDGE AND ATTITUDES TOWARDS OPIOID USE DISORDER}

Lauren Culver I Tabitha Moses I Paige Baal I Tara Gloystein I Shabber Syed I Dr. Eva Waineo I Dr. Mark Greenwald

Wayne State University School of Medicine I Department of Psychiatry and Behavioral Neurosciences, Wayne State University School of Medicine
PURPOSE: Drug overdose is the leading cause of accidental death in the United States, resulting in 67,367 deaths in 2018 (CDC), nearly $70 \%$ of which involved opioids. As strategies develop to combat opioid use disorder (OUD) and overdose, research suggests continued gaps in medical education. Our goal was to study how previous paid employment in healthcare impacted knowledge and attitudes of first-year medical students towards opioid overdose.

METHODS: We invited first-year medical students at the Wayne State University School of Medicine to complete a 15-min online survey. Participation was voluntary and confidential. Information obtained included demographics, previous experience with naloxone and OUD, and Opioid Overdose Knowledge and Attitudes Scales (OOKS and OOAS, respectively). Independent $\mathrm{t}$-tests were used to compare results between groups (previous vs. no previous healthcare employment).

RESULTS: A total of 250 students (50\% female, mean age: $23.38 \pm 2.49$ years) completed the survey. Of these, 164 (65.6\%) reported previous paid experience in healthcare. No significant differences were found between students with prior healthcare employment and those without on 3 of 4 OOKS subscales: overdose risk factors $(p=0.091)$, signs of overdose $(p=0.281)$, and actions to take during overdose $(p=0.804)$. There was a significant difference in the naloxone use knowledge subscale $(\mathrm{p}=0.019)$. Overall, students with previous healthcare employment felt more competent $(\mathrm{p}=0.002)$ and reported fewer concerns $(\mathrm{p}<0.001)$ managing an opioid overdose. Interestingly, there was no significant difference between groups in readiness to intervene in an opioid overdose $(\mathrm{p}=0.057)$.

CONCLUSIONS: Students entering medical school with previous healthcare employment had more knowledge about naloxone use and better overall attitudes towards opioid overdose management. While early exposure to OUD had a positive impact, both groups lacked confidence in ability to treat opioid overdose. This study highlights a deficiency in medical education that merits further attention.

\section{ESTABLISHING A MENTORING PROGRAM FOR HEALTH SCIENCE EDUCATORS IN A NEW MEDI- CAL SCHOOL}

\section{Beatriz Tapia | Karina Madrigal | Robert Nelson}

The University of Texas Rio Grande Valley

PURPOSE: New Medical Schools need mentoring programs to enhance the personal and professional development of mentees and mentors. The need to establish a mentoring support mechanism is critical. This poster will share our progress to date. 
METHODS: A pilot mentoring program was established for junior faculty. These faculty members were paired with an associate professor or professor to serve as mentors for career development and engage in a focused scholarly project over a nine-month period. Typically, the junior faculty is within the first three years of appointment and hold the rank of assistant professor. Each mentee will have $10 \%$ protected time for this program. Our mentoring program consists of the following requirements: 1) Develop an Individual Development Plan (IDP); 2) Schedule regular meetings with Mentor; 3) Attend faculty development mentoring activities; 4) Present a draft of a scholarly project; 5) Respond to surveys and evaluations; and 6) Attend the Graduation Ceremony. The mentoring program will be evaluated by mentee performance on Individual Development Plan (IDP), mentee-mentor meeting attendance, mentee participation in mentoring activities, mentee scholarly project presentation, mentor-mentee survey feedback, and mentoring program completion.

RESULTS: We will outline the benefits, challenges, and future implications of this pilot mentoring program. Mentors foster the opportunity to excel in academic medicine in clinical, teaching, and research. Mentees will serve as junior faculty champions for future mentees participants of the mentoring program.

CONCLUSION: A mentoring program is critical in Schools of Medicine and Health Science Center. We have seen that with formal mentoring programs, junior faculty have a higher recruitment, retention rate and are committed to the mission and vision of their institution. We will take the lessons learned and address gaps in the planning and implementing this mentoring program for future cohorts.

\section{A MIXED METHODS EXPLORATION OF FACTORS THAT INFLUENCE STUDENT PARTICIPATION IN OPTIONAL FORMATIVE REVIEW QUIZZES}

\section{Jessica Morgan Jones}

\section{Georgetown University}

PURPOSE: Weekly formative Review Quizzes are an integral feature of the Georgetown University School of Medicine (GUSOM) foundational curriculum. The Quizzes offer students an opportunity to test themselves in a low-stakes setting and then discuss their answers with peers in small groups; faculty are also present to help the groups with difficult problems. The activity was designed based on the social constructivist educational framework, which predicts that discussion with peers is critical to the learning process.

METHODS: We conducted a mixed methods study in which we monitored quiz attendance over the course of the first four curricular blocks, deployed a study specific survey, and held focus groups to determine the factors that influenced quiz participation and how students perceived that the quiz contributed to learning.

RESULTS: We observed that Quiz attendance, while initially robust, dropped steadily over the course of the M1 year. Students reported that the practice questions along with faculty explanations contributed strongly to their learning, and agreed that questions should reflect the style and difficulty level of summative exams. While fewer students felt that discussion with their peers was valuable, those students who valued peer discussion were significantly more likely to attend the quiz regularly. The two things cited most often as barriers to quiz attendance where inconvenience and lack of adequate preparation. Many students reported that they saved questions and did not attempt to answer them until they had completed study of that subject.

CONCLUSION: Our results indicate that while there is ample evidence that early review and discussion with peers can contribute to learning, our learners do not always recognize the value in this practice.

\section{A WELL-DESIGNED ULTRASOUND ELECTIVE COMPLEMENTING THE UNDERGRADUATE MEDI- CAL CURRICULUM}

Kenneth Leung, M.S. I Olivia Coiado, Ph.D.

Carle Illinois College of Medicine

PURPOSE: Although most medical schools have incorporated ultrasound training into their curriculum, as there are no adopted national standards, schools have variances in the ultrasound applications covered. Offering an ultrasound elective covering any gaps identified would be beneficial.

METHODS: The Carle Illinois curriculum was thoroughly reviewed to assess for area gaps in attaining ultrasound acquisition and interpretation skills. Interpretation skills of ultrasound studies are frequently assessed in problembased learning sessions during the pre-clinical phase. Students have imaging acquisition experience on ultrasoundguided vascular access, identifying kidney pathology, and performing abdominal ultrasounds in their pre-clinical cardiology, renal, and gastrointestinal blocks. During the clinical phase, students have one week during the OB/GYN clerkship observing obstetric ultrasounds and one week on cardiology service interpreting cardiology ultrasound studies. The assessment revealed limited opportunities in attaining deeper understanding of ultrasound applications not in these specialties. A clinical elective addressing the gaps was proposed.

RESULTS: A 4-week clinical ultrasound blended-learning elective was designed. Students complete 20 different online video modules for a deeper understanding in ultrasound and 
are assessed with a quiz at the completion of each module. Students enrolled have access to a mobile ultrasound device to scan themselves; this offering significantly enriched the learning experience. Students also rotate full days with physicians who utilize ultrasound in emergency medicine, hospital medicine, critical care, and radiology, as well as with sonographers. In addition to specialties already covered in the core curriculum, these specialties identified commonly use ultrasound in their clinical practice. During COVID-19 pandemic, the in-person rotation component of the elective was suspended. Completion of this elective have shown student competency in ruling out pneumothorax, DVTs, ureteral obstructions, and retinal detachments using ultrasound. CONCLUSION: Medical schools looking to strengthen their ultrasound training should consider offering an ultrasound elective complementary to their core curriculum after a thorough assessment of content gaps.

\section{ANATOMICAL SCIENCES TEACHING ELECTIVE: A FOURTH-YEAR LONGITUDINAL ELECTIVE THAT PROVIDES TEACHING OPPORTUNITIES FOR GRADUATING MEDICAL STUDENTS}

\author{
D.J. Lowrie Jr. I Andrew Thompson | Heather Christensen | \\ Aaron Marshall I Bruce Giffin
}

\section{University of Cincinnati College of Medicine}

PURPOSE: Many medical schools are reevaluating their fourth-year curricula, including a focus on providing students meaningful preparation for residency. The ability to teach is often overlooked yet very important skill in both residency and beyond. To help prepare students to be teachers, a longitudinal fourth-year elective was developed that focuses on educating students on pedagogy and providing teaching experience in the context of the anatomical sciences. The purpose of this study is to 1) describe the elective, and 2) evaluate prior experience and motivation of students who enrolled in the course.

METHODS: The Anatomical Sciences Teaching Elective was first offered in academic year 2019-2020. At the start of the course all students $(\mathrm{N}=56)$ were asked to fill out a pre-course survey developed by the authors. The survey contained a series of questions that gauged prior teaching experience, knowledge in teaching methodology, and familiarity with education research. As a control, students enrolled in a fourth-year anatomy dissection elective were also asked to fill out the same survey.

RESULTS: Experience utilizing educational research literature was more common in students taking the dissection elective $(85 \%)$ compared to those in the teaching elective $(59 \%)$. Students enrolled in the teaching elective were, overall, less familiar and less confident with almost all teaching methods (e.g., team-based learning) compared to students enrolled in the dissection elective. Not surprisingly, $96 \%$ of students in the teaching elective felt the course would help prepare them to teach during residency compared to $82 \%$ of students in the dissection elective.

CONCLUSION: A fourth-year teaching elective was created to provide students with experience in teaching methods in preparation for residency. Survey results suggest that many students enroll in the elective because they are inexperienced in teaching and appreciate the fact that better preparation will be advantageous during residency.

\section{CONNECTING BIOCHEMISTRY KNOWLEDGE TO PATIENT CARE: A QUALITATIVE STUDY OF FOURTH-YEAR MEDICAL STUDENTS' PERCEPTIONS}

Tracy B. Fulton I Sally Collins | Marieke van der Schaaf | Bridget O’Brien

University of California, San Francisco, San Francisco CA, 94143, USA I Utrecht University, Utrecht, Netherlands

PURPOSE: Despite curriculum design intended to improve application of biomedical science knowledge to clinical practice, students struggle with knowledge transfer in clerkships. More explicit connections between biomedical knowledge gained in the classroom and workplace activities may support transfer. Our study draws on Peters' sociocultural framework of phases of transfer to explore connections students made between their biochemistry knowledge and patient care activities, their conceptions of student versus educator responsibility, and facilitators and barriers in the classroom and workplace learning environments.

METHODS: We interviewed eleven 4th-year medical students at UCSF. Participants had completed the preclerkship curriculum, core clinical clerkships, and USMLE Step 1. We took a generic qualitative approach, applying thematic analysis to identify themes related to Peters' transfer framework. RESULTS: Students reported they did not expect to use biochemistry knowledge during patient care, and rarely did so. However, all students had stories in which a connection to classroom biochemistry knowledge enhanced their learning in the workplace. Classroom factors such as emphasis on concepts relevant to clinical problems facilitated connections for some. Others noted that the minimal time for classroom biochemistry left them with a shaky knowledge base which was a barrier to making connections later. In the workplace, clerkship culture, supervisors' knowledge and attitudes regarding biochemistry, and students' perceived responsibility influenced connections. Many students described the post-core clerkship Step 1 study of biochemistry as disconnected from both their classroom and workplace learning. 
CONCLUSIONS: Students make rare connections to their biochemistry knowledge during patient care. Supervisors drive them to make these connections, though many see themselves as equally responsible. Instructional design and faculty development efforts could generate opportunities to build and strengthen these connections.

\section{STUDENT PERCEPTIONS OF A MEDICAL CURRIC- ULUM WITH FULL TEMPORAL INTEGRATION OF FOUNDATIONAL SCIENCE AND CLINICAL SKILLS TRAINING: A PHENOMENOGRAPHIC STUDY}

\section{Judith Aronson I Carol Wiggs}

\section{University of Texas Medical Branch School of Medicine}

PURPOSE: UTMB's pilot competency-based medical curriculum (SEA CHange) featured thorough integration of foundational science teaching with clinical experiences. The scarcity of literature on medical student experience of integrated curricula prompted us to study SEA CHange students' perceptions of their medical school training.

METHODS: This qualitative, phenomenographic study was conducted by a co-director of the curriculum (JA) and a $\mathrm{PhD}$ nurse educator and qualitative researcher who was not involved in SEA CHange (CW). Student input was obtained through a semi-structured group interview of the entire first cohort of eight SEA CHange students in their final semester. Transcripts were reviewed to derive units of meaning, which were then compared and grouped into categories to comprehensively describe the "outcome space" and represent critical variation in the data. The first author's "pre-understanding" (deep involvement with students and curriculum) enriched the interpretation and was balanced by the senior author's qualitative research experience and objectivity.

RESULTS: We discovered four categories of student understanding of their medical school experience. 1) The Process of Learning incorporated individual learning actions, curricular structure contributions, and interpersonal interactions (advising, mentoring, and collaboration). 2) Affective Experience included spontaneous expressions of emotions experienced, ranging from joy, humility, and confidence to struggle, challenge and frustration. 3) Learning about self and others comprised student reflections about their own growth and ability to see things from the viewpoints of patients, fellow students, or faculty. 4) Utility and value described perceptions of the usefulness of the curriculum in achieving twin goals of career development and preparation for patient care. Students reflected with fresh perspectives on the overall value of foundational science in patient care. CONCLUSIONS: The outcome space of student perceptions outlined an integrated experience of personal and professional growth in which mastery of foundational science played a supportive, but minor, role.

\section{THE TRANSITION FROM LECTURE-BASED TO STUDENT-CENTERED MEDICAL SCHOOL CUR- RICULA IN THE PRE-CLINICAL YEARS}

Joshua Burshtein I Aaron Burshtein I Dr. David Elkowitz, DO

Donald and Barbara Zucker School of Medicine at Hofstra/ Northwell

PURPOSE: Medical school core curricula have shifted emphasis from a conventional, lecture-based curriculum to a student-centered approach with the aim of applying biomedical facts to clinical scenarios and fostering problem-solving skills in the preclinical years. We describe the process of modernization of the core curriculum, from lecture-based, instructor-centered environments to student-centered, problem-solving environments.

METHODS: A retrospective review was conducted of over 40 publications, including journal articles, books, and multimedia. This literature focused on various learning methods, assessment techniques, and undergraduate medical education curricula. Our analysis details the pedagogical transition from lecture-based to student-centered learning and compares the methodologies based on their structure and application. Themes under investigation include traditional lecture-based learning and student-centered learning, including case-based learning (CBL), problem-based learning (PBL), team-based learning (TBL), and self-directed learning (SDL).

RESULTS: In 2018, AAMC found that 124 medical schools indicated a curriculum change was planned or had been implemented within the prior three years. The same analysis showed $66 \%$ increased use of case-based curricula, $38 \%$ increased use of team-based learning, and $52 \%$ increased self-directed learning. Moreover, our research has established that the shift to the student-centered pedagogies offers superior engagement with learning and application as compared to the traditional model that places emphasis on passive acquisition of material. Furthermore, our research also shows that students develop competencies such as problem-solving skills, applying biomedical science to clinical scenarios, and self-directed learning.

CONCLUSION: In the age of exponential technological advancement where facts can be acquired instantly, medical schools have transitioned from a traditional lecture-based curriculum to curricula that foster applying biomedical science and problem-solving skills through student-centered learning. Our research has shown that majority of medical 
schools have put curriculum in place in order to achieve this goal.

\section{STUDENT ENGAGEMENT IN THE CQI PROCESS: HOW USING KOTTER'S EIGHT-STEP MODEL LED TO A COURSE IMPROVEMENT}

Bailey MacInnis | Lidija Barbaric | Iuliana Balascuta | Donald Greeley MD I Kashif Ahmad MD PhD

Carle Illinois College of Medicine

PURPOSE: Medical Schools are committed to maintaining compliance with the Liaison Committee for Medical Education (LCME) accreditation standards. Creating a culture of continuous quality improvement (CQI) though evidencedbased learning is an important aspect of maintaining LCME accreditation status. While several schools have published their approaches to CQI, student involvement in the CQI process is incompletely explored. At Carle Illinois College of Medicine, an allopathic medical school that opened in 2018 and the world's first engineering-based medical school, we are committed to involving students in the CQI process. By using the Kotter's 8 step model for change management, Carle Illinois students were able to engage in the CQI process and help transform Carle Illinois's respiratory basic sciences course from the inaugural teaching of the course in 2018 to the second iteration in 2019.

METHODS: The authors describe a method of frequent, granular feedback collection rooted in Kotter's 8 step model. The authors also explain how this model can promote student involvement and buy-in in the CQI process.

RESULTS: By harnessing Kotter's 8 step method in conjunction with the student perspective, Carle Illinois's Respiratory course directors were able to quickly identify and implement necessary changes to the school's respiratory curriculum. Changes included restructuring of the course's pathology and radiology lectures to improve content retention, rearranging the to alleviate unnecessary stress, and adding various activities to ensure full coverage of necessary content.

CONCLUSIONS: Buy-in from all stakeholders, especially medical students, is an important aspect of an effective, engaging, and transformative CQI process. We believe that by expressing their voice, medical students are becoming active participants in the betterment and advancement of medical education.

CHALLENGES FACED BY THE FACULTY IN IMPLEMENTATION OF COMPETENCY BASED MEDICAL EDUCATION CURRICULUM IN INDIA

Dr Manjunatha S.N.
Mysore Medical College and Research Institute

PURPOSE: India has rolled out competency based medical education (CBME) from 2019, which meant thorough overhaul of pedagogical and assessment methods. Several new components are introduced which require focused faculty training and hand holding at times. Medical Council of India had prepared a meticulous road-map for this and dedicated faculty development programs were initiated for the smooth and effective transition into CBME. However many challenges for faced by the faculty and we wanted to study the same.

METHODS: A cross sectional survey of the faculty was done using Google forms. 189 faculty from across the country participated in the survey. The study instrument was pretested and semi structured. The instrument contained questions related to faculty training and support systems, physical and human resource infrastructure, technology support and student engagement strategies. Frequency distribution was used for presentation of data.

RESULTS: $81 \%$ of the faculty felt they faced multiple challenges in the implementation of CBME. 74\% felt the faculty strength is not sufficient to carry out all components of CBME. $63 \%$ felt lack of physical infrastructure, $59 \%$ felt inadequate faculty development programs and 53\% felt lack of support from the management. Increased small group teaching learning sessions, carrying out integrated sessions, skill training, formative assessment and documentation were considered important challenges.

CONCLUSIONS: Successful implementation of CBME in India has multiple challenges which need to be addressed at the earliest. Increasing faculty number and robust faculty development programs are required.

\section{DEVELOPMENT OF A LONGITUDINAL M4 ELEC- TIVE IN BIOLOGICAL RHYTHMS AND MEDICINE: FROM BENCH TO BEDSIDE}

Lauren A. Fowler

USC School of Medicine Greenville

PURPOSE: At USCSOMG, the curriculum seeks to integrate clinical and foundational sciences, but these opportunities are limited in the 4th year of undergraduate medical education. In 2020 a longitudinal elective was established to promote application of primary research related to biological rhythms and their influence on human health and disease. The Biological Rhythms in Medicine course was designed to allow students with clinical experience to apply foundational concepts from the biomedical sciences and research in biological rhythms to their experiences in the clinic. 
METHODS: Course content was developed to emphasize concepts related to current trends in health and disease. Concepts covered included (among others) chronopharmacology, chrononutrition and exercise, shiftwork, sleep, and the role of rhythms in chronic diseases, including obesity, diabetes, and age-related diseases. Emphasis was placed on the application of knowledge, gained through readings from current research. Active self-assessment of biological rhythms by the students was also included.

RESULTS: Sixteen 4th year medical students participated in the first year of this course. Journal article reviews, discussion boards and class discussions were used to allow students to demonstrate knowledge gained. Final presentations allowed students to integrate and personalize what they had learned and apply it to their future clinical practices. Public Outreach Briefs were developed in both written and visual formats to encourage students to explore ways to explain the role of biological rhythms in health and disease with their future patients. All students indicated that they gained new knowledge from the course, and $94 \%$ of students plan to incorporate concepts learned in their future practices.

CONCLUSIONS: The development of a longitudinal elective in Biological Rhythms in Medicine provided 4th year medical students with an opportunity to apply concepts related to rhythms and health to themselves and to issues they will face in their future clinical practice.

\section{AN EARLY INTERVENTION, CASE-BASED LEARN- ING APPROACH TO LGBTQ + SENIOR HEALTH EQUITY FOR MEDICAL STUDENTS}

\section{Arkene Levy I Samiksha Prasad I Chasity O’Malley I Daniel Griffin}

\section{Nova Southeastern University}

PURPOSE: Many LGBTQ + seniors are at risk for not receiving proper healthcare due to various factors including unsatisfactory prior experiences with untrained or insensitive healthcare providers, leading to reluctance to discuss their sexual orientations and gender identities for fear of being judged or receiving inferior care; and are at increased risk for poor mental health outcomes. This study aims to provide early intervention training to first-year medical students regarding best practices for equitable healthcare for members of the LGBTQ + community through integrative small group, case-based scenarios, reflection, and discussion.

METHODS: In March 2021, student-led small groups consisting of 7-8 first-year medical students, 1 faculty member, and 1 member of the senior LGBTQ + community will engage in discussing clinical case scenarios where LGBTQ + patients are treated either appropriately or inappropriately. Faculty will facilitate discussion using "prompt" questions as needed. The LGBTQ + community member will offer perspectives on the cases and share personal experiences related to the scenarios that will stimulate discussion. To assess the impact of the training, students will take an attitude survey, and facilitators will use a rubric tailored to intended session outcomes, including students' ability to appropriately engage in discussion, their level of awareness, and their comfort regarding providing healthcare for the population.

RESULTS: An attitude survey and a rubric will be used to analyze whether this intervention results in an improvement in the students' attitude and understanding of the importance of providing equitable healthcare to LGBTQ + patients.

CONCLUSIONS: Through this immersive discussion-based activity, first-year medical students will be given this unique opportunity to engage in conversation with senior members of the LGBTQ + community. Students will directly experience the impact of how their training involving compassionate care and mitigation of biases can make a difference in the quality of healthcare available for senior LGBTQ+ patients.

\section{CHARACTERISTICS OF EARLY LEARNERS IN MEDICAL SCHOOL}

\section{Alisa Illescas I Patrick Rendon}

University of New Mexico

PURPOSE: Resistance in learning (RIL) defines a set of attitudes or behaviors demonstrated by learners in an educational setting that result in decreased learning. Literature describes the etiology as multifactorial, including learning environment, teaching style and characteristics of the learner. We set out to describe the latter at a southwestern School of Medicine.

METHODS: This is a descriptive study of MS1s and MS2s in the class of 2023 and 2019, $N=98$. The data source included Likert scale and open response. Measures included study strategies (more effective vs. less effective), academic self-efficacy (perceived readiness for USMLE Step 1), goal orientation (performance vs. mastery), and motivation for medicine (intrinsic vs. extrinsic).

RESULTS: Student's reported a mean of 29.5 (range $=6-50$; $\mathrm{sd}=11.8$ ) hours per week studying. $56 \%$ vs. $63 \%$ of student's identified with using more effective vs. less effective study strategies. $78 \%$ of students perceived themselves to be self-efficacious in their preparation for USMLE Step 1. 95\% students reported mastery-oriented goals, and $87 \%$ reported performance-oriented goals. $94 \%$ of MS1s endorsed an intrinsic motivation for medicine while $78 \%$ of MS2s endorsed an extrinsic motivation.

CONCLUSION: Early learners are beginning to use more active forms of learning, however are still using passive 
learning techniques. They maintain a strong sense of self efficacy in academic achievement and prioritize wanting to master content, yet also want to perform well. Students are intrinsically motivated at the start of medical school, citing making an impact, serving others and curiosity as their top motivation to enter Medical School. As curriculum progresses, student have a shift toward an extrinsic motivation for learning, with USMLE Step 1 as their primary focus. Motivation to learn vs. perform could be increased by making core curriculum content more relevant to USMLE Step 1.

\section{A COURSE IN MEDICAL FACILITATION: FOSTER- ING THE PROFESSIONAL GROWTH OF HEALTH- CARE STUDENTS}

\section{Hira Qureshi I Oliver Schirokauer}

\section{Case Western Reserve School of Medicine}

PURPOSE: A major aim of healthcare education is the development of compassionate providers who are adept at communicating, responsive to the personal and societal contexts of illness, and reflective about their professional role. To meet this goal, we have designed an interprofessional course for early trainees in which students will be paired with seriously ill patients alongside whom they can experience healthcare over an extended period of time.

METHODS: Medical facilitation is a service that provides support related to communication, information processing, and decision making to patients with serious illness. Our elective is designed to give a cohort of medical, nursing, and physician assistant students the opportunity to participate in the provision of medical facilitation services. These students will accompany patients to their medical appointments, see and hear how illness impacts patients and their loved ones, and explore how to provide meaningful support. Additionally, students will attend a weekly two-hour class. Half of each meeting will be devoted to activities related to communication, contexts of illness, self-awareness, partnering, and/or professional responsibility. The other half will be dedicated to discussion of the patient cases.

RESULTS: Students will submit weekly reflections that will serve as data for a qualitative study of the course's impact on how participants approach interactions with patients and how they understand their professional role. Secondary themes of interest include interprofessional identity, contextsensitive practice, and self-care. Further feedback will be solicited from students at the completion of their respective programs in order to examine the longer-term influence of the course.

CONCLUSIONS: We believe that medical facilitation can be an effective educational tool to advance personal and professional growth. We expect that students will leave our elective with a deepened desire and improved capacity to understand what it means to be on the other side of the medical encounter.

\section{THE IMPACT OF A MODIFIED Y BLOCK TO TRAINEE EDUCATION, PRODUCTIVITY, PATIENT CARE, AND BOARD PASSING}

\section{Bhanu Chaganti}

\section{Texas Tech University Health Science Center}

PURPOSE: The $\mathrm{X}+\mathrm{Y}$ scheduling model is a novel system where the inpatient blocks $(\mathrm{X})$ are separated from the outpatient blocks (Y). This study determined the impact of an $\mathrm{X}+\mathrm{Y}$ model with a modified $\mathrm{Y}$ block $(\mathrm{X}+\mathrm{mY})$ on trainee education, wellness, board passing rates, number of scholarly activities, and unanswered clinic phone notes.

METHODS: In July 2018, the Texas Tech University Health Sciences Center El Paso Internal Medicine Residency Program transitioned from the traditional scheduling model to an $\mathrm{X}+\mathrm{mY}$ model. The modifications included the addition of wellness, research, administrative, didactic, procedural, and educational days during the ambulatory blocks. Annual administrative and research, didactic and procedural training, wellness, and protected educational time were determined before and after implementing the $\mathrm{X}+\mathrm{mY}$ model. The impact on trainee productivity and patient care were evaluated by determining the number of annual scholarly activities and the number and duration of phone notes (mean \pm SEM) before and after the implementation of the modified model. Board passing rates were reported as Percentage.

RESULTS: After the implementation of the $\mathrm{X}+\mathrm{mY}$, trainees were given access to an annual $56 \mathrm{~h}$ each of protected educational, research and administrative, didactics and procedural, and wellness times. Annual scholarly activities before and after the implementation of the $\mathrm{X}+\mathrm{mY}$ model were 35 and 45 , respectively. The baseline number and duration of unanswered phone notes were $35 \pm 6.4$ and $11.2 \pm 3.4$ (days), which improved to $23.7 \pm 2.3$ and $6.8 \pm 0.6$ (days), respectively. 2-year board passing rates were $97 \%$ and $100 \%$, respectively.

CONCLUSIONS: This novel modification provides additional protected time for trainees to utilize for their education, wellness, research, and fulfill their administrative duties, which could benefit struggling trainees. Aside from its reproducibility, this model can decrease the amount and duration of unanswered clinic phone notes when paired with an effective answering system. 
STUDENTS AND FACULTY COLLABORATE VIRTUALLY TO RESTRUCTURE MEDICAL STUDENT RESEARCH

Hannah Saven I Mariana Markell I Shirley Eisner

SUNY Downstate Health Sciences University, Brooklyn, NY 11203, USA

PURPOSE: Research experience and meaningful publication are vital to a successful residency application. The transformation of Step 1 to Pass/Fail has increased pressure on students and faculty to accelerate research activities that lead to presentation and publication. SUNY Downstate has a long history of strong student-faculty reciprocal mentoring in strengthening our curriculum. Previously, the MS4 research requirement was less effective for learning research methodology and writing. Despite the emergency need for a remote learning environment, students and faculty from the College of Medicine and Graduate School expedited a required initiative for all students to participate in research and write a hypothesis driven paper before graduation.

METHODS: Prior to the pandemic, we distributed a Qualtrics survey to evaluate research opportunities and barriers in medical school. Data analysis added support to form the student-faculty group that created an MS4 required research experience project (REP). This group met weekly to create requirements and assessments.

RESULTS: Survey responses from 203 medical students provided quantitative and qualitative data on research activities, outcomes, and barriers experienced by those who had not done research. Both MS3 and MS4 students were allowed to take the REP in 2020 due to Covid-related curricular modifications. 102 students have completed the program. $94 \%$ of students that completed feedback rated the experience favorably. By March, the REP predicts that $100 \%$ of COM 2021 students will complete the program. Prior to this, the survey indicated that 198/236 students across all classes participated in research during medical school.

CONCLUSION: Student-faculty collaboration was essential to develop the REP and to advance related curriculum initiatives which give students opportunities and skills needed to begin research by the end of their first year. A larger collaborative group was established to create a longitudinal research experience over all four years and track student research and publication data to monitor our progress.

\section{STARTING MEDICAL SCHOOL REMOTELY? EXAM PERFORMANCE \& STUDENT PERCEPTIONS}

Marieke Kruidering | Rupa L. Tuan I Barbie Klein I Chantilly Apollon I Christian Burke I Derek Harmon
Department of Cellular and Molecular Pharmacology,, University of California, San Francisco I Department of Anatomy, University of California, San Francisco I Department of Technology Enhanced Education, University of California, San Francisco

PURPOSE: To evaluate the effect of remote education on undergraduate first-year medical students' learning.

BACKGROUND: The UCSF Bridges curriculum is an integrated, organ systems-based curriculum. Pre-Covid-19, students learned via multiple modalities including: cadaveric dissection labs, lectures, small groups, online videos, interactive online modules, and case-based wrap-up sessions. Summative assessments are open-ended questions (OEQ), which require knowledge application. Anatomy assessment includes OEQ and timed cadaveric identification practical exams. Here, we (1) describe the adaption of "Ground School", the first course in Bridges, to a virtual format, (2) compare student exam performance to previous years, and (3) report on student perceptions regarding these changes. METHODS: We collected MS1 OEQ and anatomy practical scores from the 2018-2019, 2019-2020 and 2020-2021 academic years. OEQs were scored by trained faculty on a scale of 1-6 using a holistic rubric: "meets expectations" $(5,6)$, "borderline" $(3,4)$, or "does not meet expectations" $(1,2)$. Identical OEQ exams were used in 2018 and 2020. OEQ scores were averaged and analyzed using an independent samples t-test. Anatomy practical scores were analyzed in SPSS software using an independent samples t-test. Survey data are being collected and comments will be analyzed for emerging themes.

RESULTS: Mean OEQ performance was not different between cohorts (mean: 88\%; SD \pm 5 for $2018 \& 2020$ ). The 2020 MS1s virtual anatomy practical exam performance was significantly higher compared to the 2019 MS1s cadaveric exam performance $(\mathrm{p}<0.001)$.

CONCLUSIONS: Remote instruction did not decrease exam performance for the OEQ-based summative examination, while the anatomy practical exam performance increased significantly, suggesting knowledge transfer can occur via remote teaching. However, we acknowledge that the practical exam formats were not equivalent. The reported stress levels for the $2020 \mathrm{MS} 1 \mathrm{~s}$ were higher, and MS1s missed "tips to succeed" typically passed along in person via peerto-peer interactions.

PRECLINICAL MEDICAL STUDENT EXPECTATIONS OF ACTIVE PARTICIPATION IN RESEARCH

Martin Schmidt

Des Moines University 
PURPOSE: While it is universally accepted that graduating medical students need to be able to utilize research findings for lifelong learning and practice improvement, the importance of actively conducting and publishing research during undergraduate medical education is less clear. The present study sheds light on the subjective and objective importance of active participation in research though analyses of student essays and residency selection criteria.

METHODS: Subjective measures: DMU-COM OMS2 students' reflective essays on "the importance of research for medical practice" were coded for stated definitive and tentative plans to actively engage in research after graduation. Essays were submitted as a required activity for professional identity formation following attendance of the keynote address at the DMU research symposium. Objective measures: Residency placement data of DMU-COM graduates from 2008 to present $(\mathrm{N}=2175)$ were aggregated by specialty and correlated with the relative importance of involvement in research as residency applicant selection criterion (2018 NRMP Program Director Survey).

RESULTS: An analysis of student essays from the DO22 and DO23 cohort ( $\mathrm{N}$ at time of writing $=284$ ) show that the majority of students have either definitive $(31.7 \%)$ or tentative $(29.4 \%)$ plans to conduct research during their careers; $38.8 \%$ do not foresee active participation in research. An analysis of DMU-COM residency placement data show that most DMU graduates are entering residencies that place below-average value on "demonstrated involvement and interest in research" as a selection criterion for interviews (88.2\%) and applicant ranking (76.9\%).

CONCLUSIONS: Our data show that a significant number of the DMU-COM students do not foresee active participation in research, and that for the majority of students an active participation in research is not among the most important criteria for success in the residency application process. These findings are useful to inform decisions on strengthening the research curriculum and will allow to tailor educational experiences to the perceived need of stakeholders.

\section{CHALLENGES IN THE DEVELOPMENT OF A DIS- TANCE LEARNING CONTINGENCY PLAN FOR AN INTERNATIONAL MEDICAL CLERKSHIP ROTATION}

Mark Hernandez | Richard Thacker | Melinda Ledbetter | Juan Felipe Alarcon

Alabama College of Osteopathic Medicine I Universidad Central del Ecuador

PURPOSE: In 2020 due to the COVID-19 pandemic traveling abroad to complete an international medical elective clerkship rotation was suspended and a distance learning contingency plan was developed to help medical students reach their highest level of competitiveness as they prepare for residency placement and performance.

METHODS: A survey helped gauge interest in Global Health. The survey was designed for participating faculty and students to evaluate their experience with completion of an alternative elective rotation. The alternative distance learning contingency plan for elective rotation encouraged student participation by attending Zoom video conferences, with a clinical instructor practicing abroad.

RESULTS: Amid the Covid-19 global pandemic, pre-clerkship medical students continued to express interest in Global Health and International Medical Rotation electives. The planning of a distance learning contingency plan presented unique challenges such as developing an adequate model to fund the activities associated with the alternative elective rotation.

CONCLUSION: During an International Medicine Elective Clerkship Rotation, students have the unique opportunity to observe the practice of medicine in a setting that will be different, both culturally and medically, from what the student may experience during traditional medical training in the USA. In 2020, the suspension of travel abroad to participate in this clerkship elective rotation, led to the development of unique ways to support the students and collaborating faculty abroad. The development of a distance learning contingency plan has allowed an alternative pathway for students to participate in elective rotations in Global Health and International Medicine during the current pandemic and for future extenuating circumstances that would prevent in person participation.

\section{AN EXPLORATION OF THE RELATIONSHIP BETWEEN FOUR COHORTS OF MEDICAL STU- DENTS' DECISISONS ABOUT CADAVER NAM- ING AND THEIR DISSECTION-BASED ANATOMY COURSE EXPERIENCE}

Anna Sharabura I John Sherrill I Tim Atkinson I Edgar R. Meyer

University of Arkansas for Medical Sciences

PURPOSE: There are limited data demonstrating how student performance or experiences might be impacted by their cadaver-naming decisions. Nevertheless, prior work showed that students who knew personal information, such as first names, about their donors performed better in gross anatomy courses than less informed students. The purpose of this study is to explore the relationship between first-, second-, third-, and fourth-year medical students' cadavernaming decisions and their performance and experiences in a dissection-based anatomy course. 
METHODS: A survey investigating their interactions with their cadavers during their dissection and anatomy courses overall was administered to first-year medical student volunteers at the University of Arkansas for Medical Sciences (UAMS). This same survey was uploaded as a link on second-, third-, and fourth-year medical students' online learning management platforms. This study was deemed exempt by the UAMS Institutional Review Board. Participant responses will be analyzed based on students' choices to name or not name their cadaver and their agreement or disagreement with their table group in the naming process. RESULTS: ANOVAs will be used to compare students' responses and performance outcomes (e.g., exam scores, final grade) while accounting for students' reported stressors and coping mechanisms during the course. Consideration of these variables will help determine more legitimately whether cadaver interactions are correlated with students' course performance. The results will help determine the efficacy of the cadaver-naming phenomenon as a means for fostering student success in gross anatomy courses.

CONCLUSIONS: Students' habits of giving names to their cadavers have shown great potential in improving the positivity of their experiences in cadaver-based anatomy courses. However, additional analyses of results in this study will determine whether such habits have positive influences on students' course performance. Future studies will examine this cadaver-naming phenomenon and its impact among different cohorts of medical and other healthcare professional students.

\section{A COMPREHENSIVE STUDENTS-AS-TEACHERS PROGRAM: DEVELOPING AND EMPOWERING FUTURE MEDICAL EDUCATORS}

\author{
Deborah Barry I Selina Noramly I Weichao Chen I Linda \\ Waggoner-Fountain
}

University of Virginia

PURPOSE: Medical students at the University of Virginia have the opportunity to act as teaching assistants (TA) during their fourth year of study. We developed a comprehensive and robust training program to develop medical students as educators in preparation for role as a TA.

METHODS: This program was developed utilizing a backwards course design, first piloted in 2017. Since then, three formats of the course have been offered, a two-week intensive in-person course meeting for 50 contact hours, a workshop series meeting for 10 contact hours, and an online intensive delivered during the COVID-19 pandemic. Each course format includes the opportunity for students to develop their teaching philosophy, practice skills-based teaching, microteaching, write and review multiple choice questions, and the complete a capstone project. Students within the intensive format courses develop their communication skills, review pedagogy related to medical education, gain additional experience microteaching, and receive additional guidance to complete their capstone project.

RESULTS: To date, 138 students have completed the training program, while 116 students have subsequently completed a TA. Students have developed high quality pre-recorded lectures, study guides, and assessment questions that have been implemented across the curricula for first- and secondyear students. Several of these projects have been created to supplement third- and fourth-year clerkships and electives as well as out-of-classroom experiences, such as diversity, equity, inclusion, wellness and stress- management. Faculty have reported on the high-quality of student created materials, as well as increased involvement of teaching assistants after their participation in the training program.

CONCLUSION: This comprehensive program designed to assist medical students gain competency in various aspects of medical education has resulted in a wide variety of student created materials that have been implemented in our curricula. Both students and faculty report an increase in teaching assistant skills as a result of participation.

\section{INTERNALIZATION OF THE PROFESSIONAL VALUES}

\section{Shima Tabatabai}

Shahid Beheshti University of Medical Sciences, Tehran, Iran

PURPOSE: Internalization of the professional values related as the main requirement for medical practice is very important just like the way these values are taught and learned. This study's aim is to investigate the medical education experts' viewpoint about professional values as a step towards integrating professional values in medical education.

METHODS: A qualitative approach was adopted for this study. The data were obtained from the panel discussions with medical education experts with a variety of experiences in Iran. All sessions were audio-recorded, transcribed, and analyzed using thematic analysis.

RESULTS: The participants expressed their views on the ways professional values should present in medical education. The data analysis revealed 4 main categories: 1) Employing effective faculty development methods, 2) Employing Educational strategies including teaching, learning and assessment methods of professional values by qualified faculty and clinical mentors, 3) Role of context and regulations to present professional values, 4) strengthening the hidden curriculum. Experts recommended that 
value-based medicine should be integrated into the general medical curriculum as a longitudinal theme. Specifically, the integration of professional values and its assessment in the clinical phase is recommended.

CONCLUSION: To address the Internalization of the professional values in medical students, the main factors, i.e., value-based Educational strategies, contextual values, and executive resources for strengthening the hidden curriculum should be considered. Developing a formal value-centered medical educations system would be necessary and integrating the professional values with all its phases recommended. Employing effective teaching and assessment methods by means of qualified faculty/clinical mentors with valuable experiences is necessary for the internalization of the professional value in medical students.

\section{AN INTEGRATED, LONGITUDINAL HEALTH EQUITY CURRICULUM TO PREPARE SOCIALLY RESPONSIBLE HEALTHCARE PRACTITIONERS}

\author{
Rupa Lalchandani Tuan I Stephanie Hsia
}

University of California, San Francisco, School of Medicine

PURPOSE: The racial injustices amplified by the COVID19 pandemic have stressed the importance of educating healthcare professionals to be advocates for social justice. We designed and implemented a ten-week Health Equity curriculum to promote structural competency and reduce implicit bias in healthcare providers. The purpose was to teach students to identify and recognize structural causes of health disparities, design interventions, and engage in equitable, civil, and compassionate discussions. Here we report on student performance and attitudes regarding this curriculum.

METHODS: The curriculum was a mandatory component of the Neuropsychiatric Theme for second year Pharmacy students. Students were assigned to subgroups of 5-6 and distributed across self-identified gender and ethnicity. The curriculum was conducted remotely and consisted of didactic material, asynchronous online discussions, and synchronous Zoom discussions. Didactic material included videos, podcasts, journal articles, and faculty-created lectures. Asynchronous discussions were structured around the didactic content and open-ended prompts were provided to stimulate conversation. Three live discussions provided students with space to discuss the topics in real time. Four main topics were covered: (1) cultural and structural influences on mental health; (2) mental health and LGBTQ populations; (3) homelessness, deinstitutionalization and the mental health system; and (4) a student-identified topic. A survey was administered before and after the curriculum, and topics were assessed in an OSCE.
RESULTS: Student responses from pre- and post-surveys will be compared and quantitatively analyzed. A thematic qualitative analysis of student responses to open-ended questions will be conducted and compared. OSCE performance will be assessed for application of what was learned.

CONCLUSION: Given the challenges of integrating health disparities education into already impacted curricula, we believe our curriculum presents a rich learning experience with minimal in-class time and has potential for wide dissemination across health professional schools. From this study, we will gain insight into student engagement and performance in an online Health Equity curriculum.

\section{EVALUATING EDUCATIONAL OUTCOMES ACROSS PRECLINICAL AND CLERKSHIP YEARS OF MEDICAL SCHOOL ANALGESIC AND OPIOID EDUCATIONAL ACTIVITIES}

\author{
Jake Bentley I Laurel Gorman
}

\section{UCF College of Medicine}

PURPOSE: Institutions have called on medical schools to improve education in pain, analgesics, and opioids. Our institution integrates pain education throughout the curriculum with pharmacology mostly in the second year (M2). Our purpose was to evaluate outcomes of our educational processes by examining initial increases in analgesic knowledge, retention in third (M3) and fourth (M4) year students, and learner's perceptions of educational effectiveness.

METHODS: We retrospectively examined performance on multiple choice questions (MCQ) weeks after activities in M2 students. We looked at retention of analgesic concepts by quizzing $\mathrm{M} 3(\mathrm{~N}=30)$ and $\mathrm{M} 4(\mathrm{~N}=36)$ volunteers months to years later. We compared M3 \& M4 results with a cohort $(\mathrm{N}=50)$ who hadn't yet experienced the opioid curriculum as the baseline. All cohorts answered survey questions on the curriculum.

RESULTS: Summative MCQ data showed that M2's initially mastered opioid and pain concepts (Mean 86\%) and retained knowledge in M3 \& M4, scoring significantly higher on concepts taught 6 months to a year previously then the baseline group (p80\% M3 \& M4), confidence (15\% baseline cohort, $67 \% \mathrm{M} 3 ; 80 \% \mathrm{M} 4)$, and awareness (12\% Baseline cohort, $60 \%$ in M3 \& M4) increased in M3/M4 cohorts in comparison with the baseline cohort. Lower scoring items included integration of basic science in clerkship and diversity.

CONCLUSIONS: Data suggested that initial analgesic knowledge increased and learners retained many concepts years later, supporting the effectiveness of analgesic educational processes. Learners' opinions were mostly positive, perceiving that the educational processes increased comprehension, confidence in treating pain, and awareness of 
tools, but many felt that educational activities were insufficiently diverse and the integration of basic sciences could be improved in clerkship opioid and pain education.

\section{FOSTERING THE DEVELOPMENT OF HEALTH PROFESSIONS EDUCATION SCHOLARS THROUGH COLLABORATIVE LEARNING \& PRACTICE}

\author{
Shari Whicker, EdD I Sarah Parker, PhD \\ Carilion Clinic/Virginia Tech Carilion School of Medicine
}

BACKGROUND: Advancement of high-quality health professions education research is dependent upon the development of educators who design research with the same rigor demanded in basic science or clinical research. To support this need, one teaching academy used a social constructivist approach to develop an interdisciplinary program comprised of a health professions education research curriculum paired with an internal resource supported and mentor-guided education research project.

METHODS: Authors developed the Health professions Education Scholars (HERS) program, designed to facilitate participants through individual health professions education research project. A competitive selection process was established to identify participants. The program curriculum included the AAMC Medical Education Research Certification (MERC) program, internally led instruction, hands-on training, and mentorship through the design and execution of a health professions education research project.

RESULTS: Eight clinicians were selected to participate in the first year of the program. Participants represented a diverse group of educators from across the health system. Participants were matched with a mentor and required to protect one designated day per month to devote to participation in the program. At the end of the first cycle, all participants received MERC certification and disseminated/ presented their projects at the regional, national, and/or international level. The second cycle of the program began with 8 participants in Fall 2019. The program was revised to an 18-month curriculum after feedback from our first cycle. DISCUSSION: The HERS program was designed to provide instruction in health professions education research principles and practical skills to do so effectively, foster collaboration within health professions education research, and develop leaders in academic medicine. This intensive, mentorship-guided program has and will continue to improve the execution of health professions education at their institution, build a coalition of skilled medical education researchers, and ultimately contribute to national and international academic research on health professions education.

\section{A TEAMWORK APPROACH TO COMPILING A REVIEW PAPER ON COVID-19 EXPERIMENTAL TECHNOLOGIES}

\author{
Md Shahnoor Amin I Olivia Coiado
}

Carle Illinois College of Medicine, University of Illinois Urbana Champaign I Department of Biomedical and Translational Sciences, Carle Illinois College of Medicine, University of Illinois at Urbana-Champaign

PURPOSE: The Carle Illinois College of Medicine is the first college in the nation designed at the intersection of engineering and medicine. Our goal is to develop a new generation of physician-innovators that can apply quantitative skills and collaborate with different teams to devise health care solutions. The goal of this study is to describe the design of a Literature Review Elective about COVID-19/ SARS-CoV-2.

METHODS: The elective was a 4-week course designed by a biomedical engineering faculty. A multidisciplinary group of faculty, post-doctorate, and medical students collaborated on this comprehensive literature review paper on experimental technologies utilized in the diagnosis and treatment of COVID-19. The objectives of the elective were: think critically about important issues related with COVID-19; critique possible therapies in the context of the current COVID-19 outbreak, enhance communication skills, and foster teamwork.

RESULTS: The initial 2 weeks were spent on literature review to understand the pathogenesis of COVID-19 and how it compares to prior epidemics and pandemics. Over the next 2 weeks, the research team conducted a thorough investigation of the literature related to diagnostic and therapeutic technologies used to manage COVID-19. Finally, the team completed the review paper, made grammatical corrections, corrected the extensive list of references, and determined authorship. The paper was subsequently submitted to a relevant journal for review in June 2020.

CONCLUSION: Students learned how to search scientific papers on online libraries and websites, and how to organize, analyze, and criticize the literature review. This elective has demonstrated the importance of continuing research collaborations across multiple departments in the midst of a pandemic, fostering a sense of comradery in an uncertain time, and compelling students to learn about a novel disease that was not in medical school textbooks.

\section{A STEP TOWARDS CHANGE: INTEGRATING HEALTH SYSTEMS SCIENCE INTO FOURTH YEAR OF MEDICAL SCHOOL}


Sarah Siddiqui I Premal Patel I Hani Serag I Samee Hameed I N. Miles Farr

University of Texas Medical Branch

PURPOSE: The COVID pandemic brings to the forefront several gaps in United States' healthcare and public health systems along with health inequities among vulnerable populations. It has never been more evident and critical for us to recognize, develop, and implement health systems science (HSS) curriculum into medical education. Whereas the traditional model has been to focus on basic and clinical sciences, the recent movement calls for integrating HSS as the third pillar. Improving the understanding of HSS among medical students will enable them to become more well-rounded physicians who can apply their foundational knowledge and improve the health of their patients and community. We describe the curriculum for a new course called "Health Systems Science \& Community Service Course (HSS Course)" at the University of Texas Medical Branch. METHODS: The HSS course is a required, longitudinal course for fourth year medical students. It consists of two key components: 1) online, self-directed learning focusing on HSS domains including, but not limited to, health system improvement, social determinants of health, and systems thinking, 2) participating in community service. The overall goal of this course is for students to increase their knowledge and understanding of the various HSS domains. Assignments include online modules, community service, reflective assignments, and tests.

RESULTS: We are currently in our first year of implementation of the course. As this is a self-directed course with mostly virtual experience, it makes it more compatible and flexible during the COVID pandemic.

CONCLUSION: While implementing this course has laid a foundation for the HSS curriculum, we recognize the importance of evaluation especially as this course is in its infancy. We plan to review course evaluations by students. Some areas of improvement we are considering for next academic year include creating virtual interactive small group activities and moving towards community service learning experiences.

\section{A SUMMER RESEARCH CURRICULUM TO CULTI- VATE MEDICAL STUDENT RESEARCH}

Jonathan Murrow, MD MSc I Amy Medlock, PhD

\section{AU/UGA Medical Partnership}

PURPOSE: Evidence-based medicine serves as the bedrock of high quality, high value health care delivery. Medical student research experience holds enduring value among educators and students alike. Despite this emphasis, research experience during undergraduate medical education varies from self-directed exposure to formal year-long curricula. METHODS: In this context, we evaluated the hypothesis that developing a formal research curriculum would improve knowledge, attitudes, and outputs among medical students. We developed this curriculum by performing a needs assessment, defining goals and objectives, determining the optimal educational strategy, and implementing the curriculum through six weekly instructional sessions delivered over a 10 week summer research elective. Pre- and post-surveys were performed to assess knowledge and attitudes that students held about research.

RESULTS: Goals and objectives of a medical student research curriculum included the need to define a research question, to develop a protocol, to analyze results, to synthesize findings, and to communicate new knowledge to a broad audience. In our cohort of students, twenty-one completed the course. The majority had no prior research experience, and many identified successful residency matching as a reason for pursuing research. Students increased confidence as a cohort in addressing research setbacks and analyzing data after completing the course. Students had high confidence in performing literature reviews and writing proposals before and after the curriculum, while endorsing low confidence in preparing grant proposals.

CONCLUSION: This novel curriculum identifies key motives that students identify in pursuing research experiences while in medical school. Formal curricular elements offer the opportunity to increase confidence in engaging research setbacks and in analyzing data. Further work should help to refine areas where students are most likely to benefit from research instruction.

\section{INTERDISCIPLINARY CHALLENGE BASED LEARNING FOR HEALTHCARE PROGRAMS}

\section{Silvia Olivares I Jorge Valdez-Garcia}

\section{Tecnologico de Monterrey}

PURPOSE: Longevity, patient-centeredness, healthy lifestyle living, and disease prevention are the most critical trends for medical education, and also the least included in medical degree programs. These curriculums are highly focused on the diagnosis and treatment of common pathologies with limited training for nutrition, mental health, and other wellness topics, which are now being exponentially overtaken by non-scientific professions and publications. The purpose was to completely reconceptualize the health care programs at Tecnologico de Monterrey to address these concerns. 
METHODS: More than 100 faculty from health sciences collaborated for two years to consense competences and challenges for patient centered learning, where no single profession had a leading role. Patient centered care, Biomedical research, Professionalism, and Resource Management were stated as the four main competences to be acquired for the students. In August 2019, the renovated programs were implemented for 546 students. The curriculum has three phases: Exploration (1.5 years), Focus (1.5-2.5 years), and Concentration (1-2 years). The pedagogical approach is Challenge Based Learning, which is intended for students to solve real healthcare challenges applying collaborative action learning methods (Marquardtand Waddil, 2004).

RESULTS: The Exploration phase includes eight common challenges for healthcare programs. The students should develop competences in prevention, potential health risks detection, and patient healthy lifestyle education; considering medical, nutritional, mental, and scientific perspectives. Examples of the challenges are self-healthy lifestyle practices, effects of pollution on molecules and cells, health risks on migration journeys, and teen pregnancy prevention, among others.

CONCLUSIONS: This learning experience closes the bridge between theory and practice on emerging critical healthcare problems at the self, patient, and society levels. Students proved that they are capable of proposing innovative solutions, even for their own health. However, they battle with time management, teamwork relations, and content detail or argumentation.

\section{ARE YOU READY?: ASSESSING EDUCATIONAL NEEDS FOR THE GENOMIC ERA OF MEDICINE}

Matthew J. Ryan I Karla Lindquist I Anica M. Wandler I Cynthia Morgan I Sawona Biswas I Elena Flowers I W. Patrick Devine I Teresa N. Sparks I Maren T. Scheuner I Bani Tamraz I Thomas Hoffmann I Joseph Sheih I Neil Risch I Katherine Hyland

University of California, San Francisco, Institute of Human Genetics, San Francisco, CA

PURPOSE: Clinical genomics is transforming the diagnosis, treatment, and prevention of disease. However, there are not enough clinical geneticists and genetic counselors to meet the increasing demand, and non-geneticist clinicians feel under-prepared to incorporate genetics/genomics into practice. We hypothesized that non-geneticist clinicians want training in order to practice and teach genomic medicine confidently, and sought to identify specific educational needs of clinicians/trainees at University of California, San Francisco (UCSF).
METHODS: In a mixed methods study, key informant interviews with 13 Department Chairs from UCSF School of Medicine using an 8-question interview guide were coded using thematic qualitative analysis. These interviews informed development of a 28 -item survey widely distributed to clinical departments.

RESULTS: Interview responses revealed that genetics/ genomics are underutilized in clinical practice, more education is needed for trainees and clinicians, and having clinicians with genetics expertise within departments is crucial to improving the utilization of genetics/genomics. Among non-geneticist physicians $(n=144)$ surveyed, $82 \%$ reported they would be more comfortable using genetics/genomics in clinical practice with additional training. Lack of knowledge was cited as the primary reason for not ordering genetic tests $(51 \%)$. Only $34 \%$ reported participating in genetics continuing medical education (CME); among those who had not participated, $65 \%$ cited lack of awareness of CME courses while $15 \%$ cited lack of need. Importantly, the vast majority $(90 \%)$ of non-geneticist physicians indicated they would benefit from additional training. The leading topics of interest included information about genetic testing options and communication of results, human genetics fundamentals, and ethical issues.

CONCLUSIONS: Lack of knowledge impedes clinicians from incorporating genetics/genomics into practice and teaching, and the vast majority of clinicians desire more training. Additional training is essential to increase the number of genomically literate clinicians and educators. Next steps include working with clinical departments to develop general and specialty-specific training materials for clinicians and trainees.

\section{REIMAGINING THE USE OF HUMAN PATIENT SIMULATOR: A BRIDGING TOOL BETWEEN SCI- ENCE LEARNING AND ITS APPLICATIONS TO THE CLINICAL ENVIRONMENT IN MEDICAL EDUCATION}

\section{Elizabeth Prabhakar}

Brunel Medical University, Brunel University, Uxbridge, United Kingdom

PURPOSE: To enable early years medical students to understand the application of foundational science to clinical practice, using a Human Patient Simulator. This will provide the learning continuum for medical students transitioning from learners to practitioners.

METHODS: Students investigated the effects of haemorrhage on a simulated male patient, $90 \mathrm{~kg}$, involved in an automobile accident. Normal blood volume $70 \mathrm{ml} / \mathrm{kg}$. The 
"patient" was bled (ml): 0, 600, 1200, 2100 and 3000. Heart rate, systolic BP, pulse pressure, central venous pressure, total peripheral resistance, cardiac output and respiratory rate were recorded on a structured worksheet. Fluid loss was calculated as a percentage of total blood volume and classified into stages I-IV of haemorrhagic shock. Shock index was also calculated.

RESULTS: Data obtained from the simulator was displayed graphically demonstrating the relationship between circulating blood volume as a function of systolic BP or heart rate; central venous pressure vs stroke volume. Cardiac output and total peripheral resistance were also analysed in different stages of shock. Students benefitted from observing the clinical features of shock like weak pulse, tachycardia, hypotension.

CONCLUSIONS: Students were able to explain their observations using their physiology knowledge and give reasonable suggestions for restoring haemodynamic stability. In later clinical years, students would be expected to engage in more complex discussions regarding management with intravenous fluid therapy and/or blood products. The graphs compelled students to think critically and make connections between foundational theory and clinical practice. The lessons learned in this report were that Flexner's $2+3$ medical model of teaching had to be disrupted to integrate the basic science and clinical science. This is a work in progress and will continue into the future, using different clinical scenarios to help learners transition to skilled practitioners. This work was carried out at the presenting author's previous institution.

\section{ASSESSMENT OF CLINICIAN AND STUDENT PER- CEPTIONS OF THE IMPORTANCE OF EMBRYOL- OGY IN THE MEDICAL CURRICULUM}

\section{Zachary Bennett I Anna Edmondson}

Medical College of Georgia at Augusta University, Augusta, GA, 30912, USA

PURPOSE: With changes to medical curricula, it is important to determine the relevance and clinical applicability of content taught. With the prevalence of congenital anomalies, understanding embryology is imperative for clinical practice. The purpose of this study is to assess clinician and student perceptions of the relevance of embryology in the medical curriculum.

METHODS: To assess perceptions of the importance of embryology in the medical curriculum, clinical faculty $(n=3)$ and 4 th year medical students $(n=24$ out of 190) completed a survey consisting of questions focused on assessing what aspects of embryology are perceived to be important for medical students to learn for clinical practice.
Numeric data were quantified, and narrative qualitative data were coded and themed using a constant comparative method.

RESULTS: 75\% of student respondents felt their knowledge of embryology was "fair" or better prior to starting their clerkships (avg. score $3.0 \pm 1.2 ; 1=$ very poor to $6=$ excellent), and $58 \%$ believed that having knowledge of embryology was important for success during clerkships (avg. $2.7 \pm 1.3$ out of 5). Among the organ systems, students believed Gastrointestinal (avg. score $3.47 \pm 0.32$ ) and Genitourinary $(3.28 \pm 0.37)$ systems embryology were the most important, while Musculoskeletal embryology was least important $(1.99 \pm 0.34$ out of 5$)$. By clerkship, $92 \%$ of students claimed they came across embryology in OBGYN and Pediatrics compared to $4 \%$ in Psychiatry. Of clerkship directors surveyed, $100 \%$ believed that knowledge of embryology was "very important" for success on clinical rotations and believe students have a good knowledge base of the clinically relevant aspects of embryology when beginning rotations.

CONCLUSION: The preliminary study shows that embryology is prevalent throughout clinical rotations, but the relative importance of different topics varies. The results of this study may be useful in developing a curriculum that focuses on the embryologic topics most frequently seen in clinical rotations.

\section{MAPPING OF THE MEDICAL UNDERGRADU- ATE CURRICULUM AT THE UNIVERSITY OF ZIMBABWE}

Shalote Chipamaunga

University of Zimbabwe

PURPOSE: There is abundant literature on the increase in numbers of medical errors and there are reports that medical education is in some instances fragmented, outdated with static curricula producing ill-equipped graduates. The University of Zimbabwe embarked on efforts to transform its under-graduate medical curriculum to meet the needs of the nation more appropriately while matching international benchmarks. This abstract describes the process taken to map the curriculum.

METHODS: Following the curriculum mapping framework, the exercise took place over three years (2018-2020). As far as possible, we explored the curriculum through these windows: learning outcomes; curriculum content; student assessment; learning opportunities; learning location; learning resources; timetable; staff; curriculum management; and students. We managed to get teachers to exchange information about what is being taught to enable us to determine the different components- 'declared', 'taught' and 'learned' 
curriculum. With a revived and reconfigured curriculum review committee, we conducted over 20 departmental meetings and workshops to enable wider collaboration with all disciplines in the Faculty. The committee convened regular, sometimes weekly meetings which ran for an average of $2 \mathrm{~h}$ each.

RESULTS: Over 120 faculty were involved in curriculum mapping. Initially there was some resistance and little appreciation for the mapping with expressions of "why this process?", "if it's not broken why fix it?", "this curriculum produces reputable and well sought after graduates so why interrogate it?", "we are products of this curriculum and we function well, don't we?". There was evidence of content overload; lack of horizontal and vertical integration; timetable variances; and missed opportunities for learning.

CONCLUSION: Curriculum mapping, first step in curriculum review, increases faculty knowledge of the program and presents gaps or opportunities for improvement.

\section{OPPORTUNITY FOR STUDENTS TO IMPROVE MEDICAL KNOWLEDGE AND RECEIVE FAC- ULTY FEEDBACK IN AN EMERGENCY REMOTE SETTING}

\section{Nina Kumar I Esther Yoo I Shirley Eisner}

SUNY Downstate Health Sciences University, Brooklyn, NY, 11203, USA

PURPOSE: In response to the COVID-19 pandemic, firstyear medical students (MS1s) worldwide attended gross anatomy (GA) lab remotely, leaving fewer opportunities to practice anatomic terminology, collaborate, and receive faculty feedback. To address these concerns, we implemented the Recorded Dyad Presentation (RDP). This allowed MS1s to enhance their communication and anatomy skills safely and to prepare for peer-teaching during in-person progressive dissection.

METHODS: The RDP was assigned to $210 \mathrm{MS} 1 \mathrm{~s}$ during a 5-week remote GA curriculum on the musculoskeletal system. Each 3-h Zoom session (34-37 MS1s) was led by one GA professor and held 1-3 times per week. To implement the RDP: (1) advise students to choose a partner and sign up to present; (2) provide a manual with goals, requirements, expectations, and guide to record on Zoom; (3) set clear due dates; (4) advise students to record and submit presentations; and (5) provide standardized feedback.

RESULTS: The student survey, sent to $210 \mathrm{MS} 1 \mathrm{~s}$, received 88 responses. Thirty-three of these students received faculty feedback (Feedback) and 55 did not (Control). The following percentages reflect those who "strongly agree" or "agree". In the Feedback group, $60.6 \%$ reported improved anatomy understanding, ability to use correct anatomic terminology aloud, and oral presentation and communication skills, and $84.8 \%$ learned from faculty feedback. In the Control group, only $43.6 \%$ reported improved anatomy understanding, $45.5 \%$ ability to use anatomic terminology, and $38.2 \%$ oral presentation and communication skills. The Feedback (72.7\%) and Control (61.8\%) groups both enjoyed working with a partner. Data analysis will be completed for final presentation.

CONCLUSIONS: The RDP promotes peer-teaching, collaboration, and professional development in a remote environment. Providing students with feedback is imperative to successfully implement the RDP and to derive the pedagogic benefits.

\section{COMPARING EDUCATIONAL EQUIVALENCY OF INTERNATIONAL CLINICAL ROTATIONS}

\author{
Nicole Dettmann, DSc, MPH, PA-C
}

\section{MCPHS University}

PURPOSE: In recent years, there's been rapid growth of international medical rotations. A survey conducted by the Physician Assistant Education Association in 2018 reported over 159 Physician Assistant students from 99 institutions participated in international rotations. There is little analysis of the quality of these experiences. One question asked is can "core rotations" be completed internationally? This research will compare the educational equivalency of international clinical rotations and clinical rotations in the United States.

METHODS: From a 2018 class of 125 PA students, 10 students $(n=10)$ or $8.65 \%$ participated in international Women's Health (WH) rotations in Bolivia. Data from rotations was used to compare these students with students who completed WH in the US. Scores for the WH end-of-rotation exams (EORE), preceptor, and student evaluations were collected for data analysis. Descriptive statistics were calculated for both domestic and international groups. Mean scores were compared using t-tests for interval data and Mann Whitney $U$ tests for ordinal data.

RESULTS: No statistical significance $(\mathrm{p}<0.05)$ observed between the two groups for the mean scores in these three categories. The significance between groups for the EORE mean scores was 0.162 . The significance between groups for the 18 sub-topic scores of the preceptor and student evaluations ranged from 0.184 to 0.716 .

CONCLUSION: Results from this cohort show no significant statistical difference between the groups when comparing the data of EOREs, and preceptor and student evaluations. This demonstrates no statistical difference in the educational quality of the rotations between the WH students in Bolivia and the US. When properly designed and 
implemented, these findings support participation in international rotations, which can provide strong educational value and should be considered for use of core rotations.

\section{MEDICAL STUDENTS' PERCEPTIONS REGARD- ING USMLE STEP 1 STUDY RESOURCES}

\author{
Emily Hansinger | Cecillia Lee I Nagaswami S Vasan | \\ Cheryl A. Melovitz-Vasan
}

Cooper Medical School of Rowan University I Department of Biomedical Sciences, Cooper Medical School of Rowan University

PURPOSE: Achievement in the USMLE Step 1 examination is a milestone for medical students. Examination scores are routinely used to screen for residency interviews, thus creating increased stress on students to perform well. This has resulted in increased use of commercial resources to prepare for the Step 1 examination. The purpose of this study was to identify factors that influence medical students' decisions to use commercially available Step 1 resources.

METHODS: An anonymous survey was sent to $227 \mathrm{~s}$ and third year medical students at Cooper Medical School of Rowan University during the fall of 2020. The survey contained closed-ended questions regarding commercial resources used for Step 1 preparation, money willing to spend on resources, motivation for using resources and the school's role in preparing students for Step 1.

RESULTS: Sixty-three students $(27.8 \%)$ responded to the survey. Fifty-one percent of students use 3-4 commercial resources. Sixty-eight percent did not set a total dollar limit on what they would spend on resources, with $43 \%$ spending between $\$ 300.00-\$ 600.00$ dollars. First Aid (95.2\%), UWorld (85.7\%), Pathoma videos (85.7\%), and Anki flashcards $(85.7 \%)$ were the most frequently used resources. Price (50.8\%), and peers (36.5\%) influenced students' decisions to purchase commercial resources. In addition, students indicated they used commercially available resources to: manage study time (53.9\% strongly agree), alleviate anxiety (36.5\% strongly agree), and supplement the school's curriculum ( $57.1 \%$ strongly agree).

CONCLUSIONS: To the best of the authors' knowledge, this study is the first to identify factors that influence medical students' choices regrading the use of commercially available Step 1 resources. Among the choices, price, peers, management of study time and anxiety, as well as curriculum supplementation were all found to be influential factors in the decision to purchase and use commercially available Step 1 resources.

\section{PERCEPTIONS OF LEADERSHIP AND PROFES- SIONALISM VALUES AMONG MEDICAL STU- DENTS IN GROSS ANATOMY DISSECTION}

Tanner Stumpe I Samantha Benevides I Anna Edmondson, $\mathrm{PhD}$

Medical College of Georgia at Augusta University, Augusta, GA 30912, USA

PURPOSE: Patient outcomes are highly impacted by the extent of collaboration between the healthcare team. Along with anatomical expertise, anatomy dissection provides opportunities for students to gain insight on personal leadership style, attain robust interpersonal skills, and develop clinical competencies. This project's aim was to determine the medical student perceptions and attitudes concerning gross anatomy dissection in order to assess evolution of core values, attainment of professional skills, and collaborative capabilities that are critical for success as a medical provider.

METHODS: First-year medical students $(n=106$ out of 190) completed a survey assessing perceptions and attitudes of gross anatomy dissection upon completion of the firstyear curriculum. The survey consisted of Likert, narrative response, and multiple-choice questions. Students $(n=14)$ also participated in semi-structured focus groups. Content analysis for the focus group data was performed using the constant comparative method for theming and interpretation. RESULTS: Of students surveyed, 63\% agreed or strongly agreed that dissecting with other students helped them develop leadership skills (avg. $4.29 \pm 0.46$ ), and $73.5 \%$ of students agreed or strongly agreed that their fellow dissection group members possessed good leadership skills (avg. $4.37 \pm 0.49$ ). $73 \%$ of students found that team dissection helped facilitate learning how to interact professionally (avg. $4.27 \pm 0.45$ ). $81 \%$ of students that agreed or strongly agreed that their group members possessed good leadership skills also reported that dissection made them think more critically about location and function of anatomical structures. In focus group sessions, $100 \%$ of participants perceived dissection had a positive impact on enhancement of leadership and/or professionalism skills.

CONCLUSION: The data show that cadaveric dissection serves a unique role in enhancing student interaction and promotes a space for leadership and professional skill development. Further development of this hidden curriculum involving cadaveric dissection may be helpful to ensure collaboration and professional interaction. 
ASSESSMENT OF FIRST YEAR MEDICAL STUDENTS' COGNITIVE LEARNING STRATEGIES OF THE CRANIAL NERVES

Shahar Laks I Anna Edmondson, PhD

Medical College of Georgia at Augusta University, Augusta GA 30912, USA

PURPOSE: Medical students often have a difficult time understanding the cranial nerves and transferring that knowledge to clinical practice. Students often resort to rote memorization instead of cognitive learning strategies, or may avoid focusing on the content altogether. This study's aim was to assess medical student cognitive learning strategies in regards to cranial nerve anatomy and its related clinical applications.

METHODS: First year medical students $(n=168$ out of 192) were asked to complete a survey at the end of the curriculum to assess their knowledge, clinical application, and approach to learning cranial nerves. Students $(n=12)$ were subsequently recruited to participate in virtual semi-structured focus groups to further assess their cognitive learning strategies. Comments from surveys and focus groups were themed, quantified, and analyzed.

RESULTS: $52 \%$ of students found learning the cranial nerves difficult or very difficult, compared to $41 \%$ neutral and $7 \%$ easy or very easy. The most common explanations for student perceived difficulty levels included the volume of material and amount of small details for the very difficult/ difficult group (11\% and 19\% of respondents respectively), improvement with time and spaced repetition for the neutral group (11\% and $8 \%$ of respondents respectively), and previous background knowledge for the easy/very easy group (45\% of respondents). When approaching information for the first time, students focused on creating associations (21\%), compiling all the information into summary tables (23\%), and focused on class lecture material (47\%). 59\% of students who participated in focus groups stated that the biggest obstacle to learning was transitioning to online learning due to COVID-19.

CONCLUSION: Medical students struggle with the large quantity of material, though they note improvement with spaced exposure. Medical curricula should focus on incorporating metacognition and active learning strategies to promote improved learning techniques.

\section{THE PROCESS OF CREATING NEW CURRICULAR CONTENT THROUGH FACULTY-STUDENT FEED- BACK AND NEGOTIATIONS}

Linda Chang I Radhika Sreedhar I Paul Chastain II | Jeffrey J.H. Cheung
University of Illinois College of Medicine at Rockford

PURPOSE: The ever-evolving nature of medical practice means that teaching faculty will inevitably need to adapt curricular content to reflect these changes. Faculty then must create content that reflects knowledge and skills that future clinicians will need to know, but also work with students to refine and optimize these new educational experiences. This process of iterative refinement is underscored by the potential for misalignment between what faculty and student deem valuable or preferable. We describe how faculty used student feedback during a 3-year process of creating, introducing, evaluating, and adapting an Evidence-Based Medicine (EBM) course in our new curriculum.

RESULTS: As we were assessing students feedback for EBM from the first year of our new curriculum (2018), we noticed that while students were successful in answering EBM questions $91 \%$ of the time and that faculty reached goals and learning objectives for their sessions, student comments suggested that they were unsatisfied with the class as a whole (general comments were why are the sessions mandatory, we know this already, just focus on applications in class). In 2019, the EBM team decided to make the sessions non-mandatory, had fewer sessions, and only focused on applications in class. When we analyzed the EBM data for 2019, students performed $\sim 11 \%$ lower on EBM questions, students complained that they needed to discuss the basics in class, and students felt the learning objectives and goals for each session were not met. For this current academic year (2020), faculty kept all sessions mandatory, had more sessions, discussed basic concepts, and had application exercises in their sessions. The students' comments and performance indicate that they are once again happy with EBM. CONCLUSION: Utilizing student comments and feedback, while helpful, should not be the only thing that governs curriculum decision changes. Considerations should also include performance data, faculty thoughts, and content.

\section{APPLYING CONTENT ANALYSIS FOR DEVELOP- MENT OF PROFESSIONALISM CURRICULUM}

Mary Manis

Sam Houston State University—Huntsville, TX

PURPOSE: Many definitions exist for professionalism in medicine, and various expert organizations have published recommendations for professionalism curriculum requirements for undergraduate medical education. In order to determine which concepts should be included in the professionalism curriculum of a new osteopathic medical school, content analysis methods were applied to these documents. This abstract describes the project and its outcomes. 
METHODS: One year prior to the start of our fall 2020 inaugural class, quantitative content analysis was applied to assess a sample of internal programmatic educational objectives and external documents directed at professionalism in osteopathic medical students, medical residents, and practicing physicians. An initial interactive set of categories was determined, text was coded by hand, and categories were added through the coding process. Translation rules were developed from counts of the most frequently occurring words or word pairs, literature review, and personal professional knowledge. Proximity analysis was applied to determine co-occurrence of concepts, and a cognitive map was created for data visualization.

RESULTS: Content analysis yielded 6 principles of medical ethics and 43 words and two-word groups (i.e. respect and informed consent), and identified interrelationships between them that form the components of professionalism in the sample documents. The cognitive mapping and data from conceptual frequency and proximity analysis were used to outline and develop material and plan delivery of longitudinal four-year curriculum in professionalism at our osteopathic medical school.

CONCLUSION: Applying content analysis methods to "communications" from expert sources allowed a structured approach to identifying and presenting important aspects of professionalism curriculum requirements in a clear and effective manner. The results have led to the development of comprehensive curriculum materials and a plan for their cohesive delivery to our osteopathic medical students.

\section{ELEVATING THE VOICES: INCORPORATING MUL- TIPLE NARRATIVES IN THE STUDY OF DISEASE IN A GASTROINTESTINAL AND NUTRITION SEC- OND-YEAR MEDICAL COURSE}

\section{April Hatcher I Lauren Craig}

\section{University of Kentucky}

PURPOSE: Basic science coursework in the medical curriculum is traditionally focused on biological underpinnings of disease processes. There is a call, however, for a deeper dive into the sociocultural influences on disease to be threaded throughout the curriculum. This abstract describes a classroom innovation designed to incorporate multiple diverse perspectives to enrich students' understanding of the ways in which social determinants of health affect various health risks and outcomes.

METHODS: The project functioned as a virtual mini-symposium. Groups were assigned to six categories: malnutrition, oral/pharyngeal health, esophageal/gastric disease, chronic liver disease, inflammatory bowel disease, and GI cancers. There were six groups per category (5-6 students per group). This assignment was scaffolded to first include a group-selected title/description within the overarching theme and a role each student would interview. For example, one group interviewed these roles: medicine/pediatric physician, a patient's legal guardian, pediatric gastroenterologist, a special needs foster care home manager, and a registered dietician nutritionist. Students submitted 3-5 questions for their interviewee as part of the assignment.

RESULTS: For the symposium there were 36 small group presentations scheduled throughout a two-hour period via six different Zoom accounts. Groups presented a brief background of their project, a creative synthesis of the narratives each student brought back to the group, and reflections on how they would use lessons learned. Students also attended presentations of their peers; during each 20-min interval, there were six concurrent sessions presented on a range of GI conditions.

CONCLUSION: This project was designed for students to move beyond an understanding of the pathophysiology of a disease process and explore the societal and cultural factors that are integral to the lived experience of the patient. The college community supported the students. The Dean and College of Medicine leadership, including the basic science course directors, members of the curriculum committee, and associate deans attended.

\section{THE EFFECT OF DRAWING MICROBIOLOGY CON- CEPTS ON SHORT-TERM RETENTION BEFORE AND AFTER INTERRUPTED LEARNING}

\section{Robert Waters}

\section{Lake Erie College of Osteopathic Medicine}

INTRODUCTION: During preclinical education medical students learn numerous concepts that must be retained and added to over time, even when not being actively used or when learning about new unrelated concepts. Previous studies have shown that utilizing drawing as a learning tool leads to improved retention. However, limited studies have been conducted on the use of drawing activities at the medical school level. Therefore, the goal of this study was to utilize mechanism-based drawing aimed at presenting conceptual material, rather than strict memorization of facts before and after interrupted learning.

METHODS: Participants were randomly assigned to a drawing or a text group. Both groups received text \#1 detailing a microbiology concept. They were instructed to read, but only the drawing group received a drawing prompt. The groups were given 15 minutes to read, take notes, or draw. The groups completed post-test \#1. Part two of the study the groups received text \#2 with no drawing prompt. Both groups were instructed to read and take notes for 15 minutes. 
The two groups completed posttest \#2 which covered topics from text \#1 and \#2. A t-test or Mann-Whitney U was performed and $\mathrm{p}<0.05$ was considered significant.

RESULTS: The drawing group performed significantly better on post-test \#1 compared to the text group. There were no significant differences on overall performance on posttest \#2. However, the drawing group performed significantly better on questions related to the material covered in the text \#1 on post-test \#2.

CONCLUSION: Results presented here show that students perform significantly better when immediately tested on microbiology text while drawing, even after the introduction of an unrelated concept. A future study will be conducted to investigate the effectiveness of an online drawing workshop that will supplement concepts taught during lecture.

\section{STEPS ON ESTABLISHING A FACULTY DEVELOP- MENT CURRICULUM FOR HEALTH SCIENCE EDU- CATORS IN A NEW MEDICAL SCHOOL}

\section{Karina Madrigal}

\section{University of Texas Rio Grande Valley}

PURPOSE: New Medical Schools need health science educators, to teach throughout the medical education continuum from basic science to clinical years. The need to establish a defined faculty development curriculum for health science educators will aid in standardizing best teaching practices and build a potential master teacher to move the curriculum toward active learning and innovation. The UTRGV SoM is a distributed campus, to achieve increase outreach we will offer synchronous and asynchronous online faculty development during the pandemic. This poster will share our progress to date.

METHODS: A pilot, faculty development program, was established from faculty needs assessment data, teaching observations, and student evaluations was rolled out in FY 2020. A designated faculty development curriculum was recommended to faculty and provided via synchronous and asynchronous viewing through videos and PowerPoints on a designated Blackboard site. The advancement of the continuum of best teaching practices will be measured by sessions completed, faculty feedback, and comparison of prior F2F faculty development data for 2016-2019.

RESULTS: We will present how this need-based curriculum session is delivered and received for best teaching practices among health science educators. We will discuss the cost of establishing the program, return on investment, advantages, and barriers to online implementation in a distributed campus during a pandemic.

CONCLUSION: There is a need to improve faculty development opportunities that are interactive, self-directed, and offered online. These recommendations could result in increased synchronous and asynchronous faculty development attendance and learning and, in turn, increased student academic achievement.

\section{E-Learning}

TRANSITIONING TO ONLINE-ONLY LEARNING FOR MEDICAL EDUCATION DURING THE COVID-19 PANDEMIC: STUDENT AND FACULTY PERSPECTIVES

Caitlin A. Williams | Jackie T. Nguyen | Harmandip Singh | Amanda J. Chase I Johannes W. Vieweg

Dr. Kiran C. Patel College of Allopathic Medicine, Nova Southeastern University, Fort Lauderdale, FL 33328, USA

PURPOSE: The aim of this study was to assess the perceptions of both students and faculty in undergraduate medical education regarding the rapid transition to online learning during the COVID-19 pandemic. Since the unprecedented nature of this pandemic necessitated switching to a completely online environment, we aimed to investigate the feasibility and benefits of fully online learning for undergraduate medical education.

METHODS: This study was conducted in 2020. Two surveys were created, one survey for faculty and one survey for students. 73 faculty members and 104 medical students were surveyed, and their responses collected. Data was analyzed qualitatively for thematic trends and quantitatively to include frequencies and Chi-square analysis.

RESULTS: Data indicate that although the majority of students and faculty members found online learning to be convenient and accessible, they prefer in-person courses. Both groups felt the transition was adequate and appropriate institutional support was provided. However, students expressed feelings of decreased engagement and collaboration in online courses when compared to in-person courses. Faculty members also conveyed the time-consuming nature of creating and converting content to online platforms, as well as the difficulty developing relationships with the students. CONCLUSIONS: This study suggests online learning is feasible but is not the preferred method for teaching and learning by students and faculty. The transition to an online learning environment was necessary given the circumstances surrounding COVID-19. Although this development was rapid and emergent, there are many salient points that can be learned from this situation in order to better online learning in undergraduate medical education. Recommendations include increasing faculty training for online learning software and technology, identifying comprehensive resources for online learning to mitigate the need for multiple 
platforms, developing techniques to engage students more consistently, and developing spaces for online learning on campus in situations other than a global pandemic.

\section{EDUCATING THE COMMUNITY ON COVID-19 IN CHILDREN THROUGH A STUDENT-LED, BILIN- GUAL SERVICE-LEARNING PLATFORM}

Yae Kye I Cicilyn Xie | Kei Takigawa | Brenda Castillo | Jessica Chacon PhD I Niti Manglik MD

Department of Medical Education, Paul L. Foster School of Medicine, Texas Tech Health Sciences Center El Paso, El Paso, TX 79,905, USA; Department of Pathology, Paul L. Foster School of Medicine, Texas Tech Health Sciences Center El Paso, El Paso, TX 79,905, USA

PURPOSE: In response to the COVID-19 pandemic, Paul L. Foster School of Medicine created a task force, El Paso Health and Education Awareness Team, to conduct studentled virtual health fairs (VHF) on various SARS-CoV-2/ COVID-19 topics in English and Spanish. The platform's goal was to provide the community with evidence-based, timely education on COVID-19 and to allow students to actively engage with the community. For this platform, we created a presentation on the "Symptoms and preventative measures for COVID-19 in children and adults" to increase awareness of COVID-19 infection in children.

METHODS: VHF were hosted live on Zoom to the El Paso, TX communities and University of Pennsylvania premedical students. Presentations were created and delivered by students under faculty supervision. After each VHF, a 5-point Likert scale survey was sent to participants by email to assess the educational platform. In order to evaluate the effectiveness of our presentation and measure participants' knowledge, we conducted a 5-question pre-assessment and post-assessment quiz, consisting of the same questions. The percent of correct answers per question was calculated from each health fair and averaged to derive the mean. Significance was determined using proportion t-tests.

RESULTS: Post-VHF survey results were very positive with $92 \%(n=77)$ of responses agreeing that our session was useful. Up to a $37 \%$ improvement was observed in the average percent of correctly answered questions when comparing the pre-assessment $(70 \%, 29 \%, 56 \%, 87 \%$, and $36 \% ; n=63)$ and post-assessment $(80 \%, 66 \%, 88 \%, 97 \%$, and $72 \% ; n=60)$ quiz results. Significant improvement was found in 4 of the 5 questions $(\mathrm{P}<0.05)$.

CONCLUSION: Participants correctly answered the questions with better accuracy after the presentation suggesting that our presentation on COVID-19 in children was informative. We conclude that VHF have the potential to be powerful tools to teach and disseminate information to a broad audience.

\section{MEDICAL STUDENTS' PERCEPTIONS ABOUT USING AN E-LEARNING PLATFORM FOR PATHOL- OGY TUTORIALS DURING COVID-19}

Suja Pillai I Viveeka Vimalanathan I Edward Farley I Isini Muthumuni I Mei-Ting Chen I Peter Simpson I Glenda Gobe

School of Biomedical Sciences, Faculty of Medicine The University of Queensland, Brisbane, Australia I School of Clinical Medicine, Faculty of Medicine The University of Queensland, Brisbane, Australia I UQ Centre for Clinical Research

PURPOSE: Covid-19 has forced educators around the world to change their conventional face to face teaching method to a virtual mode. Prior to COVID-19, pathology tutorials in University of Queensland were delivered to Year 1 and 2 MD students via in person small group tutorials (40 students). Due to COVID-19 lockdown, all small group tutorials ceased and steps were taken to run tutorials in large groups ( $>40$ students) via Zoom (a video conferencing tool). The aim of this study is to gain quantitative and qualitative insight into the effectiveness, value and potential future utilisation of the e-learning platform for pathology tutorials. METHODS: The 2nd year MD students, who had previously completed one whole year of in person pathology teaching, were invited to complete an online questionnaire designed to evaluate their opinions about Zoom platform after the e-tutorial.

RESULTS: A total of 75 students took part in the online survey, and $>50 \%$ of students strongly agreed that switch to e-tutorials was more effective and engaging than faceto-face tutorials. Most respondents reported a significantly higher $(66 \%)$ understanding from the e-learning tutorials that improved their understanding of pathology. However, a proportion of students (38\%) expressed that online tutorials should not replace in-person tutorials but run alongside them. In regards to future tutorial delivery, the majority of respondents believed that e-learning tutorials should either replace in-person tutorials (55\%) or be conducted alongside in-person tutorials (42\%).A thematic analysis of the qualitative comments has strongly indicated that online pathology teaching was 'extremely useful', 'easier to engage' and 'better structured' compared to face-to-face teaching.

CONCLUSION: E-learning platform for pathology teaching and learning during COVID-19 resulted in a positive learning experience in pathology and medical educators should continue to engage with pathology e-learning even post-Covid-19. 
UTILIZING GOOGLE FORMS FOR VERSATILE ONLINE MEDICAL EDUCATION IN THE ERA OF THE COVID PANDEMIC

\author{
Pratima Singh, MD I Rebecca Bowden, PhD I Jozia \\ McGowan, DO
}

Kansas City University College of Medicine

PURPOSE: The COVID pandemic required immediate adoption of on-line medical education curriculum in 2020. In the fall semester of 2020, interactive pre-class student preparatory exercises and virtual small groups utilized Google Forms to guide learning and application. The variety of approaches to Google by different faculty and the resulting student experiences are presented.

METHODS: Three separate approaches to google form document instructional design via synchronous and asynchronous delivery were utilized for first and second year students. The first was pre-class student preparatory exercise wherein students learned about amyloidosis and library e-resources through an "escape room" google form. This required participants correctly answer questions about amyloidosis in order to progress to the next page. The second was an in-class small group assignment, where groups of students worked through immunology case-based presentations and entered answers to progressive questions; these responses were tracked and reviewed by teaching faculty. The third was another in-class small group exercise, with an "escape room" design. Students progressively unlocked sequential aspects to a case presentation by entering correct answers through a patient work-up.

RESULTS: Question results were easy to download and score by Immunology faculty within Google. The free response items were thoroughly answered by the majority of the respondents. Quantitative data through Menti polls for one exercise indicated $79 \%$ were satisfied with this event, $82 \%$ learned something, and $89 \%$ wanted more like it in the future. Written course evaluations for all three experiences were uniformly positive with students commenting on how the use of Google forms led to engaging interactive educational experiences.

CONCLUSION: Google forms is a highly versatile tool for interactive on-line medical education. There are numerous options for active learning that can be utilized, and the addition of a "gamification" approach gives students a refreshing learning experience that was viewed as fun and engaging.

MAINTAINING TRANSFORMATIVE GLOBAL MEDICAL STUDENT ROTATIONS: INNOVATIVE USE OF WHATSAPP WITH TELEMEDICINE
Elizabeth S. Rose, MPH MEd I Eiman Jahangir, MD MPH । Marie H. Martin, PhD Med

Vanderbilt Institute for Global Health, Vanderbilt University Medical Center, Nashville, TN, 37,203, USA I Division of Cardiovascular Medicine, Department of Medicine, Vanderbilt University Medical Center, Nashville, TN, 37,203, USA

PURPOSE: Through a global health clinical immersion course, students at a medical school in the United States of America (USA) have engaged with clinicians and patients across the globe in low-resourced settings, gaining valuable perspective about populations, cultures, and diseases. After travel was suspended due to the COVID-19 Pandemic, alternative means to engage in clinical activities was needed.

METHODS: Students engage in month-long international clinical rotations in low-resourced settings. Pre-departure training coupled with didactic digital modules and distance mentoring sessions during the course introduce students to topics of cultural competency, global health ethics, and local clinical and public health interventions. In 2020, instructors pivoted the clinical experience to telemedicine consults. They partnered with a non-profit organization based in the USA that utilizes telemedicine to connect frontline health workers in medically underserved areas of the world with volunteer medical specialists. A student piloted this telemedicine course in partnership with a Kenyan health team. The Kenyan clinicians and student communicated through WhatsApp and the organization's telemedicine platform. WhatsApp provided tele-video interactions during patient visits.

RESULTS: Student feedback during the five years of the in-person course indicated that this multi-modal learning experience exposed students to different perspectives about medicine, changing their clinical practice. To maintain this transformative experience, the use of an asynchronous platform with synchronous video conferencing was successful in allowing the student to meaningfully engage with Kenyan clinicians. Through this hybrid telemedicine format, the student worked with clinicians across Kenya and was exposed to multiple region-specific disease burdens and cultures. WhatsApp enabled the student to interact with clinicians and patients, converting a previously asynchronous patient care platform into a dynamic experience. The Kenyan clinicians viewed the student as a vital team member.

CONCLUSIONS: Student engagement in telemedicine consults supported by WhatsApp can be used successfully with international partners and clinicians when physical presence is unfeasible. 
MEDICAL STUDENTS' EXPERIENCE WITH AN ONLINE SARS-COV-2/COVID-19 ELECTIVE

Houriya Ayoubieh I Jorge Cervantes I Cynthia Perry I Curt Pfarr I Jessica Chacon

Texas Tech University Health Sciences Center El Paso, Paul L. Foster School of Medicine, El Paso, TX 79905, USA

PURPOSE: To reduce SARS-CoV-2 transmission, Texas Tech University Health Sciences Center El Paso-Paul L. Foster School of Medicine (TTUHSC-PLFSOM) incorporated supplementary online learning for 4th year medical students unable to complete clerkships due to clinical site restrictions. We developed an online elective: "COVID-19: Utilizing basic sciences to implement what you need to know as a physician."

METHODS: The online SARS-CoV-2/COVID-19 elective consisted of an interactive discussion board, and a student presentation to faculty and peers with a question and answer session. Students addressed 6 topics: 1) Clinical features; 2) At risk populations; 3) Modalities for detection; 4) Management plan for infected patients; 5) Applying current approach for vaccines to a COVID-19 vaccine; 6) Novel COVID-19 therapies. The educational platform was evaluated using a post-assessment survey emailed to students who completed the elective. Likert scales were used to measure satisfaction and agreement with elective benefits. Additional open-ended questions assessed the overall impact students perceived.

RESULTS: Students who completed the elective strongly agreed (60\%)/agreed (40\%) the elective was useful for learning about the SARS-CoV-2/COVID-19 and felt more knowledgeable about COVID-19 clinical features. Seventy percent felt more knowledgeable about the SARS-CoV-2/COVID-19 at-risk populations and $90 \%$ agreed that they could explain why some people were more at risk than others. Ninety percent found the elective well organized and $80 \%$ found the course engaging.

CONCLUSION: The rapid and extensive spread of the COVID-19 pandemic is a major cause of concern for the healthcare profession. Information about the novel virus, disease manifestations and management strategies are in constant flux. This is the first online elective course developed at TTUHSC PLFSOM that aims to increase student knowledge of COVID-19.This elective is designed to enable students to further their understanding of COVID-19 through directed self-study prompts that require students to research the latest scientific literature.

DESIGN AND IMPLEMENTATION OF AN ONLINE EDUCATIONAL WEBSITE FOR INTERNAL MEDICINE RESIDENTS
Shane Murray I Ana B Arevalo I Bertin Salguero I Caroline Dooley I David Weininger Cohen I John Andrilli

Mount Sinai Morningside-West Hospital Center, Icahn School of Medicine at Mount Sinai, New York, NY, USA

PURPOSE: Our residency program recently transitioned from a daily noon conference to a weekly academic halfday curriculum during the pandemic. With the academic half-day taking place almost entirely in a virtual setting, as a result of the COVID-19 pandemic, we created an online educational website for our residents. This abstract describes the project and its outcomes.

METHODS: Our website was created using Google Sites. Academic-half day sessions were recorded using the Zoom app and uploaded to the site. Residents also had access to New England Journal of Medicine (NEJM) Resident 360 content. Hyperlinks to important journal reviews, landmark trials and guidelines from NEJM were included with each session video. Content from our EKG workshops, Jeopardy sessions and afternoon reports was also included in separate sections of the website. We monitored resident engagement with the website using Google Analytics tool. Data was collected from September 1ST to December 7th 2020.

RESULTS: A total of 1,183 users visited the website. 257 (21.7\%) users were returning visitors while 926 (78.3\%) were new visitors. A total of 3,976 pageviews occurred with an average of 2.39 pageviews during a session. The intern inpatient survival guide had the highest number of individual pageviews at 396. The academic half-day and EKG workshop pages were viewed 82 and 50 times respectfully. $499(53.8 \%)$ new users visited the site using a mobile device while 427 (46.2\%) used a desktop or laptop.

CONCLUSION: Educational websites are an important resource that can be serve as an educational hub for residents and improve engagement with educational activities.

\section{EBITE: A MEDICAL EDUCATION BLOG FOR A COMMUNITY OF LEARNERS}

\section{Elisabeth Schlegel}

\section{Zucker School of Medicine}

PURPOSE: The disruptions in medical education and faculty development delivery due to the public health concerns during the Spring of 2020 rapidly led to re-establishing educational discourse through web-based tools including text-driven media such as blogging. Specifically, educational blogs can support learning comparable to online discussion forums with the added benefit of attractive content presentation using text, images, audio and video, appealing to a variety of learning preferences. Contextualized by 
sociocultural and constructivist theories, a community of learners establishes rapidly as readers engage with the content and exchange reflections and opinions in the comment sections. We developed the educational blog eBite to deliver content aligned with corresponding faculty development sessions. eBite is easy to access from anywhere on any device and enhances learning and reflection of target audiences at their own pace as well as interested readers worldwide.

METHODS: eBite blog entries are posted by the medical education expert either prior or after a corresponding faculty development session to enhance and review concepts discussed. Satisfaction, collaboration, exchange of ideas and ease of use of eBite was examined using a mixed-method approach using a 5-point Likert-type survey including narrative questions to obtain additional insights. Survey data and themes will be presented.

RESULTS: Initial data indicated benefit through (1) mental accessibility of complex topics, (2) stimulating critical thinking, and (3) reflection on in-person-sessions supported by eBite content. Additional value is establishing instant rapport with the audience, as eBite is introduced in the initial email preceding an upcoming session.

CONCLUSION: The educational blog eBite is an effective tool promoting deep learning to continue and advance the educational discourse of faculty development. Using multimedia display options and blog functionality, a growing community of learners/educators of all levels can connect with each other, expanding each other's horizon.

\section{SIMILAR EDUCATIONAL OUTCOMES FOR VIR- TUAL VS CLINICAL TRACKS IN A FOURTH YEAR GERIATRIC PHARMACOTHERAPY ELECTIVE}

\section{Laurel Gorman I Andrea Berry | Mariana Dangiolo}

\section{UCF College of Medicine}

PURPOSE: Given prescription errors contribute to geriatric morbidity and mortality, many medical education institutions have sought to improve approaches to geriatric pharmacotherapy undergraduate medical education. At our institution, a fourth year (M4) geriatric pharmacology elective was developed using innovative interactive virtual educational tools with differing tracks allowing learners to customize their experience. All participants completed structured virtual processes, but " $C$ " track participants shadowed an outpatient geriatrician while the "A" track researched a relevant geriatric topic virtually and produced an academic product. The goal of this pilot study was to compare outcomes for different tracks to determine if learning and perceptual outcomes varied.

METHODS: After completing required virtual activities, learners experienced the $\mathrm{C}$ or $\mathrm{A}$ tracks, then completed a geriatric knowledge quiz with multiple choice questions (MCQ) and a 1-5 Likert survey (where 5= strongly agree to $1=$ strongly disagree) rating themes of clinical relevance, resource utilization, polypharmacy awareness, confidence, and impact on future practice.

RESULTS: Geriatric pharmacology knowledge increased for all learners taking the elective with a pre-experience score $=68+17 \%$ vs post-elective score $=92+11 \%$ (mean $+\mathrm{SD} ; \mathrm{N}=19 ; \mathrm{p}<0.01$, paired T-test), The post-quiz difference between $\mathrm{C}(93+10 \% ; \mathrm{N}=6)$ and $\mathrm{A}(91+11 \%$; $\mathrm{N}=13$ ) was not significant $(\mathrm{p}=0.6$, $\mathrm{T}$-test $)$. Learners rated both experiences highly (mean Likert ranges 4.5-4.8; $92-100 \%$ affirmative) in all theme categories with no significant differences detected by T-tests between means for $\mathrm{C}$ and $\mathrm{A}$ tracks.

CONCLUSIONS: Preliminary data support that learners valued both tracks of the geriatric elective. Exposure to either option was associated with increased geriatric pharmacology knowledge and overwhelmingly positive perceptions of educational value, although $\mathrm{C}$ track learners tended to rate relevance slightly more highly. However, many M4 learners traveled for interviewing and valued the flexibility of the virtual A track as well as the opportunity for deeper learning associated with researching complex geriatric pharmacology topics.

\section{EFFECT OF VIDEO CONFERENCING ON STU- DENT ACADEMIC PERFORMANCE: EVIDENCE FROM PRE-CLINICAL SUMMATIVE ASSESSMENT SCORES}

Andrew Darr I Jenna Regan I Yerko Berrocal

University of Illinois College of Medicine Peoria

PURPOSE: Medical education has rapidly transitioned to remote delivery to support social distancing during the COVID-19 pandemic. Anecdotal evidence indicates that learners experience increased fatigue and burnout from multiple hours on virtual platforms, colloquially termed "Zoom fatigue". However, there is scant data to indicate whether remote delivery impacts learning outcomes in medical education. We report a comparison of assessment outcomes from an undergraduate medical education course pre- and post-transition to remote delivery.

METHODS: Performance data from summative assessment of second year medical students at the University of Illinois College of Medicine Peoria for the Brain \& Behavior course was compared for consecutive years (2019 and 2020). In 2020, the entire course was delivered remotely, with all interactive sessions (47\% of total content) occurring synchronously over ZoomTM. In contrast, all interactive synchronous sessions in 2019 were in-person. Assessment items 
with a discrimination value of 0.2 or greater were categorized according to content delivery method and analyzed using Student's t test to compare mean scores from 2019 and 2020.

RESULTS: Overall assessment scores were comparable between 2019 and 2020. However, items which assessed interactive, synchronous content in 2020 (remote) had mean scores that were significantly lower than 2019 (in-person) by 13 points, $\mathrm{t}(44)=2.54, \mathrm{p}<0.05$. Interestingly, summative exam performance in the preceding course (the first in 2020 to transition entirely to remote learning) showed no appreciable difference between years.

CONCLUSION: High value is placed on synchronous learning, during which learners have the opportunity to interact with faculty and peers. These data offer evidence that, over time, the efficacy of synchronous learning via virtual platforms declines compared to in-person activities. Factors that may contribute to lower assessment performance include learner fatigue and social disconnection when interactive, synchronous content is delivered through a virtual platform.

\section{FAIR VIRTUAL MEDICAL EDUCATION DURING THE COVID-19}

\section{Shima Tabatabai}

Shahid Beheshti University Of Medical Sciences, Tehran, Iran

BACKGROUND \& PURPOSE: The COVID-19 pandemic has a critical impact on Medical education. The medical sciences universities have developed strategies for increasing the student's safety by shifting to online learning to keep medical education on track. Adapting to the pandemic is necessary to keep fair medical sciences education on stream across the country at this unprecedented time. This study aimed to address this question: If schools continue virtual learning, what are possible strategies for fair and successful medical education?

METHODS: In this applied qualitative research, using purposive sampling, we select seven experts and key decisionmakers in Iran's medical education system to participate in a panel discussion. The data was analyzed by thematic framework approach.

RESULTS: Recommendations to enhance equity in Academic Learning throughout the COVID-19 were presented in 5 main themes. Ensure fair and effective clinical training. Developing a national partnership between medical schools to provide standardized educational content. Regarding the importance of integrated and fair medical education across the country, The IRAN'S Virtual University of Medical Sciences (VUMS) in partnerships with leading Iranian medical schools is providing MOOCs, accessible free of charge.
Also, the educational contents deliver through the National LMS to offer integrated learning experiences. Using available collaboration tools such as Zoom, Skype, and Google Hangouts Meet. Using formative assessments to provide constructive, effective feedback to medical students as learning tools. Developing educational strategies for maintaining, and strengthening learning and teaching collaborations in the virtual environment to prepare future clinicians for practice. Medical sciences educators and Clinical mentors will need to build a new skill set in order to adapt to the new situation.

CONCLUSION: A key challenge in the transition to virtual medical education is to address educational inequities at this unprecedented timeThis article discussed the recommended strategies for fair and integrated learning in the face of the pandemic.

\section{STUDENT LED DESIGN OF A PRE-MATRICULA- TION PROGRAM FOR MEDICAL STUDENTS}

Jieun Lee, M.S. I Romany Harkas I Stephanie Pearson I Kyle Bauckman, $\mathrm{PhD}$

\section{Dr. Kiran C. Patel College of Allopathic Medicine}

PURPOSE: This study aims to provide a systemic view of medical school pre-matriculation programs (PMPs) and apply that data alongside Nova Southeastern University Kiran C. Patel College of Allopathic Medicine (NSU MD) faculty and student perspectives to design a PMP best fit to the needs of incoming NSU MD students. PMPs are increasingly being implemented by medical schools to ease transition of incoming students but are widely variable. In the literature, there is scarce information on how these programs currently exist systemically throughout different medical schools. As such, we have identified a need for a systematic view of PMPs, especially considering recent events such as the COVID-19 pandemic and transitioning of USMLE Step 1 to pass/fail. We have also recognized the potential of NSU MD's PMP to become more effective at transitioning incoming students.

METHODS: A survey was created targeting all United States medical schools to gain perspective on how schools are utilizing PMPs. Four additional surveys were created to understand the perspectives of NSU MD faculty and students regarding PMPs. We have also used previous feedback and personal experiences as post-didactic students to jumpstart design of the new PMP.

RESULTS: Based on survey data, we developed a selfguided curriculum by creating scaled learning objectives relative to matriculant counterparts. The entire course is asynchronous and delivered online in order to increase 
access to all students. Key topics were selected based on student and faculty feedback.

CONCLUSION: PMPs are an increasingly popular tool of medical schools that we are exploring. We have created five surveys to help accomplish the goals of this ongoing project: contribute to medical education knowledge regarding PMPs, create a system to identify students for the NSU MD PMP, and design a new PMP that best prepares incoming students for NSU MD.

\section{MAPPING METABOLISM: A NEW INTERACTIVE LEARNING TOOL FOR BIOCHEMISTRY}

\author{
Raj Joshi I Megha Verma | Bella Mauro I Harrison Kronfeld
} I Dr. Judith Binstock

TouroCOM-Harlem

PURPOSE: Stanford's Metabolic Map has been used to teach biochemistry to many medical students. While the map displays high yield pathways and enzymatic function, it does not include the specific diseases, treatments, and genetics associated with these pathways. Students have always learned to incorporate this material through individual classes throughout their initial preclinical years, without a concise, compiled document for metabolic diseases. We have created a learning tool that incorporates all these elements into a single interactive tool. This abstract describes said learning tool.

METHODS: Our program runs via Prezi, an online presentation tool. It is interactive in nature, allowing students to search and freely explore the entirety of the metabolic map at their disposal. Mapping Metabolism is organized via enzymatic pathways using different subtopic "bubbles" under the following categories: Name of the enzyme associated with a Genetic Disease: "Disease"-Clinical Presentation "Function"-Function of the enzyme and its reaction/ regulation "Genetics" - Name of the Genetic Disease/ inheritance pattern and OMIM number "Treatment"-Known treatments, e.g. enzyme replacements, gene therapy, medications "Mnemonic"-Fun mnemonics for memory Reference bubble within each bubble In addition, each pathway has a review "Quiz" bubble to help emphasize the main points of the genetic diseases in the pathway.

RESULTS: The content of Mapping Metabolism is derived from reputable medical resources that includes, but is not limited to the following: UpToDate, DynaMed, National Organization of Rare Disorders (NORD), etc.

CONCLUSION: The students and faculty of TouroCOM have been working together to combine innovative technology with the most up to date medical resources, to create a new and succinct tool to study biochemistry. Our final product will be a transformative platform that students across the country can use for their board studies and beyond.

\section{STUDENT PERFORMANCE ON NEUROANATOMY TEST QUESTIONS UNAFFECTED BY DIGITAL LEARNING MODALITY}

\section{Pallavi Juneja}

Wake Forest University School of Medicine

PURPOSE: COVID-19 has undoubtedly accelerated technological innovation in medical education. Literature shows that medical students have embraced the online, synchronized model. This study focuses specifically on the utility of synchronous virtual learning in comparison to in-person learning for the complex topic of neuroanatomy.

METHODS: All first-year medical students taking neuroanatomy were given a 10 -question pre-test and invited to participate in a one-hour review session. Of those who participated, some attended live and others streamed the session synchronously. All were given a 10-question post-test. The post-test scores and vascular neurology question performance were compared between the two groups.

RESULTS: There was no significant difference in gender, age, or neurology background between the in-person attendees and synchronous virtual attendees. Overall, students who participated in the review session synchronously virtually performed neither better nor worse on the post-test or the vascular neurology final exam questions than those who attended live. However, within the synchronous virtual learning group, men performed 9.4 points lower than women $(\mathrm{p}=0.016)$.

CONCLUSION: Overall, synchronous virtual review of neuroanatomy appears to be an acceptable alternative to live sessions with regard to student performance on neurology test questions. However, men are less successful synchronous virtual learners than women, perhaps because they are more distracted in the virtual environment.

\section{Instructional Methods}

\section{STUDENT EVALUATION OF ASYNCHRONOUS LEC- TURES FOR CORE MEDICAL SCHOOL COURSES}

Tracy Tylee I Bruce Silverstein

University of Washington

PURPOSE: With the onset of the COVID epidemic in 2020, medical school courses at University of Washington 
transitioned to an online format. We developed pre-recorded asynchronous lectures to replace in-class lectures and evaluated the students' response to this change.

METHODS: Lectures were given via Power Point and recorded using desktop recording software, then uploaded to the course Canvas page. Students had access to all lectures for the duration of the course. At the end of the course, students completed an evaluation including feedback on the pre-recorded lectures.

RESULTS: Of students who completed the end of block survey ( $\mathrm{n}=57,54 \%$ response rate), $86 \%$ rated the pre-recorded lectures as very good or excellent, $86.5 \%$ preferred prerecorded to live lectures. Reasons for preferring the prerecorded lectures included: able to watch on their own time, ability to pausing videos and review topics, efficient way to convey the material. They preferred shorter videos and felt longer lectures were more confusing and found it harder to maintain focus. Final grades were similar to previous years (87.9\% in 2020 vs $87.7 \%$ in 2019).

CONCLUSIONS: Students overwhelming preferred the prerecorded lectures and felt it gave them more control over their learning experience. Our lectures were not optimized for online presentation, ranging in length from $25-50 \mathrm{~min}$, which students noted was too long. In the future, we plan to redesign the lectures to be shorter in length, dividing a single lecture into 2 or 3 focused topics. Studies have found that students find value in pre-recorded online lectures when compared to in-person live lectures and our experience supports this finding. Give the positive response, we plan to continue to use pre-recorded lectures for the large group portion of our course, even when able to return to in person teaching.

\section{A LOOK INTO MEDICAL STUDENTS' KNOWL- EDGE ON ADVERSE CHILDHOOD EXPERIENCES}

\section{Lara Fawaz I Nelia Afonso, MD}

Oakland University William Beaumont School of Medicine

PURPOSE: Adverse childhood experiences (ACEs) are defined by the Center for Disease Control and Prevention as traumatic events that occur from birth to 17 years old that involve abuse, neglect, and household violence. Literature has shown that ACEs are linked to various mental and physical health consequences, thus it is imperative that healthcare providers are given early education on how to identify and care for these patients. Currently, training on ACEs is lacking in most medical school curricula. The aims of this study were to assess medical student knowledge of ACEs and confidence in caring for patients with them.
METHODS: Students at a midwestern allopathic medical school were asked to complete an anonymous survey on their knowledge and experiences with ACEs.

RESULTS: 83 out of 494 (16.8\%) completed the survey. Although $68 \%$ of students were able to correctly determine what ACE stands for, only $13 \%$ were able to correctly identify situations that constitute an ACE. While most identified scenarios dealing with physical abuse/neglect as ACEs, emotional stressors were missed by $77 \%$ of students. $45 \%$ had experiences with ACEs in the clinical setting. Only $22 \%$ of students considered themselves confident in screening patients for ACEs, and only $21 \%$ of students felt confident in treating patients with ACEs. There was no statistical significance with any variables in the study with time periods of exposure (all $\mathrm{P}>0.05$ ).

CONCLUSION: This study identified a lack of knowledge and confidence among students in regards to what ACEs are, what types of scenarios constitute ACEs, and how to treat patients with them. With the overwhelming evidence of future health complications associated with ACEs, it is of paramount importance for future physicians be taught to screen and care for patients with a history of ACEs. This study will encourage medical educators to incorporate teachings about ACEs early on in medical school.

\section{INVESTIGATION OF A CLINICAL MICROBIOL- OGY AND IMMUNOLOGY COURSE ON PERCEP- TIONS AND PERFORMANCE IN THE FIRST YEAR OF OPTOMETRY SCHOOL}

Joshua Costin, PhD I Michelle Demory Beckler, PhD I Beata Lewandowska, OD

NOVA Southeastern University

PURPOSE: Of the 23 optometry programs in the USA, all require undergraduate microbiology as a pre-requisite to enter their program, yet NSU is one of only 11 optometry schools in the country that require its students take a medical microbiology course. Worse, immunology is not a pre-requisite for entry into optometry schools and NSU is one of only 9 programs that require medical immunology as part of its curriculum. Basic knowledge of microbiology and immunology is part of the licensing exam optometrists must pass in order to practice -information that is needed in order to diagnose patients in practice and understand how to protect themselves, their staff, and their patients from infections commonly spread in optometry practices. In spite of all of this, many students fail to see the relevance of microbiology and immunology to their chosen profession, particularly in light of its lack of emphasis in most optometry programs. 
METHODS: In the Fall of 2019, the microbiology and immunology course was significantly altered to include more of a focus on ocular manifestations of infections and immune responses and clinical lectures delivered by certified optometrists were introduced into the course for the first time. In Fall 2020, students were voluntarily surveyed online throughout the course for their attitudes towards the material and the class after the clinical lectures. Performance in the class was measured via examinations.

RESULTS: Students have thus far voluntarily returned the surveys in large numbers. Anecdotally, there is noticeable excitement and increased engagement from students after each clinical lecture.

CONCLUSIONS: Since until very recently there have been a general lack of optometry programs that offer microbiology and immunology to its students in any form, there is a gap in information and understanding related to the influence and effectiveness of offering medical microbiology as well as clinically-based presentation of material.

\section{USING COMMON CLINICAL PROCEDURES TO TEACH ANATOMY IN MEDICAL SCHOOL}

Brenda Castillo* | Amanda Tran* | Brian Jacinto | Dolgor Baatar, M.D., Ph.D. I Ricardo Belmares, Ph.D. I Thomas Gest, Ph.D. I Heather A. Balsiger, M.S.

Department of Medical Education, Paul L. Foster School of Medicine, Texas Tech Health Sciences Center El Paso, El Paso, TX 79905, USA

*These authors contributed equally to this work.

PURPOSE: Medical student confidence to apply anatomy knowledge in clinical practice has been shown to be low. However, the integration of clinical aspects with anatomy improved their confidence in the subject. In our study, we introduced the teaching of common clinical procedures into the anatomy laboratory sessions to assess if this approach enhances the acquisition of anatomy knowledge.

METHODS: Four dissections were performed by the research staff to teach the clinical procedure and related anatomy of the following: femoral artery puncture, spinal tap, radial artery puncture, and subclavian vein catheterization. Each demonstration had a pre- and post-test of 1 anatomy question relevant to the clinical procedure being performed. Results were evaluated using a Paired T-test.

RESULTS: The post-test (P2) values were significantly higher than the pre-test (P1) values for all procedures. Femoral artery puncture: $\mathrm{P} 1=22.5 \%, \mathrm{P} 2=45.0 \%, \mathrm{p}<.0001,95 \%$ CI $[-0.284,-0.171]$. Spinal tap: $\mathrm{P} 1=34.6 \%, \mathrm{P} 2=77.6 \%$, $\mathrm{p}<.0001,95 \%$ CI $[-0.54,-0.32]$. Radial artery puncture $\mathrm{P} 1=35.71 \%, \mathrm{P} 2=82.65 \%, \mathrm{p}<.0001,95 \%$ CI $[-0.554$,
-0.394]. Subclavian vein catheterization: $\mathrm{P} 1=12.9 \%$, $\mathrm{P} 2=76.88 \%, \mathrm{p}<.0001,95 \%$ CI $[-0.741,-0.547]$.

CONCLUSION: The study suggests that teaching anatomy in the context of clinical procedures is an effective teaching method. Whether the introduction of teaching clinical procedures into the anatomy program improves student performance on anatomy portions of standard exams and whether it prepares better medical students for clinical clerkships and residencies remain to be elucidated.

\section{TEACHING IMMUNOLOGY USING THE HISTORI- CAL MEDICAL LITERATURE}

Noyan Ramazani I Edward Simanton I Neil Haycocks

\section{UNLV School of Medicine}

PURPOSE: Immunology is a perennially difficult subject for both teachers and learners. The purpose of this innovation is to enhance student engagement with the topic using a casepresentation format that is organized around analyzing early descriptions of key immunologic phenomena. Case presentations have long been a cornerstone of teaching the basic and clinical sciences. The older medical literature, which is largely observational, contains a multitude of case reports that are often explainable with a current understanding of immunology and mechanisms of disease.

METHODS: PubMed was searched for the earliest available reports of selected topics, such as chronic granulomatous disease, transferrable hypersensitivity through blood transfusion, RAG1/RAG2 deficiency (Omenn syndrome), and IgA deficiency. Case studies were created using the historical reports, which were distributed to students and used as the basis for six hours of in-class instruction. Students played the role of "detectives" and were led through a series both focused and open-ended questions to examine each case through the lens of modern medical knowledge and deductive reasoning.

OUTCOMES: The class average on the immunology exam was $81.7 \%$, which was higher than the NBME benchmark of $78.0 \%$ for those items. Anecdotal feedback from students was positive, highlighting that the historical approach was more interesting and clinical than they anticipated. Formal course survey data indicated the primary immunology instructor received high marks (4.56/5.00) with an unusual paucity of commentary (positive or negative) directed at the immunology component of the course.

CONCLUSION: Reexamining seminal observations in the field of immunology is an engaging and potentially valuable way to approach the subject. The perspectives of novice learners today and physicians from decades past bear some similarity, and the alignment of these perspectives presents 
an opportunity to enhance student interest and highlight the clinical relevance of basic immunology.

\section{CARTOONING AS AN EFFECTIVE TOOL FOR TEACHING SCIENTIFIC THINKING}

\author{
Aimee Pugh-Bernard I Scott Thompson \\ University of Colorado Anschutz Medical Campus
}

PURPOSE: Medical education and scientific thinking often involve understanding complex experimental methods. We utilized cartooning as an active learning method for understanding complex experimental methods from a diverse array of scientific articles in an undergraduate scientific thinking course. Here we describe our implementation of cartooning and how it can be used as tool to enhance clarity, instruction, and analysis.

METHODS: A cartoon is a drawing or series of drawings used to illustrate an action or subject. In our implementation as a teaching tool, students created cartoons of experimental methods from assigned readings using a digital platform to visualize their understanding of the experimental procedures. The digital nature of this approach enables students to dynamically edit and rearrange illustrated elements throughout a class period to incorporate new ideas and understanding gained from discussion as well as easily share their creations with the class. Students created cartoons on experimental procedures including the facial recognition ability of infants and the underlying motivation of animal behavior. RESULTS: Cartoons created on the same experimental method by different individuals are rarely identical, thus exposing the entire class to a diversity of understanding. When students produce cartoons demonstrating differences in experimental methods, the flexible nature of cartooning enables editing or the addition of details to improve the accuracy of the illustrated procedure. Additionally, over the course of the semester the level of detail and clarity of the cartoons evolves thus serving as qualitative assessment of student understanding of experimental procedures throughout the course.

CONCLUSION: Cartooning enables students to visualize their understanding of complex scientific methods and to think critically about the experimental design process. Visualization of how students represent experimental methods reinforces the deeply thoughtful and creative nature of experimental design as well as facilitates the assessment of student growth and evolving comprehension of the scientific method.

CONTRIBUTION OF A STUDENT-CREATED, FACULTY-VETTED, AND BIOCHEMISTRY-ORIENTED ANKI DECK TO STUDENT PREPAREDNESS AND
WELLNESS IN A PASS-FAIL MEDICAL SCHOOL BLOCK

Daniel R. Webster I Habib Abla

Texas Tech University Health Sciences Center-Lubbock

PURPOSE: Because COVID-19 has affected both the mode of delivery of essential concepts by educators and the ability of students to digest and retain them, we developed an Anki deck customized to a portion of our specific curriculum. The deck was built using the previous year's faculty-developed instructional material. We wished to discern if such a resource would both aid in student retention of essential concepts as well as provide an improved student attitude toward the block.

METHODS: Both medical and Masters students were asked if they thought a customized Anki deck would be helpful. Because of the overwhelming positive response (88.6\%) the Anki deck was constructed. A 10-question quiz was delivered both at the beginning and at the end of the unit to gauge student performance. Finally, students were administered a survey at the end of the unit that asked the degree to which the deck was used, its usefulness in preparing for the unit exam, and other questions related to student anxiety and engagement with the material.

RESULTS: Ninety-five percent of respondents reported using the Anki deck. Over 50\% reported that it contributed over $50 \%$ of their study time. Seventy-six percent reported that it was either Very Helpful or Extremely Helpful in studying for the exam, $81 \%$ percent replied that the deck increased their engagement with the material and $64 \%$ responded that the deck reduced their anxiety throughout the unit. Performance on the Anki deck post-quiz was significantly higher for the Anki users vs. the non-users $(\mathrm{p}=0.039)$, using a two-sample $\mathrm{t}$-test.

CONCLUSIONS: Maintaining academic performance while addressing student wellness during this medical crisis is challenging. The use of this deck served, in part, to overcome these challenges and may provide a critical supplementary resource for students in future years.

\section{EXAMINING THE EFFICACY OF A CUMULATIVE STUDY DOCUMENT THAT ENCOMPASSES NECES- SARY RESOURCES-WORK IN PROGRESS}

Ali Arafat I Kori Brewer, Ph.D.

Brody School of Medicine at East Carolina University I East Carolina University Department of Physiology

PURPOSE: The typical medical school curriculum requires students to reference multiple resources to learn and master 
their course material. Searching for the best resources can be counterproductive, since this time could be used to study the material instead, thus making it difficult for students to effectively understand the material and succeed on class examinations. This study will seek to determine if consolidating the most used resources for a course into one comprehensive study document will help students master the material more efficiently.

METHODS: A comprehensive study document was made available to the medical student classes of 2023 and 2024 at the Brody School of Medicine (BSOM) prior to their corresponding lectures for the brainstem topics of the Medical Neuroscience course. After the students take the comprehensive course exam, they will complete a survey assessing the document and their experience using it. The de-identified grades of students who used the document will be compared to the grades of students who did not use the document, and a comparison will be made using a paired t-test to determine if using the document had an impact on course performance. RESULTS: This study is a work-in-progress and has yet to analyze results from both classes since the class of 2024 has yet to take the exam. Preliminary data from the class of 2023 has shown no significant relationship between exam scores and use of the document, however, qualitative analysis based on the surveys is favorable towards use of the document. The final results will include the average scores of the students of both classes as well as the survey responses to assess the qualitative effect of the document.

CONCLUSION: There is potential to use the concept of a comprehensive study document across courses to minimize time spent gathering resources and improve student performance.

\section{INTERPROFESSIONAL CASE BASED LEARNING- SEQUENTIAL DISCLOSURE ACTIVITY TO PRO- MOTE CULTURAL COMPETENCY AND INCLU- SIVE HEALTHCARE}

\section{Samiksha Prasad I Chasity O'Malley I Arkene Levy I Daniel Griffin I Rolando De Leon}

\section{Nova Southeastern University}

PURPOSE: Lesbian, Gay, Bi-sexual, Transgender, Questioning, and Intersex $(\mathrm{LGBTQI}+)$ patients often experience discrimination within health care settings due to a lack of provider knowledge and biases of healthcare workers towards this population. There is a greater need to provide culturally competent and unbiased healthcare in all health professions, which can be accomplished through Interprofessional Education (IPE). With the goal of facilitating interprofessional teamwork and collaboration, an Interprofessional-Case Based Learning "Sequential Disclosure
Activity (IP-CBL-SDA) focusing on the LGBTQI + community highlights the negative impact of stigma/bias while also promoting competency in interprofessional practice will be implemented.

METHODS: An IP-CBL-SDA will be implemented for the eight health disciplines healthcare professional students at Nova Southeastern University. The students from numerous health disciplines will be placed into groups of 8-12 students to discuss roles, team goals, and outcomes for the IP-CBL-SDA. Clinical and basic science faculty from different disciplines will circulate throughout the different rooms for separate sessions and facilitate the discussion in adequate depth and appropriateness. The activity will be recurrently run throughout the single-day event (NSU IPE Day, February 26th, 2021) for eight individual 1-h sessions. Two validated instruments, the Readiness for Interprofessional Learning Scale and Health Disparities Attitudes and Knowledge Scale will be administered pre/post-session.

RESULTS: It is anticipated that $\sim 800$ students across disciplines will participate in this activity. We will analyze whether that the post-test scores reflect an improvement in the students' understanding of the negative impact of stigma and bias on LGBTQI + healthcare and an increased interest in collaborating with students from other health professions. CONCLUSIONS: This activity is expected to improve students' knowledge and attitudes towards LGBTQI+ healthcare and promote continued interprofessional collaboration towards this goal. This activity design could be applicable to other underserved populations.

\section{PERCEPTIONS OF BARRIERS AND BENEFITS OF SERVICE-LEARNING IN ALLIED HEALTH TRAINING}

\section{Neena A. Xavier, MD, FACE I Pete Jones, PhD}

Brenau University, Department of Physician Assistant Studies I University of Alabama at Birmingham, Department of Political Science and Public Administration

PURPOSE: It has been shown that homelessness is associated with poorer health status. We developed and implemented a service-learning (SL) project to improve students' ability to recognize and address social determinants of health in their medical-decision-making for this vulnerable population. This abstract describes the pilot project and changes in students' perceptions to the benefits and barriers of SL as a pedagogical tool.

METHODS: Our SL project involves a partnership with the Firehouse Shelter, a male homeless shelter in Birmingham, AL. The University of Alabama at Birmingham formed an interprofessional, student-run clinic that provided free wellness screenings to those that accessed the resources of the 
shelter. The clinic provides in-house access to diagnostic labs, screening for chronic diseases, mobility testing, and vision screening, In addition, the wellness clinic was deliberately incorporated as an SL experience in the clinical medicine course series of the physician assistant (PA) program. In the first phase of implementation of this project, we used pre- and post-questionnaires to determine student perceptions of the barriers and benefits of SL.

RESULTS: Eighty students participated in the pilot project. Student perceptions of both the benefits and barriers decreased after participating in the experience. Female students and those who had previous volunteer experience were more likely to rate benefits as higher. Their perceived barriers decreased more significantly after intentional implementation of the SL activity. Students reported a more realistic perspective of their impact on community health through a SL experience.

CONCLUSIONS: SL provides allied health programs a chance for real-world field experiences with opportunities for structured feedback to help students challenge and/or restructure conceptual frameworks through which they process information early in the didactic curriculum. We will present strategies for successful implementation of SL that can transfer to many other graduate-level programs still in their infancy with implementing SL experiences.

\section{COLLABORATION AMONG BASIC SCIENCE AND CLINICIAN EDUCATORS IN CREATING AND IMPLEMENTING HIGH FIDELITY SIMULATION SESSIONS IN AN INTEGRATED PRECLINICAL MEDICAL EDUCATION CURRICULUM}

\section{Casey N. Bassett I Aimee T. Martin I Sarah Gibbs I Matt A. Boegehold I Julie K. Gaines}

\section{Augusta University-University of Georgia Medical} Partnership

PURPOSE: Our preclinical medical curriculum features close collaborations between basic scientists and clinicians in the design and delivery of large group and small group learning. We employ this same team-based approach to create high fidelity simulation opportunities that are longitudinally integrated into the first two years of our curriculum. In addition to clinical skills development, our sessions reinforce the learning of foundational science concepts in a clinical setting.

METHODS: Teams of basic science and clinician educators collaboratively write cases, run the simulation exercises, and lead post-simulation debriefing sessions for our firstand second-year students $(n=90)$. Basic scientists serve as manikin operators, and clinicians act as embedded participants in the role of a nurse. Debriefing focuses primarily on reinforcing concepts related to pharmacology and pathophysiology of disease, and secondarily on teamwork and clinical assessment skills.

RESULTS: Both basic science and clinical faculty have successfully collaborated in the planning and execution of high fidelity simulations. From post-session evaluations, a high proportion of students agreed or strongly agreed with statements that the exercises increased confidence in communicating as part of a team $(89.0 \%)$, that the debriefing contributed to learning (84.6\%), and that the overall quality of the exercise was high (93.5\%). Faculty also agreed that simulations were positive and collaborative experiences $(86.7 \%)$, that debriefing in collaborative teams is more beneficial ( $85.7 \%$ ), and that simulations have helped in curriculum integration efforts (71.4\%).

CONCLUSION: Student and faculty perceptions of simulation exercises are overwhelmingly positive. Based on student and faculty feedback, team debriefing was found to be more effective in small groups, with a clinician and basic science educator working as co-facilitators. Our collaborative approach to simulation appears to have had a positive effect on student learning, and collaborations have resulted in better implementation and integration of simulation longitudinally in the curriculum.

SOME LESSONS LEARNED FROM THE PLANNING, ADMINISTRATION, AND IMPLEMENTATION OF A MEDICAL SCHOOL REFLECTIVE WRITING PILOT PROJECT

Kathy Pittman

Ohio University

PURPOSE: Literature supports the need for strong student self-refection skills (Friedman et al., 2001; Hargreaves, 2016; Konopasek, Norcini and Krupat, 2016). The Heritage College of Medicine is exploring how student selfreflection, self-direction, and critical thinking skills can be strengthened through written, thoughtful student reflections that will serve them well throughout their careers and with a better understanding of what the Core Competencies and Entrusted Professional Activities (EPAs) mean to them now, and as future doctors. This poster describes a pilot project to implement reflective writing into an integrated medical school curriculum.

METHODS: Goals of the 10-week project were to demonstrate student critical thinking, self-directed learning, and reflection skills; to observe student/coach relationship dynamics, and to introduce students to the 7 Core Competencies and EPAs. Nine clinician coaches were paired with nine second-year students and asked to meet at least 3 times, face-to-face or via email. Students completed a written 
reflection related to a clinical experience, scored by each clinician using a rubric based on the 7 Core Competencies and the Dreyfus Model. Clinician/student comments about the program were collected and analyzed qualitatively.

RESULTS: Engagement between students and clinicians occurred over the 10 -week period but needed nurturing. Clinicians used the scoring rubric but most found it to be detailed and difficult to follow. Students and clinician comments indicated increased appreciation for self-reflection and knowledge related to the Core Competencies and EPAs. A few students questioned the relevance of reflective writing. The need for a program coordinator was identified.

CONCLUSION: Reflective writing is not so much about "assessment," as it is about engagement in the process, building the skills of critical thinking, self-directed learning, and reflection. This project provided insights for designing a medical school reflective writing program important for future curricular design.

\section{REAL PATIENT ENCOUNTERS ENHANCE PRE- CLINICAL PATHOPHYSIOLOGY AND PHAR- MACOLOGY EDUCATION IN BASIC SCIENCE COURSES}

\section{Laurel Gorman I Feroza Daroowalla}

\section{UCF College of Medicine}

PURPOSE: To date, preclinical pathophysiology and pharmacology courses have embraced case-based learning (CBL) using paper or computer-based cases to facilitate transference and contextual learning. Medical learners may perceive the design of these exercises to be static or irrelevant, with some becoming disengaged. As medical schools are incorporating more patient centered pedagogy at all stages of curriculum, we have successfully used different models for patient encounters to support engaging foundational science education. The objective of this poster is share different models and report student perceptions of positive educational value. METHODS: We reviewed classroom sessions and materials that incorporate real patients during second year medical school pulmonary and endocrine-reproductive integrated systems modules. To evaluate perceived impact, we retrospectively reviewed the responses on the end-of-module evaluation and comments on patient encounter sessions (four survey items specifically targeted learning, clinical relevance, engagement, and interaction method).

RESULTS: Session designs included: 1) Physician interviewing patient in front of class with the class developing illness script in small groups; 2) Patients presenting their story and perspectives, with a faculty facilitator, in a student question forum; 3) Short real patient videos during didactic sessions. Most students ( $\mathrm{N}=116$ answered, 99\%) agreed that patient encounters enhanced learning and clinical relevance of pathophysiology and pharmacology (90\%) and module engagement (83\%). They agreed asking real patients questions was valuable to learning (82\%), although fewer agreed (64\%) about the learning values for patient videos. In open comments, positive themes emerging included empathy, humanization of basic sciences, role-modeling (the physician interview model), and real world relevance.

CONCLUSIONS: Students perceived high educational value to using real patient encounters to support pathophysiology or pharmacology preclinical teaching. All methods described in our poster may be implemented elsewhere, although some approaches may be better for developing specific traits like empathy or role-modeling positive physician-patient interactions.

\section{MEDICAL STUDENT ACADEMIC PERSISTENCE AND PERFORMANCE IN ONLINE LEARNING ENVIRONMENT DURING THE COVID-19 PAN- DEMIC LOCKDOWN}

Mohsin Syed I Noor Akhter I Mohamed Ibrahim I Laura C. Stanley

University of Arkansas For Medical Sciences

OBJECTIVES: The purpose of this study is to assess the academic adaptability and success of medical students and faculty who in emergency lockdown during the COVID-19 pandemic suddenly switched to a completely online learning format.

METHODS: One-way ANOVA was used to compare pandemic and pre-pandemic grades of medical students at the University of Arkansas for Medical Sciences in the brain and behavior Spring module. Students' test scores related to perception of persistence levels was studied using correlation analysis. Finally, a regression analysis was performed to examine prediction factors of medical student course grades during COVID-19.

RESULTS: Students' grades in the module during the COVID-19 were significantly higher compared to the grades in recent prior years at the $\mathrm{p}<.001$ level $[\mathrm{F}(3,692)=9.08]$. Pearson product-moment correlation results showed a strong and positive correlation between students' persistence level $(\mathrm{M}=3.46, \mathrm{SD}=.997, \mathrm{n}=79)$, and their module grade during COVID-19 $(\mathrm{M}=258.777, \mathrm{SD}=14.6878, \mathrm{n}=79), \mathrm{r}=.33$, $\mathrm{p}=<.01$. Multiple linear regression was able to account for $14 \%$ of the variance in students' module grades and was statistically significant at $\mathrm{p}<.05$.

CONCLUSION: We conclude that student's tenacity to adjust to a new learning environment together with module directors and faculty successfully employing remote education methods met learning challenges in the emergency 
of the pandemic where students maintained a high level of academic success.

\section{THE VALUE OF AN INTERPROFESSIONAL CLINI- CAL LABORATORY SCIENCE EXPERIENTIAL ACTIVTY IN A PASS-FAIL MEDICAL SCHOOL DURING THE COVID-19 PANDEMIC}

Daniel R. Webster | Tammy Carter | Koy Kubala | Jessica Brashear I Corey Swackhammer I Daniel Wood

Texas Tech University Health Sciences Center-Lubbock

PURPOSE: We hypothesized that, during this COVID-19 crisis, a small-group active-learning activity would provide benefits to student wellness while still providing a meaningful learning experience.

METHODS: A hypothetical clinical scenario was developed. MS1 volunteers were divided into two learning groups (Experiential or Self-Directed). Students in each group were presented with the same clinical case and then asked to develop a preliminary diagnosis and three laboratory tests to either affirm or rule out their diagnosis. The SelfDirected students were then given pertinent test results for their patient and unfettered access to any resources needed in order to refine their diagnosis. Experiential students spent time in a clinical lab where different lab tests were explained by Clinical Laboratory Sciences (CLS) students. The Exp students then participated in small-group discussions with aid from the CLS students. Both groups were required to submit a final diagnosis plus recommended lab tests at the end of the activity. All were quizzed periodically on the scenario in order to test retention of concepts developed during the exercise.

RESULTS: The Experiential and self-directed learning groups performed similarly on all of the quizzes. Compared to the SDL students, those in the Experiential group felt the instruction offered by the activity was more effective (94\% vs 59\%), that they were better prepared for the remainder of medical school ( $87 \%$ vs $59 \%$ ) and were more likely to strongly recommend this activity to future medical students (69\% vs $47 \%)$.

CONCLUSION: Learning approach did not affect student performance. However, enjoyment and satisfaction were greater in the Experiential group. In the midst of a global pandemic that is affecting all aspects of medical students' lives, safe and effective methods of maintaining experiential learning in the pre-clinical years may have great benefit in keeping students engaged and fulfilled.

MAKING CONNECTIONS EXPLICIT: THE EFFECT OF SELF-EXPLANATION AND WORKED
EXAMPLES ON COGNITIVE INTEGRATION OF BASIC AND CLINICAL SCIENCES

Kristina Lisk

Humber College Institute of Technology and Advanced Learning, Toronto, Canada; The Wilson Centre, Toronto, Canada; University of Toronto, Toronto, Canada

PURPOSE: A growing body of experimental evidence suggests that learners benefit from individual teaching sessions when the instructional materials are designed to support cognitive integration. However, there is limited research on specific strategies that educators can use to support learners in achieving cognitive integration. In this study we examined the effect of integrated basic science instruction combined with either self-explanation or worked examples on novices' learning diagnosis of musculoskeletal pathologies.

METHODS: Participants $(n=37)$ were randomly assigned to either the self-explanation (SE) or worked example (WEx) learning condition. All participants were explicitly taught the clinical features and underlying basic science mechanisms of four musculoskeletal pathologies. After each pathology was presented, participants read a short clinical vignette that depicted that specific disease. Participants in the SE group were then prompted to use their basic science knowledge to self-explain why the clinical presentation represented a person with this disease. In comparison, participants in the WEx group were asked to read an additional paragraph that highlighted the underlying anatomical pathology for each of the clinical features presented in the vignette. Immediately after learning and 1-week later, all participants completed a diagnostic accuracy test followed by a basic memory test. RESULTS: The results showed that the SE group scored significantly higher on the diagnostic accuracy test compared the WEx group $(p=0.037)$. No difference between groups was observed on the memory test $(\mathrm{p}=0.115)$.

CONCLUSION: The findings of this study demonstrate that holistic self-explanation is an effective learning strategy that can be used to support the development of novices' integrated knowledge of disease categories. It is suggested that even when learners have minimal clinical knowledge, self-explanation is a strategy that supports active processing and the development of a more coherent mental model in comparison to the use of worked examples. FUNDING: IAMSE Scholarship Grant

IMPARTING QUALITY IMPROVEMENT EDUCATION TO UNDERGRADUATE MEDICAL STUDENTS

B. Wahi-Singh | Z. Alexander | L. Chuk | F. Hunter | B. Moncur I Y.H. Tan I M. Teo 
University of Edinburgh

PURPOSE: Both the UK GMC and US AAMC have stressed the importance of medical student education in quality improvement (QI). While this is a required activity for junior doctors and graduate medical programs, it is less commonly seen in undergraduate medical education. Receiving staff and patient feedback on the patient journey is a valuable QI activity to actively teach medical students in a time limited project.

METHODS: At the University of Edinburgh, semester-long student-selected components (SSC) allow undergraduate medical students to engage in supervised, active, collaborative learning. As part of an SSC project, seven first year medical students undertook semi-structured interviews with cardiac critical care staff and patients to identify quality shortfalls in a mapped sample patient journey. Feedback was also obtained from the medical students on their educational experience.

RESULTS: Within a 10-week duration the students interviewed 63 respondents to identify areas of the patient journey requiring improvement. Areas identified included handover, delirium, and pain protocols. Further, more focused QI projects have been defined as a result of this work. In addition, responses on a feedback survey from the current medical students demonstrated their belief that they gained valuable insight into quality improvement. Most also expressed plans to engage in additional QI projects in the future.

CONCLUSIONS: It is possible to use time-limited active learning programs such as an SSC, which allows students to conduct faulty-supervised projects, to expose students to QI. Students report an improved understanding of QI process and desire to engage in additional QI work in the future with this educational activity. In the future, it would be worth objectively measuring the impact of such projects on student understanding of QI and whether an interest in QI is sustained.

\section{A NOVEL METHOD OF INTEGRATING FOUNDA- TIONAL AND CLINICAL SCIENCES IN THE CORE CLERKSHIPS}

\section{Bindu Menon I Tyrone Layson I Deepa Mukundan}

University of Toledo College of Medicine and Life Sciences

PURPOSE: Principles of cognitive science can facilitate integration and retention of basic science concepts during the clinical years. Studies have shown that integration improves diagnostic accuracy and understanding of key clinical features. The aim of this study was to determine if the re-introduction of core foundational science concepts strengthens student's learning during their clinical rotations.
METHOD: Our approach was based on facilitating student's construction of cognitive schemata to facilitate the development of clinical reasoning. A patient simulation session on diabetic ketoacidosis in the pediatric clerkship was chosen to pilot the program. The session starts with a small group of students working their way, using a patient simulator, through the physical examination and laboratory test results to identify the most probable differential diagnosis. Following this, the faculty experts facilitate student discussions forcing them to connect important physiological and pathophysiological concepts to the clinical presentation, diagnosis, and management of the disorder.

RESULTS: The students described the new approach as a great positive step with $89 \%$ strongly agreeing, (11\% agrees and no one disagrees) that it was helpful. Preliminary studies using the NBME item analysis report of the subject exams showed an improvement in the student performance following the introduction of this didactic session. The strength of the approach was that students could solidify their knowledge by aligning diagnosis and management with the pathophysiology in the setting of a team teaching facilitated by clinical and basic science faculty. The limitation was that study was conducted in one clerkship.

CONCLUSION: This approach can be easily introduced provided the basic science faculty are available to work in the clinical setting along with clinical faculty, in the absence of which a video presentation or online module is a viable alternative. This is an innovative and practical approach to introduce basic science content in the clinical years.

\section{MEDICAL SKILLS DURING THE PANDEMIC: EXPERIENCE OF PRE-CLINICAL MEDICAL STUDENTS}

Damaris Rosado, PA-C I Gordon Woods, MD । Brad Fuhrman, MD I Laura Gorby, MSN, RN I Dale Quest, PhD I Thwe Htay, MD I Houriya Ayoubieh, MD

Texas Tech University Health Sciences Center at El Paso, Paul L Foster School of Medicine

PURPOSE: The COVID-19 pandemic changed the way medical education is delivered worldwide. The Medical Skills Course in the preclinical curriculum at the Paul L. Foster School of Medicine was converted to a hybrid learning model. Physical examination skills were taught with social distancing measures using an instructor who was physically present or through videoconferencing. Standardized patient encounters were changed to virtual experiences. The purpose of this study was to evaluate the preclinical students' perceptions of their experience learning medical skills through hybrid instructional methods. 
METHODS: We developed and administered an anonymous survey that included ratings of criteria using modified Likert scales and narrative responses to open-ended questions. Course evaluation data for learning activities in the Medical Skills Course delivered during the 2020-2021 academic year were also reviewed.

RESULTS: Sixty-four of 115 (56\%) first year medical students (MS1s) and 58 of 108 (54\%) second year medical students (MS2s) responded to the survey. Six of 64 (9\%) MS1s and 17 of $58(29 \%)$ MS2s agreed that learning physical examination skills through videoconferencing was adequate for their learning $(\mathrm{p}<0.0019)$. Forty-one of $64(64 \%) \mathrm{MS} 1 \mathrm{~s}$ and 49 of $58(84 \%)$ MS2s agreed that virtual SP encounters were adequate for their learning $(\mathrm{p}<0.0152)$. Thematic analysis of the open-ended questions demonstrated a preference for in-person components. The student's overall satisfaction with the course was $95.5 \%$ for MS1s and $96 \%$ for MS2s compared to $99 \%$ and $98.7 \%$ for historic cohorts.

CONCLUSION: A hybrid model for Medical Skills was feasible and did not significantly change the overall student satisfaction with the course. Student perceptions of virtual SP encounters were more favorable than learning physical examination skills through videoconferencing. MS1s are less likely than MS2s to find virtual aspects of the course adequate for their learning, and we recommend that educators take this into consideration when designing hybrid curricula.

\section{MULTIFACETED ACADEMIC TOOL: A PROTOCOL FOR TEACHING \& LEARNING}

\section{Maureen Hall | Daria Ellis | Priya Dattathreya}

\section{Ross University School of Medicine}

PURPOSE: A multifaceted academic tool was designed to support faculty facilitated teachings and students' learning through reflective and guided protocols. Using a similar approach of the learning cycle, we established seven steps as academic tools for both faculty and students. These seven steps are referred to as the 7Ps for ease of retrieval which include -Plan, Preview, Participate, Process, Practice, Performance and Pause.

METHODS: Using a qualitative approach, a focus groups was conducted with faculty to discuss direct experience with students reflecting on overall academic outcomes. In addition, students took an inventory survey tool of items regarding an approach on how learning took place within the curriculum.

RESULTS: The results of comparative analysis of survey results revealed that students' use of the guided protocol allowed students to organize learning of large volume of contents within the medical institution and thus had more favorable academic outcomes than those students who did not. Faculty were more confident teaching metacognitive strategies to students. Students established a sequence of daily study routine that aided successful self-regulated learning in a fast-paced academic environment.

CONCLUSION: The multifaceted academic tool provided benefits to teaching and learning for both faculty and students of the curriculum. It not only takes the students' reflection and teaching faculty guidance to support student's learning but also, the institution's curriculum design.

\section{LEVERAGING LEARNING SCIENCE AND PLATFORM-BASED DELIVERY: A PANDEMIC- PROOF SOLUTION TO OPTIMIZING MEDICAL EDUCATION}

\section{Peter Horneffer}

\section{All American Institute of Medical Sciences}

PURPOSE: Platform-based administration and tracking of didactic content offers distinct advantages with respect to implementing evidence-based strategies from Learning Science. The COVID-19 pandemic demonstrated another distinct advantage of platform-based administration of curricular materials- resiliency in the face of unavoidable disruptions to normal operations. This presentation will describe how a small medical school in an underdeveloped country used its platform-based curricular approach to continue operations during that's country's pandemic induced lockdown.

METHODS: Students and faculty in this under-resourced setting had converted from a paper-based traditional teaching model to a platform-based curricular delivery system 6 months before the onset of the COVID-19 pandemic and its resultant national shelter-at-home order. As the faculty and students had already adopted the platform in order to benefit from the application of retrieval-based learning strategies, classes simply had to migrate to a synchronous video format for "in-class" activities.

RESULTS: While the sudden conversion to online synchronous classes was not without its challenges, both the faculty and the students found certain advantages to the new approach. Furthermore, faculty who had been hesitant supporters of the effort to use a platform-based approach prior to the pandemic became advocates for the approach as the numerous advantages for both learning and teaching became increasingly apparent including the assured continuity of instruction. The resulting comfort in using technology has afforded an even more progressive approach to curricular delivery with the adoption of a "flipped-classroom" approach and a focus on interactive learning formats.

CONCLUSION: Platform-based curricular delivery not only provided for a more effective educational process but 
proved remarkably resilient in the face of a widely disruptive pandemic. Given the likelihood for further disruptions from a variety of natural causes, serious consideration should be given for all medical schools to adopt a platform-based approach to delivering their curricula.

\section{Other}

\section{MULTIPLE MINI-INTERVIEW MAPPING: A NEW METHOD TO PERSONALIZE THE MMI PROCESS FOR MEDICAL SCHOOLS}

\author{
Nicholas A. Patete I Kyle Bauckman Ph.D. \\ Dr. Kiran C. Patel College of Allopathic Medicine
}

PURPOSE: The process of applying to medical school is extremely competitive with only $41 \%$ of applicants matriculating into an American allopathic medical school. Despite a large application pool, medical schools have the difficult task of selecting future physicians who match the individual school's mission and objectives. A holistic review approach is important to select not only applicants who will make good medical students, but applicants who match the individual medical school's goals and mission statement.

METHODS: We propose a new Multiple Mini-interview (MMI) strategy to better personalize the process. The malleability of the MMI is beneficial as it allows schools to provide a holistic review which is not "one size fits all", but instead is personalized to individual school goals and mission statements. By mapping MMI questions to school Medical Education Program Objectives (MEPOs), we rate applicant's MMI performance based on the individual's performance in each identified MEPO. Performance in each MEPO is compared to other applicants' performance and to the average matriculated student's performance. This method is intended to improve the confidence in scoring and reviewing applicants.

RESULTS: MMI questions utilized by the admission committee were reviewed and matched with MEPOs that accurately represent the MMI question. Interviewer score sheets were updated to include assessment components for the selected MEPOs. Data was processed and analyzed via radar chart for admissions committee review. Admissions committee members were surveyed on the usability of MEPOs in assessing MMI results.

CONCLUSIONS: Mapping the MMI process akin to mapping medical school curriculum provides a more nuanced and informed decision. The accessibility of the data in radar chart format helps to identify individual attributes of the applicants that would otherwise be glossed over. Future investigations will focus on tracking the benefit of this MMI process toward providing well-rounded and diverse matriculants.

\section{DOES BURNOUT AFFECT STUDENTS' ABILITY TO RECEIVE FEEDBACK?}

\author{
Carrie Yuan
}

Idaho State University Department of Physician Assistant Studies

PURPOSE: Feedback is one of the most powerful influences in learning and achievement; however, the perception of how the feedback is given can lead to either positive or negative consequences for the feedback receiver. Previous studies have demonstrated how to best utilize feedback such that it becomes a powerful tool in education. Burnout is a state of feeling physical, emotional, and mental stress often developed in response to chronically stressful conditions. This study aims to investigate if there is a relationship between students' feedback perception and correlation with burnout. METHODS: To measure burnout, the abbreviated Maslach Burnout Inventory (aMBI) was utilized. The aMBI consists of nine questions assessing the following categories: Emotional Exhaustion, Depersonalization, and Personal Accomplishment. Feedback perception is assessed via survey carefully designed based on previous literature to assess the following categories: judgement, confidence, defensiveness, vulnerability, emotional response, and perceived power of the feedback giver. Survey was distributed to didactic year Physician Assistant students. Regression models were constructed to investigate the relationship between feedback and burnout. Student's t-test was used determine statistical differences. Survey validity analyzed using SPSS.

RESULTS: 94 students across 4 institutions participated in the study. Results demonstrated correlation between feedback reception and degrees of burnout. Using feedback scores, students were designated as either "excellent, good, moderate, or poor" feedback receivers. Burnout components were further analyzed between feedback receivers. New to this study is the consideration of the impact of the pandemic on feedback reception and burnout.

CONCLUSION: Feedback is an important aspect of education. Factors to consider in improving student feedback reception includes strategies on behalf of educators and of students. Our current study suggests that students are not receiving feedback consistently or frequently. The quality of the feedback plays an important role. This study identified areas of improvement for both students and educators and proposes potential changes in feedback delivery. 
MICROMANAGEMENT DURING CLINICAL SUPERVISION: A HIDDEN CURRICULUM AFFECTING AUTONOMY AND COMPETENCE OF TRAINEES

Bhawana Arora, MD I Rakesh Surapaneni, MD I J.M. Monica van de Ridder, $\mathrm{PhD}, \mathrm{MSc}$ I Anuradha Lele Mookerjee, MD I Vijay Rajput, MD

College of Human Medicine, Michigan State University, Grand Rapids, MI I Internal Medicine Residency- Round Rock Program, Texas A\&M College of Medicine I Cooper Medical School of Rowan University, Camden, NJ I Nova Southeastern University, Dr. Kiran C Patel College of Allopathic Medicine, Ft. Lauderdale, FL

PURPOSE: Entrustable Professional activities are the roadmaps for growth and development of trainees in Graduate Medical Education. All trainees require adequate supervision while being provided appropriate autonomy and entrustment. Micromanagement is defined as a supervisory style of "hovering" and directly commanding all the details, rather than giving space to the trainee assigned to perform the task. The literature on micromanagement is sparse in medical education. The goal of our research is to identify characteristics of micromanaging behavior, its causes, and consequences on the learning environment.

METHODS: A search in PubMed " (micromanage*[Title/ Abstract]) and ((undergraduate medical education [MeSH Major Topic]) or (Graduate Medical Education [MeSH Major Topic]))" revealed only 6 results. With help of a librarian, we explored the literature in medical education, business, nursing, sociology, and psychology. After identifying key-articles, we used the 'snowball method' to identify more relevant literature. The LinkedIn Learning, MedEdPortal and MedEdPublish databases were used to identify materials on this topic.

RESULTS: Micromanaging behaviors consist of a combination of traits including asking for frequent updates, scrutinizing details, and taking pride in correcting others' mistakes. The causes of such behavior could be secondary to organizational structure, faculty insecurities, distrust, and fear of failure. The consequences could lead to poor supervisorlearner relationship, lower team morale and overall threaten trainee's wellbeing. We have developed a conceptual framework of micromanagement in clinical settings that describes a 'static zone of safety', that cannot be adjusted to the trainees' needs. The adaptive attendings on the other hand create a 'flexible zone of safety', which they can adjust based on their trust in the trainees' competence.

CONCLUSIONS: Micromanagers can be detrimental to learners' autonomy, and their overall competence. They often impact the team in a negative manner. There is a need for strategies for recognition, mitigation of micromanagement at trainee, faculty and institutional level.

\section{MEDICAL STUDENTS AS HEALTH- CARE EDUCA- TORS IN AN ONLINE COMMUNITY ENGAGEMENT PROGRAM DURING THE COVID-19 PANDEMIC}

Valeria Urbina | Jorge Cervantes I Melanie Olivas Longhurst I Niti Manglik | Curt Pfarr I Jessica Chacon I Houriya Ayoubieh

Texas Tech University Health Sciences Center El Paso, Paul L. Foster School of Medicine

PURPOSE: A group of medical students and faculty at Paul L. Foster School of Medicine, Texas Tech University Health Sciences Center at El Paso, formed the El Paso Health Education and Awareness Team (EP-HEAT). Under faculty guidance, students assumed the role of health-care educators, with the goal of providing the evidence-based and updated information about COVID-19 to the local community and beyond. We surveyed students about their perceptions regarding their experience participating in the EP-HEAT.

METHODS: Students researched topics concerning the COVID-19 pandemic, summarized the information in easy to-read downloadable pamphlets and hosted live virtual health fairs. Students led the discussions, encouraged the audience to interact, and responded to questions. The role of faculty moderators was to oversee the development of the educational material and to provide feedback and support. To assess the students' experience in this project, a Likert-based survey was used to measure the benefits from participating in EP-HEAT. Additional open-ended questions allowed students to summarize their experiences in narrative form.

RESULTS: Fourteen of nineteen EP-HEAT students (73.6\%) completed the survey. Most of the students agreed that having the opportunity to teach during medical school with direct observation from faculty is useful for their careers. Prior to EP-HEAT participation, $40 \%$ of students felt prepared to answer questions regarding SARS-COV2/ COVID19, compared to $92 \%$ of students after participation. Majority of students reported that the virtual health education fairs enhanced their confidence in explaining healthrelated information $(87.9 \%)$, communication $(92.9 \%)$, and teaching skills $(85.8 \%)$.

CONCLUSION: Student participation in EP-HEAT enhanced their confidence in explaining health-related information to the public. Our results should encourage more in-depth exploration of teaching opportunities for students as part of their medical 
school curricula, and community engagement projects as a potential avenue for student-teaching implementation.

\section{YOU HAVE TO CHANGE TO STAY THE SAME}

Robert Hage I Matthew Carvey

\section{St. George's University}

PURPOSE: The above adage is credited to Willem de Kooning, a Dutch-American artist. A similar statement "If we want things to stay as they are, things will have to change", was made by the character Tancredi in the novel Il Gattopardo. Adjustment is what medical schools have done to sustain business during the COVID-19 pandemic. Ensuring continuity in medical students' education in a time of social distancing guided instruction towards communication through electronic media, simplified by pre-existing online infrastructures and software. The human touch in medicine was substituted with telemedicine, the i-Human platforms, and additional innovative solutions all meant to be temporary measures until the pandemic allows medical schools to return to prior traditional teachings. Medical schools "forced" amendment to customary education has permitted both business and teaching innovations, enhancing curriculum development and its medical students for a future in post-pandemic conditions.

METHODS: An analysis of institutional activities before and throughout the COVID-19 pandemic is presented, indicating the changes which occurred. Supplementary to this analysis is a directional prediction schools of medicine may inherit once students return to campus.

RESULTS: Administration wise, the opportunistic benefits are focused on increasing student numbers and maximizing resources, whereas the teaching aspect is attentive to quality control via ZOOM sessions, improved student advising, multiple methods of delivering small group interactions, and expanding office hours with students.

CONCLUSIONS: Institutional and teaching alterations initiated by COVID-19 will doubtfully return to their pre-pandemic state. The learning curve has been comparably steep for faculty and students; resistance to change has ceased, and a new standard is established. We have changed to remain the same when considering business traits; however, will the medical schools' transformation towards a new means of education lead to success or disappointment in student achievement?

DEPRESSION, ANXIETY AND STRESS DURING THE INITIAL 6-MONTH TRANSITION PERIOD ON TWO REGIONAL MEDICAL SCHOOL CAMPUSES
Jeffery Fritz I Craig J. Hanke

Medical College of Wisconsin

PURPOSE: Depression, anxiety and stress levels were measured at two regional campuses during the initial 6-month transition to medical school. The curriculum at these two campuses was nearly identical during this time, however student reflective practices were offered at one campus. We describe the findings of learner self-reported levels for depression, anxiety and stress at both campuses and learner perceptions of longitudinal reflective practices during this transition.

METHODS: Students at the Medical College of Wisconsin Regional Campuses were surveyed using the DASS2 1 instrument as a quantitative measure to assess levels of depression, anxiety and stress during the first 6-months of medical school. DASS21 surveys were distributed, collected and scored in August, November and February at the start of learning modules. One campus utilized longitudinal reflective practices with four narrative assignments during this time frame. Student satisfaction with the reflection exercises was surveyed afterward. This study has institutional IRB approval.

RESULTS: DASS21 surveys were completed by $95 \%$ of the student cohort at both campuses. Average depression, anxiety and stress scores increased in November and February compared to the initial August survey with minor differences between the campuses. November showed the greatest increase in depression, anxiety and stress scores with the stress category showing the greatest relative increase. Students participating in longitudinal reflective exercises noted that the practice eased their transition to medical school, and they felt cared for by their faculty. These students also noted reflective writing did not play a role in mitigating their feelings of depression, anxiety or stress.

CONCLUSIONS: Depression, anxiety and stress levels increased similarly during the first 6 months of medical school at two regional campuses. Longitudinal reflective writing practices did not mitigate these increases but appear to have a positive effect on student transition to medical school and learner-educator interaction.

FACILITATORS AND BARRIERS TO COLLABORATION IN A PROGRAM STRUCTURED TO BUILD TOOLS TO ENHANCE STUDENT COGNITIVE INTEGRATION

Tracy B. Fulton I James L. Nixon I Amy L. Wilson-Delfosse | David M. Harris | M. Robin English | Khiet D. Ngo | Leslie H. Fall 
University of California, San Francisco School of Medicine, San Francisco, CA 94143 I University of Minnesota Medical School, Minneapolis, MN 55455 | Case Western Reserve University School of Medicine, Cleveland, OH 44026 I University of Central Florida College of Medicine, Orlando, FL, 32708 I Louisiana State University School of Medicine, New Orleans, LA 70112 | Loma Linda University School of Medicine, Loma Linda, CA 92354 I Aquifer and Geisel School of Medicine at Dartmouth, Hanover, NH, 03755

PURPOSE: We recruited teams consisting of basic scientist educators, clinical educators, and students from US schools to contribute to the development of a library of tools (Virtual Patient Cases and Integrated Illness Scripts) designed to support medical students' cognitive integration of basic science concepts during study of clinical medicine. We conducted a program evaluation to examine the participants' characterization of their experience. Research questions focused on facilitating factors and barriers to collaboration within teams, and the impact of the experience on participants, using a conceptual framework situated in sociocultural learning theory and communities of practice.

METHODS: Twelve medical schools participated. Tool development occurred in cycles, using a design thinking and rapid prototyping approach, and templates previously developed. Basic scientist and clinical educator leads (24 total) and additional faculty participants (22) responded anonymously to Qualtrics surveys. We took a constructivist approach to assessing the survey responses, conducting thematic analysis to identify codes and themes related to our research questions and framework.

RESULTS: Initial theme identification suggests facilitators of integrative team collaboration include logistical factors, effective local leadership and engagement, individuals working outside their comfort zones, role checking, and clear expectations both internally and externally. Barriers included time, unclear/insufficient instructions, poor engagement from team members, and disagreement on level of detail. Participants identified that the work they did with their team had positive effects on their knowledge organization, their professional growth, and that this experience would have a major impact on their future teaching.

CONCLUSIONS: Teams described practices that supported or barred their navigation of traditional boundaries between disciplines and specialties, including use of students as brokers. We suggest future scholarship be directed at faculty development focused on these practices to support cognitively integrated curricular design.

\section{LAUNCHING A TEAM SCIENCE TRAINING INITIA- TIVE: INITIAL STEPS IN CREATING THE FACULTY DEVELOPMENT PLAN}

Carol Elam I Michelle J. Jones I Thomas Kelly I Susan Smyth | Erin Haynes | Hilary Surratt | Robert Kegebein | Victoria King

University of Kentucky

PURPOSE: To confront increasingly complex areas of inquiry, biomedical research is transitioning to a team science model that brings together interdisciplinary experts in integrated research teams to pursue scientific discovery. How can clinical and translational science workforce development and training programs best assist investigators in transitioning from roles as solo researchers to members of high functioning teams?

METHODS: Institutional investigators supported by a National Center for Advancing Translational Research (NCATS) Clinical and Translational Science Award sought to enhance the preparation and productivity of evolving clinical and transitional research (CTR) teams. A planning committee comprised of a research administrator and translational research leader, two department chairs, a management and interpersonal skills trainer, an educator, a qualitative researcher, and an evaluator was created. Committee members accepted the charge of offering a team science training program; weekly virtual committee meetings to facilitate planning were scheduled. Following a literature review and identification of model training programs, the committee drafted preliminary goals for the project and created a needs assessment instrument to determine topics of interest and concern to CTR investigators and trainees.

RESULTS: Planning committee members identified critical team science resources made available by NCATS, the National Institutes of Health and the National Cancer Institute. Institutional approaches to team science training were determined through the literature review. Essential team science competencies defined by the University of Pittsburgh Clinical and Translational Science Institute were adapted for development of learning objectives for our local training initiative. The initial target audience selected for a pilot of a team science training component was early career investigators, with training outcomes specified.

CONCLUSIONS: We are offering our initial pilot team science training in February 2021. We will share pilot findings, and a roadmap for our comprehensive training program for all CTR researchers and trainees, projected for full implementation by 2022 .

A PILOT EDUCATOR CURRICULUM USING TED MASTERCLASS TO DEVELOP SKILLS IN PRESENTING MICRO-LECTURES: LESSONS LEARNED 
Holly West, DHEd, PA-C I Kathleen Everling, PhD

University of Texas Medical Branch

PURPOSE: In this digital age, students desire to receive information in short bursts which focuses their attention without the cognitive overload of the longer, traditional lecture. Health professions educators need training on how to create and deliver these micro-lectures highly focused presentations on a well-defined topic. Using TED Masterclass as a skills training platform, we built a virtual 6-month educator development curriculum to enhance micro-lecture presentation skills. The purpose of this pilot was to evaluate program metrics, overall participant perceptions, and develop lessons learned to plan future cohorts.

METHODS: Twenty-three interprofessional educators began the 6-month program in May 2020 despite the COVID-19 pandemic. The program consisted of seven self-paced asynchronous TED Masterclass skill-building lessons along with three, hour long virtual Accountability Sessions facilitated by the authors. These sessions focused on learned skills and application to health professions education. At the culmination of the program, participants delivered a TED-style micro-lecture for feedback.

RESULTS: At the end of the 6-months, 10 (45.5\%) educators completed the program and another $3(17.6 \%)$ completed at 7 months. Although $4(20 \%)$ withdrew, there are several on track to complete the program by the end of 2020 . Those who attended all three of the Accountability Sessions were more likely to complete the program on time. Positive feedback has been received regarding the Masterclass lessons, Accountability Sessions, and complete program.

CONCLUSION: Several lessons were learned in this pilot cohort which will be useful for planning the future Cohort 2, starting Spring 2021. In addition to continuing the group Accountability Sessions, Cohort 2 will have an assigned Accountability Partner and a mentor from Cohort 1. In an effort for more frequent presentation feedback, we will have participants present a "lightening-style" micro-lecture at the mid-point period. This feedback can be used to enhance their final micro-lecture presentation.

\section{THE EFFECT OF COVID-19 ON MEDICAL STU- DENTS' PURSUIT OF ADVANCED DEGREES AND PATIENT ADVOCACY}

Saloni Sachar, MS, OMS-II I Danielle C. Thor, MAUB, OMS-II I Tipsuda Bahri, MD

Touro College of Osteopathic Medicine-New York

PURPOSE: Although the COVID-19 pandemic has impacted healthcare workers tremendously, its effect on medical students is unclear. Medical students are training in a time when the profession plays a pivotal role. It is imperative to assess the pandemic's impact on students' professional goals, specifically in obtaining additional degrees and patient advocacy.

METHODS: Students participated in an anonymous online survey (Qualtrics) from September-December 2020 that was pre-validated and approved by Touro College Health Sciences IRB before administration. This survey contained 24 items with single to multiple answer choices ( 3 scales: specialty, practice, and priority) and corresponding comment section, 4 items with 5-point Likert-type scale (not important to extremely important), and 8 demographic items. A subset of questions pertaining to students' interest in patient advocacy, leadership, and obtaining advanced degrees were analyzed.

RESULTS: The response rate was 28\% (208/810; $38 \%$ males and $62 \%$ females). $3 \%$ of respondents considered obtaining additional degrees post-medical school, including MPH, MBA, and MHA. In addition, pre and post-COVID-19 pandemic mean Likert scales showed students perceived higher importance of physician advocacy (mean Likert scale 3.9 and 4.5 , respectively; $p$-value $\left.=2 \times 10^{\wedge}-10\right)$, public health and preventative medicine (mean Likert scale 4.2 and 4.6, respectively; p-value $=4 \times 10^{\wedge}-7$ ), and physicians to serve as community leaders (mean Likert scale 4.2 and 4.6, respectively; -value $=1 \times 10^{\wedge}-7$ ).

CONCLUSIONS: We report an increase in medical students' desire to pursue additional education outside of the traditional DO degree and a greater importance of physician advocacy and leadership. Student physicians training during a pandemic have witnessed hardships physicians have t0 face. As aspiring physicians in the current environment, medical students need to have a strong understanding of public health, presence in the community, and active part in legislative advocacy and development of public health policies.

\section{HUMANIZING THE BASIC SCIENCES: EXPLORING THE IMPACT ON EMPATHY AND LEARNING}

Irene Lee

\section{Duke-NUS Medical School}

PURPOSE: Bringing clinical relevance into basic science content helps to create explicit connection between basic sciences and clinical sciences. While varied meaningful efforts in the integration have been made, evidence suggests that students' retention of basic science knowledge and protecting empathic disposition are unsatisfactory. We propose a critical intermediary step: the "humanizing" of the patient experience to link basic science content and clinical relevance. Providing patient stories related to underlying basic 
science content supports narrative learning while the trigger of emotional arousal provides powerful connections between major concepts and contextual details, facilitating effective learning. The aim of this study was to explore the impact of providing patient stories related to the basic science content on learning and empathy.

METHODS: We conducted a pretest-posttest study with 63 preclinical students, randomly assigned into either unexposed or patient stories-exposed group. Patient storiesexposed group completed questionnaire containing links to patient stories, self-reflection notes and questions related to their perceptions in accessing the stories. Participants completed empathy survey at the beginning and end of the first semester. Mixed effect analysis were used to compare the empathy means score pretest-posttest in the two groups while the reflection notes were analyzed thematically. Learning gain was determined by comparing the difference in mean scores from the relevant course assessments in the two groups while controlling for previous academic performance.

RESULTS: Participants in exposed group showed increased empathy mean scores but without significant difference between the two groups. Difference in the mean learning scores between the groups were also insignificant. Aligning with the themes identified in the reflection notes, participants in exposed group agreed that patient stories helped them empathize with patients and see the clinical relevance of the associated basic science content.

CONCLUSION: While it was not conclusive that patient stories effectively promote learning and empathy, the reflection write-up indicated strong empathetic element.

\section{THE AFFECTIVE AND COGNITIVE EMPATHY SCORES OF FACULTY TEACHING AT AN OSTEO- PATHIC MEDICAL SCHOOL}

\author{
Bruce W. Newton I Michelle deBlaquiere I Marissa Krugh | \\ Godwin Dogbey
}

Campbell Univ. School of Osteopathic Med.

PURPOSE: To determine the degree of cognitive and affective empathy clinical and basic science faculty possess who teach undergraduate medical students at mid-eastern United States osteopathic medical school.

METHODS: Our faculty voluntarily took the Balanced Emotional Empathy Scale (BEES) and the Jefferson Scale of Empathy (JSE) surveys ( $n=98 / 375 ; 26.1 \%$ return). Since empathy levels are sexually dimorphic, sex was an important variable. Data were collected on degree type (DO, $\mathrm{MD}$ or $\mathrm{PhD})$, and further divided into clinical $(\mathrm{n}=76)$ and basic science faculty $(n=10)$, with 12 missing data. Clinicians indicted which specialty they taught/practiced. The specialties were broken into "Core" and "Non-Core" groups, with Family Medicine, Internal Medicine, Ob/Gyn, Pediatrics and Psychiatry representing Core specialties. The other 18 specialties were Non-Core, e.g., Radiology, Emergency Medicine. Scores were analyzed using SPSS.

RESULTS: There were no statistical differences in the JSE $(\mathrm{p}=.526)$ or BEES $(\mathrm{p}=.472)$ scores among the three groups. For DO's and MD's, Core specialty JSE and BEES scores were significantly higher (both $\mathrm{p}=.001$ ) than those practicing in Non-Core specialties. Women clinicians had larger JSE and BEES scores than men. Further analyses will compare faculty empathy scores to our medical student scores. Our faculty data will be compared to JSE data from faculty in several different schools and specialties.

CONCLUSION: Regardless of a faculty member's degree, cognitive and affective empathy scores do not differ among our medical school faculty. Once our data are compared to faculty at other schools, the "mind, body, spirit" osteopathic approach to medical care may show our faculty have higher empathy scores than faculty at allopathic schools.

\section{THE GROSS ANATOMY LABORATORY AT A CROSS-ROADS: ETHICAL, LEGAL, AND EDUCA- TIONAL IMPLICATIONS OF DIRECTING A HUMAN DISSECTION LABORATORY}

Richard A. Gonzalez I Lane P. Fortney | Sarah Garner I Alberto E. Musto I Matthew S. Myers I Breezy Wasko

Eastern Virginia Medical School I Eastern Virginia Medical School and Pathologists' Assistant Program, Tulane University School of Medicine I Virginia State Anatomical Program, Virginia Department of Health

PURPOSE: The process of human dissection has a rich history of ethical and legal paradigm shifts in western medicine. Despite technological and pedagogical innovations, dissection is still considered by many as the gold standard for teaching and learning anatomy. The dissection experience can be emotionally, psychologically, and physically demanding. It is through this formative experience that students and junior faculty learn powerful professional and ethical lessons. These lessons are taught not only by the process of dissection, but by the culture and environment created by the faculty and staff who partake in the anatomy experience. METHOD: A literature review was utilized to identify areas in which ethical, legal, and educational content intersects with management of the laboratory and anatomy experience. Comparisons of state anatomical boards and medical school curricula facilitate this contrast, and case examples from the literature provide a means for highlighting areas of discussion and recommendations of best practices for facilitating an ethical and professional experience. 
RESULTS: The anatomy experience can have positive and negative consequences on student and faculty professional development. Most influential are transparency and adherence to ethical and legal standards, the professional behavior of faculty and staff, and the conditions and support of the laboratory environment.

CONCLUSIONS: The culture and environment within the anatomy laboratory paves the way for the professional development of students and faculty during their formative years. Their personal experiences, their observations of professional interactions, and the treatment and handling of donors shapes how future clinicians will view and interact with their patients. In this respect, the anatomy laboratory must operate in an environment in which adherence to ethical and legal standards is of utmost priority to faculty, staff, and students. There must be a culture of accountability, protocol transparency, respect, and collaboration within a scholarly framework.

\section{IMPACT OF ECFMG'S 2023 DIRECTIVE ON CAR- IBBEAN MEDICAL SCHOOLS' SELF-EVALUATION AND CQI PROCESS}

Sateesh Babu Arja I Sireesha Bala Arja I Samir Fatteh I Krishna Teja Challa I Manoj Kumar Reddy Somagutta

\section{Avalon University School of Medicine}

PURPOSE: The numbers of Caribbean medical schools are increasing day by day, and the little is known about the Caribbean medical schools. The accreditation and regulation requirements vary from one island to another island, and in some islands, it is voluntary. Because of this, the announcement made by the Educational Commission for Foreign Medical Graduates (ECFMG) is significant. Starting in 2023, individuals applying for ECFMG Certification must be a student or graduate of a medical school that is accredited by an accrediting agency that is officially recognized by the World Federation for Medical Education (WFME). This study aims to find out the impact of accreditation on Caribbean medical schools' self-evaluation and to examine the continuous quality improvement elements across the Caribbean medical schools.

METHODS: This is a qualitative study, and data analysis was done by framework analysis. We employed the semistructured interview method to interview the academic leaders and faculty members from three different types of Caribbean medical schools; accredited medical schools, non-accredited medical schools, and a school never applied for accreditation. A total of six Caribbean medical schools participated in the interview process.

RESULTS: A total of 12 participants participated in the interview process. Results have shown that Caribbean medical schools are changing their educational and self-evaluation processes. In spite of Caribbean medical schools have a strategic plan and engaged in the change process, it lacks the continuous quality improvement. Self-study committees are constituted only at the time of accreditation site visits. CONCLUSIONS: Further studies are required to be conducted to investigate if the Caribbean medical schools are making changes as reactive changes to the accreditation site reports and to meet the accreditation standards, or are they trying to set up the culture of continuous quality improvement?

\section{THE "WHY" \& "WHAT FOR" OF TEACHING ACAD- EMIES FOR LEARNER-CENTERED EXCELLENCE}

\section{Shari Whicker, EdD | Alisa Nagler, EdD}

Carilion Clinic/Virginia Tech Carilion School of Medicine I The American College of Surgeons

BACKGROUND: The future success of academic medicine relies heavily on recruitment, development, and retention of faculty. However, faculty often don't feel supported in their teaching roles. While clinical contexts vary, health professions faculty share a common need to educate future professionals. Participation in a health professions academy allows providers to engage in professional development activities and build a community of practice. Medical educator communities have grown, yet no consistent model or mission is available for those looking to develop their own. This may be attributed to the variance in goals and missions. Authors sought to identify academy missions across the country to identify common themes and determine how academies can be leveraged to enhance professional development.

METHODS: At the authors independent organizations, they have developed unique teaching academies focused on learner-centered teaching excellence via the development of faculty skills as teachers, learners, and education researchers. At VTCSOM, an inclusive, interprofessional model was developed which differs from the honorific model of the ACS. In order to compare academies across the nation, authors disseminated a survey focused on the components of academy mission statements. Data was analyzed in aggregate to identify common themes.

RESULTS: Nineteen responses from academies across the US were received. "Professional development" and "development activities" were common themes within mission statements. Salient points included -research, faculty development, and development of a community of practice.

DISCUSSION: Data collected from the survey has been used to inform similarities and differences of professional development missions of academies. Identifying an 
intentional mission for individual academies is critical to success but sharing these unique goals at a national level allows for collaboration and the fostering of further growth. Teaching academy models can be easily be adapted as an approach to bringing faculty from different disciplines to develop and support each other in their roles as teachers.

\section{DEVELOPMENT OF WELL-BEING AND SELF- REGULATION COMPETENCE THROUGH EDUCA- TIONAL IMMERSION ACTIVITIES}

Miriam Turrubiates I Mary Ana Cordero I Silvia Olivares

Tecnologico de Monterrey

PURPOSE: The Well-being and self-regulation competence aim for the student to systematically implement pertinent physical, emotional, mental and economic self-care strategies, which allow them to develop in an environment of change and uncertainty, according to their personal and professional well- being project. At Tecnológico de Monterrey, Semana Tec is proposed, which is a curricular space of total immersion in activities that has the objective of contributing to the development of these competences. The aim of this study is to identify the development of the Well-being and self-regulation competence based on the Theory of Confirmation of Expectations of the activities "Ikigai: Build your dreams", "Building body and mind", "Press play to your well-being" and "I choose to be healthy".

METHODS: A quantitative, descriptive, and transactional method was applied. A questionnaire was developed and implemented as a pre-test and post-test. This instrument was validated by a focus group and the Cronbach's alpha resulted in 0.97. Fifty-five students answered the survey. For statistical analysis, the t-student test was used.

RESULTS: The activities "Ikigai: Build your dreams", "Building body and mind", "Press play to your well-being" and "I choose to be healthy" had a significant statistical difference on Well-being and self-regulation competence comparing pre-test and post-test. In these activities, the student develops self-care strategies identifying their impact on their well-being, allowing them to flourish in a daily environment. Well-being concepts are integrated for a full life, self-awareness, and self-care from positive psychology.

CONCLUSIONS: These Semana Tec activities aim to support the search for the fullness of the student considering human and professional growth. Semana Tec helps with the development of skills to define their life plan through real immersion experiences.

DEVELOPMENT OF SELF-AWARENESS COMPETENCE THROUGH EDUCATIONAL IMMERSION ACTIVITIES
Miriam Turrubiates | Mary Ana Cordero I Silvia Olivares

Tecnologico de Monterrey

PURPOSE: The development of self-awareness as competence is intended for the students to manage their practices for self-assessment, metacognition, and reflection. Selfawareness is essential for the development of personal and professional well-being, which is related to the possibility of establishing effective personal and professional relationships. Personal and professional well-being must be included as part of the academic training programs. For this reason, universities have the challenge to integrate self-awareness as part of the development of transversal competences. At Tecnologico de Monterrey, Semana Tec has been designed as an immersive action learning week that aims to contribute to the development of several skills. The present study aims to identify the development of Self-Awareness according to the Expectations Confirmation Theory, which compares students' expectations vs. final perception of the activities "Women's Leadership Bootcamp", "Activation energy" and "My today's selfie".

METHODS: A quantitative, descriptive, and transactional method was applied. A questionnaire was developed and implemented as a pre-test and post-test. This instrumente was validated by a focus group and the Cronbach's alpha resulted in 0.97. Twenty seven students answered the survey. For statistical analysis, the Wilcoxon test was used. RESULTS: The activities "Women's Leadership Bootcamp", "Activation energy" and "My today's selfie" had a significant statistical difference in Self-Awareness competence comparing pre-test and post-test. Students were able to establish a concept of themselves, describing their life from different approaches and perspectives. Self-awareness should be promoted as one of the well-being dimensions.

CONCLUSIONS: Semana Tec supported students in their human and professional growth, where the development of competences such as self-awareness was favored. The immersive action learning experience introduced the students on their self-assessment and individual attributes to enhance their personal and professional potential.

\section{INTEGRATION OF NEUROANATOMY, NEUROIM- AGING, AND NEUROLOGICAL CASES FOR NEXT GENERATION PHYSICIANS}

Lane Fortney | Sarah Garner I Richard Gonzalez I Alberto E. Musto

Eastern Virginia Medical School, Norfolk VA, 23507, USA

I Tulane University, New Orleans, LA, 70118, USA 
PURPOSE: As neuroanatomy knowledge only grows in complexity and clinical significance, medical curricular reform inversely marginalizes its integration. Similarly, with advances in medical imaging and educational technology, efforts to identify optimal and valid assessment of integration are inconsequential to novelty. Our goal was to investigate the integration of neuroanatomy through neuroimaging and neurological cases with considerations for the propitious preparation of next generation physicians and the future patient population.

METHODS: A literature review was utilized to identify the alignment of educational theories and principles of the neurobiology of learning for optimal neuroanatomy integration. The integration of anatomical structure, function, variation, development, and clinical application was evaluated across traditional educational activities and modern approaches. Parallels were derived from various integrative framework designs highlighting areas for discussion and recommendations of best practices for facilitating neuroanatomy integration.

RESULTS: Through the lens of the cognitive theory of multimedia learning, experiential learning theory, and motivational learning theory, integrative educational frameworks are optimally delivered when most closely resembling the human cognitive architecture. The integration of neuroanatomy through neuroimaging and neurological cases may provide a path for shaping integrative educational frameworks that promote collaborative efforts between medical education and advances in patient care through medical diagnostic technologies. Nonetheless, implementation is dependent upon the medical curriculum, institutional resources, and faculty training.

CONCLUSION: Efficient pedagogic integration of neuroanatomy through neuroimaging and neurological cases can enhance anatomical conceptualization and clinical application to advantageously equip future physicians and augment future patient care.

\section{ADDRESSING MENTAL HEALTH ISSUES ASSOCI- ATED WITH THE COVID-19 PANDEMIC THROUGH THE DEVELOPMENT AND PRESENTATION OF A VIRTUAL WORKSHOP}

\author{
Melanie Longhurst | Andres Aragon I Amro Awad I Abhishek \\ Dhran I Luz Luna | Herb Janssen
}

Paul L Foster School of Medicine, Texas Tech University Health Sciences Center El Paso

PURPOSE: Medical students at Texas Tech University Health Sciences Center El Paso have developed and presented virtual workshops to the community in English and Spanish to discuss the mental health impact of the
COVID-19 pandemic. The students have also developed brochures including mental health resources and tips to aid in the success of this project.

INTRODUCTION AND METHODS: The COVID-19 pandemic has impacted mental health by increasing stress, irritability, fear, confusion, anger, frustration, insomnia, depression, and anxiety. These emotions are triggered by experiences such as the loss of loved ones, financial uncertainty, work stress, isolation, increased substance abuse, and domestic violence. People who have contracted the SARSCoV-2 virus may also suffer from a direct mental impact of the infection. The need for a safe and rapid approach to address the mental health crisis it is critical. With the assistance of faculty members, students have successfully developed and presented informative virtual workshops that increase awareness of mental health issues and provide meaningful tips and resources to help address this crisis. Brochures and booklets are being developed to help distribute the information to those who lack Internet access.

RESULTS: This workshop has been presented to over 1,000 individuals in the local community and at other universities. Response from participants has been positive, suggesting this is a viable tool that can be used to help call attention to the current mental health issues and offer useful resources to attendees.

CONCLUSION: The COVID-19 pandemic has had a significant impact on mental health in the local border community, nationally, and globally. The need to safely discuss these issues remains a challenge. Students have successfully addressed this challenge using a technique that can be duplicated in other institutions.

\section{ASSESSING CURRENT APPLICATION REQUIRE- MENTS FOR RESIDENCY TRAINING PROGRAMS IN CANADA}

Sean Nurmsoo I Mohamad Besher Ourfali I Michael Bezuhly

Dalhousie University

PURPOSE: In recent years, some Canadian medical students have reported that Canadian residency programs have begun requiring proxies for academic ability from residency applicants. This requirement may be in response to a shift in Canadian medical education toward pass/fail grading systems, making differentiation between applicants on the basis of academic performance more difficult. With no Canadian equivalent to US Medical Licensing Exam scores, we sought to understand how commonly premedical proxies for academic ability are being used in lieu of medical school grades.

METHODS: We built a database of residency program application requirements using data retrieved from the 
Canadian Residency Matching System website between May 1st and July 15, 2017. We analyzed the proportion of residency programs requesting each application item. We then calculated both the proportion of residency programs and residency positions that require submission of a proxy item to apply.

RESULTS: After weighting by available positions in each program, we found $31 \%$ of Canadian residency positions required submission of pre-medical records in order to apply. Of the 514 residency programs we identified in Canada in 2017, $28 \%$ of programs required pre-medical records from applicants. This requirement varied widely by specialty and institution. 13 of 15 ophthalmology programs required results of a comprehensive eye exam conducted by an ophthalmologist, and 2 otolaryngology programs required or strongly recommended its inclusion. One program required applicants' MCAT score.

CONCLUSION: Requirements to include a premedical records may be an unintended consequence of the widespread shift in Canadian medical education away from numerical and letter grades. This may provide a preview of the United States residency match if USMLE exams become pass/fail.

\section{Student Support}

\section{INSIGHTS OF HEALTHCARE STUDENTS' DIGITAL CAPABILITIES}

Kwang Meng Cham I Mary-Louise Edwards I Lisa Kruesi I Tania Celeste I Trent Hennessey

\section{University of Melbourne, Victoria, Australia}

PURPOSE: Healthcare students value and recognize the key role digital literacy has in enhancing their employability. However, it remains questionable if students' everyday technological skills do accurately reflect their digital skills capabilities in learning environments.

METHODS: We evaluated the needs, practices and attitudes of students in the digital environment. Students' perceptions and preferences relating to digital technology ownership, usage, and computing experience was studied. Undergraduate (Oral Health) and postgraduate (Optometry, Physiotherapy, Speech Pathology and Dental Surgery) students across all year levels attended a digital literacy workshop and completed pre- and post-surveys.

RESULTS: Of the students $72 \%(n=343)$ reported using online tools several times a day, with Facebook (95\%) being most frequently used. Approximately 50\% wanted to learn how to create applications, websites, and 3D printing. Most of the students $(81 \%)$ reported that being digitally competent will enhance their career and professional development. Only $35 \%$ felt that this was achieved during their course of study, and $89 \%$ stated more University support and services is warranted.

CONCLUSIONS: Discipline specificity and workplace sector play an influential part in students' selection and use of tools and technologies and the range of digital capabilities they will develop (be required to develop) for transferring into the workplace. Higher education institutional structures and systems in partnership with workplace demands are strong drivers for students' digital capabilities around tools and technologies. With system upgrades across universities and the release and implementation of findings from new research in digital technologies, digital capability building for students will change to meet demand. These changes will require strong scaffolded support from across universities.

\section{REFLECTIVE PORTFOLIOS: A UNIVERSITY INI- TIATIVE TO PROACTIVELY SUPPORT PRE-CLIN- ICAL STUDENTS' REFLECTIVE PRACTICE}

Deborah Russ, PhD

West Virginia School of Osteopathic Medicine

PURPOSE: Because of past successes, many first year medical students do not feel the need to engage in reflective practice and are hesitant or unwilling to reach out for support when they encounter academic or personal challenges. This abstract describes a program designed to support students by incorporating regular reflections into appropriate clinical courses followed by a meeting with an assigned faculty coach to assess current academic and wellness strategies and set goals for positive change.

METHODS: All first year medical students wrote a guided reflection and met with their assigned faculty coach twice each semester. All second year medical students wrote a guided reflection and met with the same Year 1 coach once in the Fall semester and with their assigned Year 3 and 4 Regional Assistant Dean once in the Spring semester. Students addressed academic progress, wellness and the challenges of medical school in the reflections. All students completed a feedback survey at the end of each year. Faculty will complete a survey in December 2020 after the first full feedback cycle.

RESULTS: Initial student survey results indicated that $79 \%$ of students felt the reflection process was helpful to their academic progress. Over $96 \%$ of respondents felt their faculty coach was attentive and supportive, that their faculty coach had helpful suggestions regarding their experiences, and indicated the meetings were beneficial to their academic progress. Final student and faculty results will 
be collected in December 2020 and included for the 2021 IAMSE meeting.

CONCLUSION: The majority of students felt the reflection and meeting with a faculty coach was beneficial to their academic progress. Instilling a habit of reflective practice in the early stages of medical education could lead to improved outcomes and greater personal awareness throughout ones' career.

\section{PATTERNS OF EXTERNAL RESOURCE USE AND PERFORMANCE}

Uzoma Ikonne | Adrienne Brodie | Curt Bay | Anna Campbell

Eastern Virginia Medical School I A.T. Still University

PURPOSE: Understanding student resource use and selection can be informative to instructors and may guide curricular development and student advising. The purpose of this study is to compare the study resources students rely on in their first- and second-year academic courses, to determine the degree of influence various sources have on resource selection, and to determine the frequency of use of external resources for specific disciplines. We hypothesize that resource use may vary based on the level of academic performance.

METHODS: A survey was developed and sent to first- and second-year medical students in the fall semester. Survey responses were summarized and linked to academic and board performance measures.

RESULTS: First-year students more frequently used institutionally provided resources, relied more frequently on resources provided by instructors (i.e., Instructor slides, recorded presentations, practice questions), and reported more frequent use of self- and peer-generated resources. Second-year students indicated more frequent use of external resources such as products for board review and external resources for specific disciplines. Second-year students reported higher use of external resources when seeking efficient means of comprehending concepts when seeking effective learning resources. We did not observe a correlation between the use of external resources and COMLEX level 1 scores.

CONCLUSION: The second-year students more frequently use external resources compared to first-year students. Conversely, first-year students use resources provided by the instructors more than the second-year students. The use of external resources does not correlate with GPA or COMLEX scores. Future studies could expand on the current work by investigating resource use in clinical years of the curriculum and in residency, further investigate the perception of resources by students, and evaluate the manner in which external resources are being used.
THE SARS-COV-2 PANDEMIC'S IMPACT ON USMLE TESTING: MEDICAL STUDENT PERSPECTIVES REGARDING THE NBME AND PROMETRIC'S RESPONSE DURING THE PANDEMIC

Genna Bonfiglio

University of Colorado School of Medicine

PURPOSE: United States Medical Licensing Examination (USMLE) scores are critical to medical students' residency applications in the United States. During the SARS-CoV-2 Pandemic, the National Board of Medical Examiners (NBME) and Prometric, the company which provides testing centers, took 62 days to release a plan for delayed examinations. We aimed to determine student satisfaction with this response to pandemic-related rescheduling, additional student impacts, and opinions regarding how examinations could have been better adjusted during the pandemic and can be modified in the future.

METHODS: Medical students $(\mathrm{n}=570)$ from 21 medical schools completed our survey. We analyzed 483 survey responses based on a survey completion threshold of $50 \%$. RESULTS: Five percent (25) of surveyed students $(n=456)$ felt the NBME adequately responded to the pandemic. Seventy-three percent (350) of students $(n=478)$ "strongly disagreed" that Prometric provided adequate services during the pandemic. Qualitatively, students describe distrust of these entities, and negative mental health consequences. After scheduling delays, 69\% (326) of students $(n=476)$ felt "very stressed" about USMLE examinations, compared to 3\% (15) at baseline. 67\% (323) of students felt scheduling delays impacted exam performance "negatively" or "very negatively." Ninety-two percent (420) of students $(n=456)$ felt that medical schools should have been allowed to administer USMLE examinations earlier in the pandemic; $92 \%$ (419) of students $(n=455)$ felt medical schools should administer them long-term.

CONCLUSION: Given the impact that delayed exam rescheduling had on students, the NBME must reconsider USMLE administration logistics, Prometric's contract, medical schools as testing sites, and avenues for medical students to become active stakeholders in standardized board examination administration. US medical educators may offer additional support to students affected by these events. Medical educators outside of the US may wish to examine their respective standardized board examination procedures, and if students are considered stakeholders in this type of evaluation.

WELLNESS AT CARLE ILLINOIS COLLEGE OF MEDICINE: DEVELOPMENT OF A NOVEL PEER MENTORING PROGRAM 
Samantha Houser I Rahul Ramanathan I Jacqueline Newman I Andrea Jake I Daniel Teraguchi

Carle Illinois College of Medicine

PURPOSE: Medical schools across the USA employ various approaches to address student wellness and combat burnout and depression. Approaches include wellness seminars and large-scale events such as retreats. At Carle Illinois College of Medicine, we introduced another dimension in addition to seminars and retreats: the Wellness Mentoring Program. This peer-to-peer mentoring program is a novel initiative that pairs student mentors with mentees to provide holistic support at the individual level.

METHODS: Upperclassmen medical students were recruited through an application and interview process to serve as mentors for a 1-year term. Underclassmen medical students interested in receiving mentorship were chosen by lottery. Pairs were created based on common interests, selfdescribed personality traits, and gender preference. Mentees, with the support of their mentors, complete semesterly surveys to assess their satisfaction in nine categories (physical, mental, emotional, spiritual, vocational, social, financial, innovative, and intellectual wellness). Pairs meet minimally once per semester to discuss survey results and formulate achievable goals based on the mentee's needs.

RESULTS: 10 mentors and 15 mentees are current participants in the program (average class size $=36$ students). Once IRB approval is received in Spring 2021, we aim to collect verbal feedback regarding mentees' experiences in the program.

CONCLUSIONS: Results show successful implementation of an individualized peer-mentoring program to complement the wellness curriculum at Carle Illinois College of Medicine. In the future, we intend to collect additional qualitative feedback from participants as well as quantitative survey data to continually ensure our wellness curriculum is tailored to students' needs.

\section{A BILINGUAL, VIRTUAL SARS-COV-2/COVID- 19 PREVENTATIVE MEASURES EDUCATIONAL WORKSHOP}

Sinthuja Devarajan I Blanca Hernandez I Aaron MurilloRuiz I Shalabi Sorathia | Valeria Urbina I Wendy Walker | Jessica Chacon

Department of Medical Education, Paul L. Foster School of Medicine, Texas Tech University Health Sciences Center El Paso, El Paso, TX 79905, USA I Department of Molecular and Translational Medicine, Paul L Foster School of Medicine, Texas Tech University Health Sciences Center El Paso El Paso, TX, USA I Graduate School of Biomedical
Sciences, Texas Tech University Health Sciences Center El Paso, El Paso, TX, USA

PURPOSE: Due to the COVID-19 pandemic, students had limited opportunities to engage face-to-face with the community. Awareness and adherence to preventive measures such as social distancing, sanitization, and mask wearing are correlated with controlling outbreaks. To help students engage with their community, a group of faculty and students at Texas Tech University Health Sciences Center El Paso (TTUHSC El Paso) Paul L. Foster School of Medicine (PLFSOM) developed the bilingual, virtual platform, El Paso-Health Education and Awareness Team (EP-HEAT). One EP-HEAT workshop "Importance of Mask Wearing and How to Make Masks at Home" addressed COVID-19 preventative measures.

METHODS: Our sub team consisted of five medical students and two faculty moderators. Students created bilingual content for the preventative measures workshop, including PowerPoint presentations, videos on mask making, and downloadable pamphlets for the EP-HEAT webpage. Students presented the workshop at virtual education fairs for school districts, academic institutions, and other community groups. The Mask Making content was evaluated using a post-assessment survey sent to the health education fair participants. The survey measured 1) the rate of participant awareness and knowledge about preventive measures to decrease the spread of the novel coronavirus and 2) whether participants were more likely to follow those preventive measures.

RESULTS: Ninety-two percent of attendees $(n=80)$ who completed the post-assessment-survey agreed that attending our workshop increased their knowledge of preventive measures that limited the spread of the coronavirus. In addition, 97\% of participants wore a mask and followed proper hand washing guidelines to abate the spread of the virus.

CONCLUSION: The EP-HEAT preventative measures workshop was an effective tool to increase public awareness of the use of masks and hand washing to reduce COVID-19 transmission. Ongoing virtual health education efforts may continue to increase dissemination of evidence-based information, especially regarding preventative measures.

\section{IMPACT OF MEDICAL EDUCATION LEADERSHIP ON MEDICAL STUDENTS}

Aimee Pugh-Bernard I Jaclyn Anderson I Bryn Launer

University of Colorado Anschutz Medical Campus

PURPOSE: Leadership is proposed as an important aspect for medical students within the LCME guidelines. Medical students can gain leadership experience from a variety of 
opportunities within education, research, and outreach activities. Further, medical students are encouraged to attain competencies within medical education for their future role as medical educators. We surveyed medical students involved with the development of the 'COVID-19 Clinical Focus' course at the University of Colorado School of Medicine to determine the systemic effects of leadership within medical education.

METHODS: A post-pre survey using free response and a Likert scale question format was implemented to survey medical student leaders who participated in COVID course and content development. Questions focused on their role as student leaders in course development and their confidence in taking on future leadership in medical education were included. Competencies that are vital to the success of the medical student beyond undergraduate medical education, such as medical education development, organization, execution and scholarship, were also assessed. The importance of this leadership opportunity for fourth year medical students during residency interviews were also included to determine career impact. Likert scale questions were analyzed with an independent samples t-test.

RESULTS: Taking on a leadership position in medical education positively impacted medical students. Students expressed the development of key leadership skills and medical education competencies. Additionally, students acknowledged that their involvement as leaders in medical education was of great interest to residency programs.

CONCLUSION: Early medical education opportunities enable students to gain valuable expertise in leadership and medical education competencies essential to residents and attending physicians. These opportunities are highly valuable as career building and allow students to experience academia in a different capacity. Overall, students assessed their involvement in medical educational leadership as crucial to their development as a clinician.

\section{FIXING BURNOUT, BUILDING RETENTION: A CLASSROOM APPROACH}

Ritcha Saxena I Enock Augustin I Naresh Macker I Sarah Zubair I Nicholas Moore I Ritwik Raj Saxena I Ananya Raj

Medical University of the Americas I Super Specialty Pediatric Hospital and Postgraduate Teaching Institute

PURPOSE: There is a high prevalence of burnout among medical students, more so because traditional medical education emphasizes a transfer of ever-expanding bodies of scientific and clinical information to students, imposing on students a need to memorize the surplus data -making them susceptible to burnout. To curb this, there is a dire need for innovations in content and pedagogy. We developed an algorithmic approach in the pathology classroom designed to evoke a dynamic environment, with high demands on team communication and leadership, requiring precise clinical reasoning and quick decision making under time pressure. METHODS: Students from semester 2 and 4 participated in this study. In addition to the traditional lecture-based instruction, the students engaged in an assignment of algorithm creation and utilization. Student feedback was assessed using a Maslasch Burnout Inventory-Student Survey. Students answered a 22-item questionnaire that evaluated the reliability and effectiveness of the strategy and its ultimate impact on student burnout.

RESULTS: The results of the feedback survey suggested a higher concentration span and reduced lassitude among students after the exercise. Students reported having enjoyed it as a fun-activity, while also reporting better comprehension and retention abilities.

CONCLUSIONS: Designing these algorithms as a fun activity offers an interesting and effective platform that helps foster creativity, innovative thinking skills and problem-solving capabilities while also increasing morale and fostering collaboration. The more creative the learners can be, the more camaraderie they feel, and the easier it becomes to develop skills that help them make better decisions as future physicians. The overall experience results in associating the classroom with positive feelings, leading to enhanced student performance.

\section{AN INVESTIGATION ON THE IMPACT OF RESOURCE UTILIZATION ON MEDICAL STUDENT BURNOUT IN THE PRECLINICAL YEARS}

\section{Callie Torres I Dr. Angela Pierce}

\section{Kansas City University—Joplin}

PURPOSE: Medical school is fast paced and requires students to juggle several different difficult courses while immersed in a new learning environment. Research has shown that one in two medical students suffers from burnout during medical school. Several different aspects of burnout during the clinical years of medical school have been investigated. However, no research thus far has investigated the possible connection of extracurricular resources usage to burnout. Students may undergo additional stress due to the high price of extracurricular resources, the difficulty of utilizing a resource, a resource not living up to its advertised potential, and inadequate preparation for examinations due to bouncing from resource to resource- all of which can contribute to burnout. This study aims to investigate what extracurricular resources medical students are using and the manner in which they are utilizing them during their preclinical years of study and correlate it with burnout level. 
METHODS: First and second year preclinical medical students at Kansas City University Joplin and Kansas City campuses were surveyed via an online platform about their extracurricular resource utilization and burnout levels as measured by The Maslach Burnout Inventory General Survey for Students, MBI-GS(S). The extracurricular resource survey examined which resources were utilized, the amount of time spent with each resource, which resources the student would recommend and not recommend to a classmate, how much of a financial burden the resource was, and if the resource was associated with symptoms of burnout.

RESULTS AND CONCLUSIONS: Data collection and analysis is currently underway. We hope to gain more insight into which extracurricular resources students use, the manner in which they are utilized, and the possible connection to burnout levels. Information gathered from this research project will be used to help guide future students on which extracurricular resources are most beneficial hopefully leading to a less burnout.

\section{ELECTRONIC RESIDENCY APPLICATION SER- VICE EASE OF USE MEDICAL STUDENT SURVEY}

Jessica Zimo | Ellen Ribar | Leah Furman I Kathleen Inman Fuentes I Alda Maria Gonzaga, M.D.

\section{University of Pittsburgh School of Medicine}

PURPOSE: Most medical residency programs in the U.S. use the Electronic Residency Application Service (ERAS) for applications. The investigators observed that ERAS causes confusion for many medical students and limited formal guidance is available. We developed a survey investigating how ERAS caused difficulty for residency applicants.

METHODS: This cross-sectional survey study used a 10-min web-based questionnaire with multiple-choice and open-ended questions. The University of Pittsburgh IRB approved the study. The survey was distributed to U.S. medical students applying to U.S. residency programs with ERAS in 2020 using convenience and snowball sampling at the investigators' home institution and other U.S. medical schools. The survey inquired about overall difficulty, specific difficulties, and resources used to answer questions about ERAS. Analysis included descriptive statistics and qualitative analysis.

RESULTS: Recruitment and data collection are ongoing. Preliminarily, 45 students from 4 medical schools responded. 39 (87\%) reported difficulty with at least 1 ERAS section. Every section had difficulty reported by at least 2 students. 96 specific difficulties were identified, 77 from multiple-choice responses and 19 in free-text descriptions. On a Likert scale, classmates (2.45/3, StDev 0.59) and online forums $(2.25 / 3$, StDev 0.52) were rated as the most helpful resources, compared to official ERAS materials (2.09/3, StDev 0.59) and medical school advisors (1.77/3, StDev 0.67).

CONCLUSION: Medical students reported substantial difficulty with ERAS and an unmet need for helpful formal resources to guide them. The results of this study can be used to create a student-derived ERAS manual providing additional guidance for medical students from their peers.

\section{USING A SELF-REGULATED LEARNING FRAME- WORK TO STREAMLINE STUDENT ACADEMIC SUCCESS EFFORTS}

\section{Priyadarshini Dattathreya | Maureen Hall | Daria Ellis}

\section{Ross University School of Medicine}

PURPOSE: Lack of metacognitive and self-regulation skills has been identified as one of the academic factors that lead to poor exam performance among medical students. Medical schools provide academic success programs to develop metacognitive skills. These interventions can either be proactive (development oriented or deficit based) or reactive (deficit based). Our university provides a combination of these types of interventions to support our student success. In order to provide structure and consistent messaging across our programs, we needed a conceptual framework that was grounded in theory yet memorable and practical.

METHODS: We used the principles of self-regulated learning to develop a framework that provided an overview of metacognitive strategies employed before, during and after a learning event. We broke down the task of learning into seven discreet components to create the 7Ps framework. The components of the framework are Plan, Prepare, Participate, Process, Practice, Performance and Pause. To further support the framework, we developed a self-reporting inventory to help students reflect on their use of learning strategies, monitor progress and receive feedback for improvement. The inventory has helped us ensure continuity of support during transition to remote teaching.

RESULTS: The 7Ps framework has provided structure to our academic support activities. We use the framework to provide scaffolding in teaching metacognitive skills through academic success courses and workshops for remediating students. Students use the framework to set learning goals and develop academic action plans. We use feedback from the inventory to direct academic coaching conversations. We are also able to use the results from the 7Ps Inventory to identify areas where students need additional support resources.

CONCLUSION: The framework has helped us streamline our efforts to enhance student academic success and support the development of the AAMC Physician competency 
Practice Based Learning and Improvement even in a virtual environment.

\section{CASA: CAREER AND SPECIALTY ADVISING IN LEARNING COMMUNITIES}

\author{
Dr. Lisa Gilmer I Dr. Kelli Krase I Kaylee Story \\ University of Kansas School of Medicine
}

PURPOSE: This project seeks to develop formalized career and specialty advising access and delivery across campuses and specialties. Our goal is to strategically address career and specialty advising needs longitudinally at each stage of medical education to prepare students for the residency application process and match.

METHODS: CASA's creation began with a survey of the 2020 graduates about their experiences with the school's career and specialty advising. Next, 53 Specialty Liaisons from 21 specialties across three campuses were recruited and trained for their advising roles in CASA. In May 2020, third year students, specialty liaisons, program directors and recently matched fourth year students participated in virtual, specialty specific CASA kickoff sessions. As part of preclinical medical student career advising in 2020-2021, liaisons recorded Day in the Life interviews, participated in specialty interest group activities, and served as resources for the learning community coaches. For clinical medical student specialty advising, liaisons meet one-on-one with students starting in the spring of third year and continuing through the residency interview season. All CASA activities have easily converted to virtual formats facilitating tricampus participation of medical students and liaisons alike. RESULTS: Since the implementation of CASA, 156 students attended the kickoff sessions, and each of the 25 Day in the Life videos have received over 200 views. Data from the 2020 ISA and 2021 Post Match Data will be analyzed when available.

CONCLUSIONS: Continuous assessment of CASA will identify faculty development needs as well as unmet student advising needs. Thus far, preliminary data show that CASA has increased availability, quantity and quantity of career and specialty advising while also supporting our students whose shadowing and clinical experiences were limited due to COVID-19.

\section{AN INNOVATIVE METHOD TO AUGMENT MEN- TEES' INTEREST IN CLINICAL RESEARCH}

Amany Keruakous

University of Oklahoma Health Sciences Center
PURPOSE: The expansion of medical schools, faculty attrition, and trainees' disinterest in academia, highlight the need to encourage next generation of academic physicians. Early academic career awareness positively influence trainees' interest. Encouraging mentees is often difficult, due to time constraints, and work incentives. Which limits mentees' capabilities to participate in clinical research. Achieving equity among mentees in offering research opportunities within one institution is challenging. Moreover, the ease and availability of the opportunity makes it less valuable and increase the likelihood of mentees to lose interest. Establishing a direct gain and mentees' feeling "they merited the opportunity the hard way", affects the level of interest, and their perseverance to finish their research project.

METHOD: We developed an innovative way to engage our mentees in clinical research and augment their level of interest by forcing a phase of competition, having a direct profit for participation in research activity by earning authorship opportunities.

RESULTS: To augment mentees' interest in clinical research, an email with a task that is fundamental to start a research project was sent. The task is meant to consume 4-5 h. The subject of the email included a race sentence that offers a direct profit from participating promptly, "First 3 respondents will earn co-authorship opportunity". Email sent to nine mentees, four responded with their interest, they cooperated and finished the task the same day, maintained their interest and concluded their results on time. Sending the introductory email for clinical research to all mentees, including a direct profit for prompt response, forming a type of competition, resulted in more responses, persistent interest in research activities.

CONCLUSION: Mentees thrive to be the best, when all offered the same opportunity, thriving mentees dedicate their effort to accommodate the required task promptly when they earn the opportunity of co-authorship and maintain their enthusiasm to conclude their results.

\section{TESTING BEHAVIORS FOR INTERNATIONAL MEDICAL GRADUATES ON LICENSURE EXAMS}

\author{
Sherry Smith I Angela Hairrell
}

TrueLearn I Texas A\&M Health Science Center, College of Medicine

PURPOSE: One in four physicians practicing in the United States is categorized as an International Medical Graduate (IMG) and defined as a physician who received their undergraduate medical education outside of the United States and Canada (2000, NRMP). In 2020, the PGY-1 match rates for U.S. citizen IMGs and non-U.S. citizen IMGs were the 
highest since 1991 and 1990, respectively, with an overall match rate of $61 \%$ (2000, NRMP). Despite this increase, the match rates continue to be significantly lower than American Medical Graduates (AMGs) (2000, NRMP). Few studies have explored the study patterns of IMG. Diving deeper into the higher board scores for IMGs, this study aims to look at testing behaviors in a question bank as they prepare for licensure exams.

METHODS: Aggregate data collected by learners who have self-identified themselves as IMGs will be analyzed. Metrics that will be explored include the number of questions taken, overall performance, study schedule, test mode, and time spent.

RESULTS: Preliminary results suggest that IMGs take fewer questions in a more inconsistent pattern compared to their AMG counterparts. Additional analyses are currently underway to include other metrics.

CONCLUSION: In reviewing overall outcome measures, scores were comparable between IMGs to AMGs (Table 1). Conversely, when scores are broken down by specific specialties, scores for IMGs are higher than AMGs. Thirty percent of programs will never consider students with a Step 1 failure and 58\% will seldom consider (2018, NRMP), therefore, IMGs with multiple Step 1 attempts may not even be granted interviews by programs. This is another possible explanation for the lower match rate among IMGs. Results from this study will reveal study patterns and influence match trends for IMGs.

\section{CORRELATING STUDENT STUDY STRATEGIES WITH STUDENT SUCCESS, A LONGITUDINAL STUDY OF FIRST YEAR DENTAL STUDENTS IN BASIC SCIENCE COURSES}

\section{Kate J.F. Carnevale, Ph.D. I Algevis P. Wrench, Ph.D.}

Dr. Kiran C. Patel College of Allopathic Medicine, Nova Southeastern University

PURPOSE: The first year of dental school presents students with a heavy course load of challenging material, including several hard science courses, which necessitate that students quickly determine productive study strategies. In addition, for the Fall 2020 semester, the unprecedented shift to online learning, precipitated by the COVID-19 pandemic, engendered a new paradigm for the class of 2024 to adapt in order to be successful in their academics. We aim to investigate the correlation between study strategies and student success. METHODS: This work compares the results of self-reported individual student study strategy surveys with course assessment scores for those individuals as well as class averages for the 2019 and 2020 Fall courses of Biochemistry and Microbiology at Nova Southeastern University for the
Dental Doctoral classes of 2023 and $2024(n=131 \& 130$ students, respectively).

RESULTS: It is hypothesized that the best performing students in the class of 2023 will display a more typical pattern of a broad selection of study strategies for earlier exams, followed by a narrowing of successful approaches that will reflect better assessment scores due to students having identified what approaches work best at an individual level. It is also anticipated that the class of 2024 will have more sporadic approaches since they were not only learning how to study for a heavy course load but may also have struggled with the new modality and requisite self-discipline of onlineonly course content delivery.

CONCLUSIONS: Overall, it is anticipated that the class of 2024 will have experienced larger hurdles or a delay in establishing successful study strategies, as evidenced by poorer exam scores at representative time points, than the class of 2023. The results from this study will provide insight on better methods in advising students in best practices for effective studying and success in dental school and beyond.

\section{A COMPETENCY BASED CAREER DEVELOP- MENT PROGRAM FOR GRADUATE STUDENTS AND POSTDOCTORAL FELLOWS AT DUKE-NUS MEDICAL SCHOOL}

Sarada Bulchand I Silke Vogel

Duke-NUS Medical School

PURPOSE: PhD students and postdoctoral fellows in biomedical research face challenges such as dwindling positions in academia, deficiencies in career ready skill sets and lack of knowledge of career paths. Due to rapidly changing work environments universities need to develop programs that prepare students and postdocs for a VUCA (volatile, uncertain, complex, and ambiguous) world.

METHODS: With the establishment of the school's flagship Career Development Program (CDeP) we surveyed 48 $\mathrm{PhD}$ students and postdocs at Duke-NUS Medical School to inform program development. Based on the survey, a competency based curriculum centering on real world career skills, was developed. Five domains including communication skills, scientific skills, transferable skills, career planning and career experiences were developed via a 3-pronged approach of workshops, seminars and coaching. Awareness, skill building and reflective practices were built iteratively, in alignment with continuous feedback. Currently, Gibbs reflective cycle and program evaluation strategies are being utilized for program improvement.

RESULTS: Currently in its third year, CDeP has become integral to graduate education and also a support program 
for early career scientists/postdocs. CDeP has conducted 14 seminars to share career experiences across sectors, 16 workshops to build career ready skills and 30 coaching sessions to broaden mindsets, reaching over 600 participants who attended these trainings on a needs basis. Participant feedback indicates that training has been effective in building career awareness, knowledge and skills that can be applied irrespective of future career path.

CONCLUSION: Traditionally graduate students and postdocs receive only informal advice on best practices in career advancement. CDeP bridges the gap between the needs of early career researchers, and that of potential employers to ensure that students and postdocs are career ready and more confident of career transitions. The dynamic nature of careers in biomedical research highlight that a formal career development program is an urgent need.

\section{MEDICAL STUDENT ANXIETY LEVELS AND WELLNESS RESOURCE UTILIZATION}

David Lindars I James Ziegenbein I Kate Wigginton I Ara Alexanian I Valerie Gerriets

\section{California Northstate University College of Medicine}

PURPOSE: With the considerable rigor and stress of medical school, we have sought to better understand how medical students seek to address high/moderate anxiety levels by quantifying the utilization of counseling services and wellness events in medical students experiencing anxiety. This abstract describes the project and its outcomes.

METHODS: Wellbeing data has been collected over three years. A 20-min online survey has been provided to all medical students (first to fourth years) at the California Northstate University COM (CNUCOM) biannually. The survey includes demographic data, wellness events attended, and whether the student has seen a mental healthcare provider over various intervals. The bulk of the data are in the form of a Likert scale, or ordinal values, testing for levels of anxiety, burnout, etc. The anxiety quantifier used in the survey is the GAD-7 scale, the most commonly used self-reporting scale for generalized anxiety disorder.

RESULTS: 248 students have completed the survey. Of those who answered the survey, 21 of 248 students (8.47\%) reported high/moderate anxiety, and 15 of 21 (71.4\%) students with high/moderate anxiety attended either counseling or wellness events, with 9 of 21 (42.9\%) seeking counseling and 10 of $21(47.6 \%)$ attending wellness events. When isolating for help seeking in the form of attending wellness events, there is no statistically significant correlation with high/moderate anxiety level; however, there is a statistically significant correlation with attending counseling and high/ moderate anxiety level.

CONCLUSION: Determining how mental health services at medical school are utilized by students to either maintain or improve anxiety can encourage more educational institutions to provide such resources for their students, as more than $70 \%$ of students with high/moderate anxiety utilized wellness resources.

\section{THE NEED FOR BALINT GROUPS IN PHYSICIAN ASSISTANT EDUCATION}

\section{Lisa Waller}

MGH Institute of Health Professions, Physician Assistant Program

PURPOSE: Empathy, stress management, and emotional regulation have all been shown to be essential skills for healthcare providers. In many healthcare curricula, however, development of those skills is often assumed but not intentionally cultivated. Research also suggests a decline in empathy levels among healthcare students during clinical rotations. Balint groups were originally developed to provide regular, facilitated meetings among physicians to process difficult or unresolved feelings in the therapeutic physicianclient relationship. Could Balint groups be used among Physician Assistant students during clinical rotations to promote stress management and emotional regulation, and to mitigate against a decline in empathy?

METHODS: In 2019, Balint groups were introduced to a group of Physician Assistant students during clinical rotations as part of ongoing research. Three times over the course of the clinical year, while on campus for seminar week, students met in person in small groups for an hour. This discussion was facilitated by the Director of Clinical Education and another trained staff person. Students were asked to prepare in advance, to focus on interpersonal dynamics and feelings, and to present a case that left them unsettled or kept them up at night. As trust developed among group members, discussion followed about issues of end of life, conflict, family systems, addiction, diversity, or abuse. RESULTS: Student satisfaction surveys revealed that overwhelmingly, students found the Balint groups exceeded their expectations, were a good use of their time, and should be added to future clinical years.

CONCLUSIONS: Because of student feedback, Balint groups are now included as part of the required curriculum for future cohorts. Groups meet more frequently, at the end of each clinical rotation block. Further quantitative research would be useful in measuring empathy levels before and after Balint groups and into future practice. 
CONVERSATIONAL MEDICAL SPANISH COURSE: PROPOSED STRUCTURE FOR MEDICAL SCHOOLS

Sandra de Mel

Ponce Health Sciences University

PURPOSE: As the Latino population increases in the United States, medical schools face the challenge of better addressing population diversity in their curriculum. More than $50 \%$ of medical schools in the United States offer a medical Spanish course at their institution which indicates interest by students and faculty to better serve the Latino community. However, it has proven difficult to create an effective course that takes into account the limited extracurricular time of a medical student and the availability of resources at the institutions. In this abstract, we propose a novel structure for a conversational medical Spanish course.

METHODS: The course consists of in-class lectures, oneon-one mentoring, proficiency assessment tools, and standardized patient encounters. In-class sessions are one-hour, weekly sessions that are organized by organ system. The session is divided into a 30-min interactive topic presentation taught by a native speaker, a 10-min video of a patient encounter acted out by native speakers, and a 20 -min pairing of the student to a native speaker for an informal standardized patient encounter. Our pilot course will be launched in Spring 2021 and data will be collected for analysis of course effectiveness in improving language proficiency in the medical setting.

RESULTS: Initial interest surveys show this course structure is attractive to both medical school faculty and students. The aspects that most appealed to the students were the following: observing interactions between native speakers, one-onone mentoring by a native speaker, and more practice with standardized patients.

CONCLUSION: Creating a high-quality medical Spanish course is a difficult task. We have provided a student perspective on how to create the optimum conversational medical Spanish course that takes into consideration the best interests of the students and faculty.

\section{IMPACT OF A VIRTUAL MENTAL HEALTH FIRST AID ELECTIVE COURSE ON STIGMA IN FIRST- YEAR PHARMACY STUDENTS}

Himanshu Agrawal I Kevin Bozymski I Annabel Kuhn I Molly Schmidt I Wendy L. Peltier

Medical College of Wisconsin

PURPOSE: While pharmacy students have increased rates of anxiety and depression compared to the general population, many students report hesitation discussing mental health challenges with their peers. Therefore, our institution implemented an elective course that includes training in Mental Health First Aid (MHFA), a national certification program that teaches individuals how to recognize and respond to mental health crises. MHFA has been shown to decrease stigma in US pharmacy students and medical trainees from other countries; however, we are unaware of studies assessing the impact of a new virtual curriculum (MHFA 2.0) on stigma in health care learners.

METHODS: The elective course will be offered virtually to first-year pharmacy students. It will be co-taught by 2 faculty members (1 psychiatrist and 1 psychiatric pharmacist) who became certified MHFA instructors in November 2020. The program will have 3 components: First, students will complete the MHFA 2.0 curriculum that entails $2 \mathrm{~h}$ of independent prework and $5.5 \mathrm{~h}$ of live instruction. Next, students will attend a panel discussion led by individuals living with psychiatric conditions through National Alliance on Mental Illness (NAMI) programming. Finally, students will participate in small group discussions facilitated by psychotherapists. Students will complete the Opening Minds Stigma Scale for Health Care Providers (OMS-HC-15) before and after the course, as well as a written reflection after the NAMI programming, to determine the course's impact on stigma. RESULTS: Approximately 20 students will complete the course by the end of March 2021, after which OMS-HC-15 scores, written reflections, and course evaluations will be analyzed.

CONCLUSIONS: We anticipate this intervention will impact mental health stigma and justify further expansion of the virtual MHFA 2.0 curriculum into medical school curricula.

\section{IDENTIFYING ACADEMIC AND SOCIAL BARRIERS EXPERIENCED BY UNDERREPRESENTED STU- DENTS IN MASTERS AND OSTEOPATHIC MEDI- CAL PROGRAMS}

Angela Centeno-Gavica, MS, OMS-II I Gregory Haskin II, MS, OMS-II I Jewel Smith, MS, OMS-II I Ave Spencer, OMS-II । Tipsuda Junsanto-Bahri, MD | M. Esquire Anthony, DO

Touro College of Osteopathic Medicine

PURPOSE: In order to improve retention and progression of minority students, academic institutions need to understand various factors shaping their experiences in academia. Keeping the osteopathic philosophy in mind, it is important to apply the same thoughtful analyses to serve student bodies at medical institutions. The purpose of this study was to 
identify academic and social barriers faced by underrepresented minority students pursuing careers in medicine.

METHODS: A voluntary, validated, 22-item online survey was disseminated among MS, first-year, and second-year osteopathic medical students. The survey evaluated demographics, first-generation status, mentorship accessibility, barriers to undergraduate success, including experimental aspects such as feelings of belongingness as minorities in competitive academia based on a 5-point Likert scale ranging from strongly disagree to strongly agree.

RESULTS: 194 students were surveyed and 62\% $(\mathrm{N}=121)$ minority students were identified. Within this cohort, $46 \%$ identified as Asian, 21\% Hispanic, 18\% Black/AfricanAmerican, and $15 \%$ as other minorities. $67 \%$ female and $33 \%$ male. $79 \%$ were first-generation graduate/medical students. $60 \%$ did not have access to minority mentorship as undergraduates. Additionally, 58\% reported experiences of 'imposter syndrome'. Likert scale (agree, strongly agree) showed $93 \%$ felt anxiety related to academics, $80 \%$ felt their parents emphasized the importance of academic excellence, and $69 \%$ felt they worked harder to overcome additional barriers to obtain success equal to their peers.

CONCLUSIONS: Survey findings indicate high rates of inaccessibility to diverse mentorship during undergraduate education, including high rates of 'imposter syndrome' among minorities. Self-doubt, prospective anxiety, and burden of high expectations from social support were key factors identified among minority experiences. These aspects should be considered by academic institutions when designing programs and initiatives for minority applicant matriculation and success into medical programs. Further data is needed to explore how to address these findings and facilitate efficient use of university resources in enduring minority student success.

\section{EXPLORING STUDENT LEARNING ADAPTATIONS DURING THE COVID-19 PANDEMIC}

\section{Cortny Williams I Jenny Nordeen I Christopher Browne I Brent Marshall}

\section{University of Western States}

PURPOSE: Learning experiences abruptly pivoted from onground to wholly online in the COVID-19 pandemic. This study explores how students adapted their study space, study time, and approach to learning.

METHODS: Fifty-two of one hundred five students (49.5\%) in a graduate health sciences professional program participated in a survey to explore how shelter-in-place orders during the COVID-19 pandemic influenced their approach to learning.
RESULTS: Nearly all students described a challenge in adapting their study space, study time, or approach to learning. Students report that use of technology did not change because assessments and resources were electronic prior to the pandemic. Students who selected high impact study strategies like self-quiz or who demonstrated evidence of well-developed metacognition described a positive approach to adapt their learning more frequently than students who selected low impact study strategies like repeated reading or who did not show evidence of metacognitive development. CONCLUSIONS: Students with high-impact study strategies and well-developed metacognitive skills were more likely to make positive adaptations to the change in learning experiences brought on by the COVID-19 pandemic.

\section{TBL/PBL}

\section{USE OF A PROBLEM BASED LEARNING SES- SIONS IN DIVERSYFYING THE MEDICAL SCHOOL INTERVIEW PROCESS}

Uma Ramoutar I Samantha Marazita | Kyle Bauckman, Ph.D.

\section{Dr. Kiran C. Patel College of Allopathic Medicine}

PURPOSE: Medical school interviews focus on the ability to engage in a one-on-one conversation. Even modern techniques, like the Multiple Mini Interviews (MMIs) are based on closed conversational environments. The use of a Problem Based Learning (PBL) activity may serve as an indicator of a candidate's success in PBL driven curriculum. This method helps an institution dynamically assess the ability of an interviewee to interact with their peers. This allows the admissions committee to observe the interpersonal and professional skills of each candidate. This method provides candidates with an opportunity to determine whether they will be satisfied in a PBL driven curriculum.

METHODS: Candidates are invited to participate in the PBL session by the Dr. Kiran C. Patel College of Allopathic Medicine (NSUMD) admissions committee. Following the session, the candidates were emailed a link to an anonymous and voluntary survey. Candidates are gauged on interest and engagement in the PBL interview session and likeliness to attend a medical school with a PBL curriculum.

RESULTS: As of this submission, a total of 83 candidates completed the voluntary survey. $58 \%$ of participants had never participated in a PBL session prior to the session, 97\% of respondents felt interested and engaged during the session, $97 \%$ reported learning something new, $100 \%$ felt comfortable sharing knowledge with their peers, $80 \%$ reported preferring PBL over traditional interview format, $60 \%$ of 
participants felt the session provided them with an opportunity to demonstrate themselves to NSUMD admissions. 93\% of participants preferred attending a medical school with the PBL format.

CONCLUSIONS: Preliminary results show candidates find use of the mini PBL interview session interesting, engaging and prefer attending a medical school with a PBL curriculum. Future investigations will survey matriculating students on their views of the PBL interview and survey admission committee faculty on the utility of this data.

\section{THE IMPACT OF TEAM-BASED LEARNING ON THE CRITICAL THINKING SKILLS OF PHARMACY STUDENTS}

Dave Silberman I Rob Carpenter I Leanne Coyne I Jody Takemoto

Soules College of Business, The University of Texas at Tyler I College of Osteopathic Medicine, California Health Sciences University

\section{Abstract}

PURPOSE: Critical thinking is an important skill. Pharmacists are required to critically think throughout their careers. Despite efforts to develop critical thinking skills in a pharmacy school curriculum, few studies have found improvements in pharmacy student critical thinking. The purpose of this study was to evaluate the impact of team-based learning (TBL) on the critical thinking of pharmacy students. TBL is an active learning strategy that encourages students to think critically to solve problems.

METHODS: One hundred ninety students at a pharmacy school were invited to participate. The Health Science Reasoning Test (HSRT) was administered prior to the first semester of and after two years of the TBL-based pharmacy curriculum. The HSRT evaluates analysis, interpretation, inference, evaluation, explanation, induction, deduction, and numeracy. The Student t-test was used for a pairwise analysis along with Welch's t-test for unequal variances when comparing HSRT score modulation.

RESULTS: There was an overall increase in mean HSRT score and each individual HSRT domain demonstrated improvement that was statistically significant $(n=115)$. Nearly all (99\%) participants demonstrated improvements of one of the eight domains of critical thinking evaluated in the HSRT. However, some participants (29\%) with initially higher mean HSRT scores did not demonstrate an increase. CONCLUSION: Previous studies have demonstrated that TBL improves learner confidence in critical thinking but this is the first study that compares critical thinking skills prior to and following longitudinal TBL instruction using a critical thinking assessment test. This study provides evidence that TBL improves critical thinking skills. Students whose scores improved most had lower scores before initiation of TBL. Scores improved most in numeracy, analysis, and deductive reasoning. More students improved in inference, deduction, and induction than other domains. More research is needed to identify the specific aspects of TBL that influence critical thinking.

\section{THE CASE COVER SHEET-A TOOL USED FOR INTEGRATION DURING PBL TUTORIAL AT UNLVSOM}

\section{Rosalie Kalili}

\section{UNLVSOM}

PURPOSE: An integrated medical school curriculum requires planning and coordination by the various course directors and teaching faculty to ensure seamless execution and avoid missed opportunities for interweaving. We created a tool for Problem-based learning (PBL) tutorials that serves to facilitate discussion that incorporates concepts from the various courses of the curriculum.

METHODS: The preclinical curriculum includes various disciplines of medicine -Emergency response, Population health, Community outreach, Organ system-based basicscience, PBL, Clinical skills, Bioethics, Biostatistics, and Epidemiology. PBL comprises $20 \%$ of the curriculum, and its cases are written to reference material presented in all courses, enabling tutorial sessions to serve as a forum for interweaving. PBL Facilitators, who are clinicians in practice, need notification of the students' curricular activities in order to prompt and emphasize discussion that would allow for synthesis and application of concepts learned from the various courses. Thus, a concise graphic case cover sheet was created to summarize the students' weekly course topics for the Facilitators to review. A 9-item questionnaire using 5-point Likert scale was completed by the PBL Facilitators to evaluate the case cover sheet utility.

RESULTS: PBL Facilitators look at every weekly case cover sheet (4.5), agree that presenting students with opportunities to discuss concepts from various courses allows for application of knowledge that is relevant and meaningful to clinical practice (4.4), agree that being able to prompt discussion that incorporates various course concepts makes them better at their role as Facilitators (4.0), and agree that having a case cover sheet is valuable (3.9).

CONCLUSION: While the case cover sheet was easy to produce and intended to enhance purposeful interweaved discussion of the concurring course topics of the week, there were inconsistencies in the faculty Facilitators' use of the content provided. Faculty development on why and how to emphasize certain discussion would be beneficial. 
DEVELOPING AN INNOVATOR MINDSET (IM) IN MEDICAL SCHOOL LEADS TO TRANSFERRABLE SKILLSETS

Julia Schmitt | Kathryn Havens, MD | Ilya Avdeev, PhD | Karen Marcdante, MD I M. Chris Decker, MD

Medical College of Wisconsin

PURPOSE: The Transformational Ideas Initiative (TI2), a seed grant program run by the Kern Institute at the Medical College of Wisconsin (MCW), introduces faculty, staff and students to IM behaviors through project development activities. IM embraces the use of curiosity to understand problems, encourages connections/partnerships, and prioritizes the pursuit of value. We set out to understand if an IM skillset is beneficial to project development, and if participants would apply it to other professional activities. We hypothesized that IM is a skillset that transfers to other problem-solving environments.

METHODS: Throughout a series of four training workshops introducing IM, participants were coached through the development of their projects. Upon conclusion of the training, they rated themselves using an IM rubric according to the following dimensions: Curiosity: Identifying Stakeholders and Exploring Personas; Connections: Learning from Others and Presentation Skills; Creating Value: Identifying Value Propositions and Developing Hypotheses.

RESULTS: 2020-21 marks the third year of the TI2 program, with a current cohort of 73 faculty, students, and staff. The IM rubric survey response rate was $21 \%$. The average and range of the three rubric dimensions (possible ratings 1 (low) to 5 (high) revealed competency in: Curiosity: 4.60, 3-5; Connections: 4.44, 3-5; Creating Value 4.44, 3-5. All respondents report using IM in other parts of their clinical practice or class work, with $43.75 \%$ using the skillset more than once per week.

CONCLUSION: Participants reported that an IM is learnable frame of mind through skills training in the context of project development. They reported confidence applying the methodology in settings outside of the TI2 program. Our future goals are to measure behaviors of IM and how extensive the skillset is being applied in other programs and project development.

\section{DESIGN OF A MEDICAL SPANISH PROBLEM- BASED LEARNING CASE ELECTIVE}

\author{
Jacques Lowe I Krishin Shivdisani I Olivia Coiado \\ Carle Illinois College of Medicine
}

PURPOSE: The Carle Illinois College of Medicine utilizes Problem-Based Learning (PBL) as its primary learning modality for medical education. In recent efforts to improve students' cultural awareness, foreign language competency, and health disparities discussion, a PBL session was designed entirely in the Spanish language. The objectives of this study are: 1) to assess the Spanish grammar and vocabulary acquired, 2) to assess the usefulness and efficacy of a Spanish language PBL case and 3) to understand student's experiences with Spanish speaking populations.

METHODS: The PBL session is one week-long and will consist of eight medical students and one facilitator who will meet two times, $1 \mathrm{~h}$ per session, to discuss the case during Summer of 2021. The PBL case will be presented entirely in Spanish. Medical students can practice and assess their mastery of the Spanish language in a healthcare-oriented setting through the narrative provided in the case. Additionally, the facilitator for the PBL session will provide probes in Spanish to prompt discussion related to cultural awareness and understanding of the medical pathologies presented.

RESULTS: Students will fill out a pre- and post-survey that will employ a 5-point Likert scale. The surveys are intended to assess students' Spanish language competency as well as students' general understanding of healthcare from the point of view of Spanish speakers and the potential obstacles nonEnglish-speaking groups may encounter in the healthcare setting.

CONCLUSION: The addition of the Spanish Language PBL session shall provide a new avenue for medical students to explore, practice, and understand medical care from a Spanish speaking point of view. We foresee the implementation of this Spanish PBL case as a useful tool that will improve Spanish language skill and cultural awareness.

\section{THREE WEEKS OF TEAM-BASED LEANING DO NOT OVERLOAD UNDERGRADUATE STUDENTS}

Alexandre Lafleur I Mathieu Rousseau-Gagnon I Marianne Côté-Maheux I Dave Tremblay-Laroche I Paul René De Cotret I Yves Caumartin

Department of Medicine, Laval University Faculty of Medicine, Quebec City, Canada; CMA-MD Educational Leadership Chair in Health Professions Education I Department of Surgery, Laval University Faculty of Medicine, Quebec City, Canada

PURPOSE: Team-based learning (TBL) is a flipped-classroom approach requiring students to study before class. Fully flipped curricula usually have fewer in-class hours. However, for practical reasons, several programs implement a few weeks of TBL without adjusting the semester timetable. The literature on 
TBL does not provide clear indications if and how in-class time should be reduced when TBL is gradually introduced. Students fear that they will be overloaded by the individual and collaborative study hours needed to prepare for TBL. Our study aimed to address the gap between student concerns and empirical data. METHODS: We implemented three consecutive weeks of TBL in a fifteen-week lecture-based course on the renal system. In-class time and assessments were unchanged for all courses. 459 first-year undergraduate medical students (229 in 2018; 230 in 2019) were invited to complete weekly logs of their individual and collaborative study hours during lectures and TBL, along with questionnaires on cognitive load and perception of the course. Our program changed from A to E grading in 2018 to pass-fail grading in 2019.

RESULTS: Participants $(n=325)$ spent a similar number of hours studying for TBL vs. lectures with a mean of $3.1 \mathrm{~h} /$ week. Collaborative study was minimal outside class (median $0.1 \mathrm{~h} /$ week). Results remained similar with pass-fail grading. If in-class time were reduced, $18 \%$ of participants said they would have used freed-up time to study for TBL. Studying for TBL generated similar extraneous cognitive load and lower intrinsic load compared to studying for lectures; students were less stressed, and maintained high levels of motivation and self-perceived learning.

CONCLUSIONS: We replaced three weeks of lectures by TBL without reducing in-class time. First-year undergraduate medical students did not report overload in study hours or in cognitive load and maintained a good perception of the course.

THE MODIFIED TEAM-BASED LEARNING PROCESS TO FACILITATE ENGAGEMENT IN GROUP DISCUSSION

\section{Michael Sandak I Hye Yoon I Jaehwa Choi}

\section{Mercer University School of Medicine}

PURPOSE: The process of team-based learning (TBL) is as follows: (1) pre-class learning assignment, (2) individual readiness assurance test (IRAT), (3) group readiness assurance test (GRAT), (4) with or without mini-lecture, and (5) application activities. The effectiveness of learning through TBL activity was sometimes limited by several factors, including a lack of active participation by all group members but relying on a few high-achieving students telling the group the correct answer without meaningful team discussion. To enhance participation in the discussion by all of the group members, the modified TBL process was implemented.

METHODS: The in-class TBL activities were modified as follows: (1) group discussion based on the learning objectives and reading assignment, (2) IRAT, (3) GRAT, and (4) mini-lecture or application activities. Students' test results were compared with the previous class to evaluate the modified TBL activity's effectiveness in their learning.

RESULTS: Participation in group discussions was greatly improved. More reserved students asked questions to other group members so that they could do well in IRAT. The IRAT scores were improved, although they were not statistically significant. The test results were not significantly better compared to the previous class.

CONCLUSIONS: In summary, the revised TBL process effectively promoted group discussion by all group members. It was well-received by most students. However, the effect on the retention of their learning was inconclusive and needs further evaluation.

\section{EXPLORING LEARNING ISSUE RESOURCE UTI- LIZATION IN A PROBLEM-BASED LEARNING CURRICULUM}

Pavel Galchenko | Samantha Huang | Edward Liang | Margaret Burnett I Jaya G Yodh

Carle Illinois College of Medicine, University of Illinois at Urbana-Champaign, Urbana, IL 61801, USA

PURPOSE: Students at Carle Illinois College of Medicine build their pre-clerkship knowledge base in part through a problem-based learning (PBL) curriculum. Students use various resources to independently research learning issues (LIs) and present findings to their peers. Significant variation in depth and quality of LI presentations has led to this exploration of resource utilization with a goal of LI generation through enhanced LI framing and facilitator probes. Anticipated benefits include an increase in resource quality, depth of knowledge, and clinical practice impact.

METHODS: A five-member research team consisting of a PBL facilitator, medical librarian, and three second year medical students developed an LI-Reference Linkage Spreadsheet using data on 60 student-generated LIs and the corresponding resources used to research them. This deidentified data was extracted across preclinical curriculum from first and second year medical students in organ-based systems courses. A two-step independent rating process produced a consensus set of tags for systematically labelling LIs by discipline. A similar methodology was applied for categorizing resources used.

RESULTS: Preliminary tagging analysis of 20 de-identified LIs yielded a total of 35 consensus LI tags divided across 5 major categories encompassing basic, clinical, and social sciences, along with organ systems and engineering/innovation. Preliminary resource tags represent 7 categories based on assumption of quality, scholarship and evidence base: textbooks; journal literature; medical collections; 
government; consumer; commercial. Linkage patterns between the sources consulted and quality of tags are currently under investigation.

CONCLUSION: This study has enabled a robust tagging system for both LIs and corresponding resources. Preliminary results indicate that the tagging system can be used to guide evaluation of resource utilization and relative depth of knowledge acquisition in order to guide students' LI research. Future research will focus on LI-Resource linkage patterns and development of rubrics to assess resource quality and applicability towards improving learning outcomes in PBL.

\section{Technology and Innovation}

\section{HEALTH EDUCATION ADVANCES WITH LABORA- TORY SCIENCE (HEALS) WORKSHOP: A PILOT STUDY IN PERU}

Joanna Howard Ellis

Texas State University

PURPOSE: Widespread sharing of misinformation and misunderstandings about health have led to global trends like declining vaccination rates, underutilization of infectious disease testing, and inconsistent chronic disease management. I will describe a novel community-based health $(\mathrm{CBH})$ education approach that combines diagnostic screening tests with interactive, laboratory science-based activities to demystify health issues, prevention, and diagnosis.

METHODS: The Health Education Advances with Laboratory Science (HEALS) workshop began as part of a servicelearning study abroad program in Peru for undergraduates in a Clinical Laboratory Science program. I partnered with the Foundation for International Medical Relief of Children (FIMRC) to create the HEALS workshop as part of their ongoing CUY project. That program works with mothers and children in Aco to address nutrition challenges in the community. I collaborated with the FIMRC manager, local nurses, and physicians to determine community health concerns, screening tests needed, literacy levels, and local foods (ie. cuy, potatoes, rice). We identified IDA, diabetes, and diarrheal illnesses as common health concerns in Aco. The workshop consisted of a variety of culturally and linguistically appropriate CHE stations created to address the locally identified issues with scientific flare.

RESULTS: Following the first HEALS workshop, we surveyed mother participants. There were open-ended satisfaction questions and Likert scale knowledge questions. Ninety percent of respondents self-identified that their knowledge about IDA and diabetes significantly increased after the workshop $(\mathrm{p}=0.006$ and $\mathrm{p}=0.001$, respectively). All respondents stated that the workshop would help them make decisions in caring for their children and would help prevent future health problems.

CONCLUSIONS: Blending interactive science activities with a culturally appropriate, participatory, communitybased health education program may enhance understanding of public health concerns. This approach introduces families to instruments, concepts, and fields of study that may demystify health conditions and improve knowledge of the biological impact of dietary and lifestyle choices.

\section{THE RAPSODY OF VIRTUAL REALITY TEAM- BASED LEARNING}

Jody Takemoto | Rachel A. Bratteli | Brittany L. Parmentier I Thayer A Merritt I Leanne Coyne

California Health Sciences University I The University of Texas at Tyler

PURPOSE: The purpose of this study was to develop and implement the readiness assurance process (RAP) in a virtual reality (VR) environment and to evaluate the effectiveness of the RAP in a VR team-based learning (TBL) scenario.

METHODS: Participants were recruited through postings on the University campus and through student organization listservs. Students were provided with a short reading and escorted to individual VR stations to complete the RAP (e.g. iRAT, tRAT, appeals, and clarifying points). A survey collected participant demographic information and specific questions about their VR TBL experience on a 5-point Likert scale and open-ended questions.

RESULTS: Participants from diverse backgrounds and educational experiences participated in a RAP TBL experience. The majority of participants were White (30\%), Female (51\%), and/or between the ages of 18-24 years (89\%). The majority of participants responded positively to the experience indicating that they would take a VR TBL course $(83.33 \%)$ and would actually choose this environment over current online methods for team activities (88.89\%). A small percentage experienced vertigo $(29.63 \%)$ while participating.

CONCLUSION: This pilot study is the first successful demonstration of TBL conducted in VR. Opportunities for improvement includes minimizing vertigo while in the learning environment and improving use of this technology for team activities and assessments. Additional studies are warranted to implement a complete TBL module in VR from RAP through application exercises. 
TEACHING ANATOMY USING PEER-ASSISTED LEARNING IN AN ONLINE CASE-BASED LEARNING ENVIRONMENT

Harmandip Singh | Parvir Aujla | Jackie Nguyen I Amanda J. Chase I Stephen W. Ely

Dr. Kiran C. Patel College of Allopathic Medicine, Nova Southeastern University, Fort Lauderdale, FL 33314, USA

PURPOSE: Anatomy education in medical school curricula is in a state of perpetual evolution. Teaching modalities that have shown great promise for anatomy instruction include peer-assisted learning (PAL) and case-based learning (CBL). Our study assessed the effectiveness of implementing PAL and CBL in an online setting for anatomy instruction during the coronavirus disease 2019 pandemic.

METHODS: Third-year medical students $(n=3)$ held two online synchronous video conferencing sessions to teach clinically relevant cardiothoracic anatomy concepts to firstyear medical students $(n=54)$. The performance of the first-year students was evaluated using pre- and post-session multiple-choice quizzes. Additionally, material retention over one- and six-week periods was assessed using select questions from the first session. A post-session survey was also administered to assess student satisfaction with each session. Independent-samples t-test and Welch's ANOVA with Games-Howell post hoc tests were used to compare mean performance for dependent data. Paired t-test was utilized for the mean performance in the second session.

RESULTS: Mean quiz performance improved in the first session from $50.2 \%$ to $77.1 \%(\mathrm{p}<0.0001, \mathrm{~d}=1.570)$ and in the second session from $73.8 \%$ to $89.1 \%(\mathrm{p}<0.0001, \mathrm{~d}=0.808)$. There was a statistically significant increase in scores with the session one pre-quiz retention questions compared to the same session post-quiz ( $<<0.0001$ ), quiz one-week later $(\mathrm{p}<0.0001)$, and examination six-weeks later $(\mathrm{p}<0.0001)$. Student feedback was overwhelmingly positive with $79 \%$ agreeing that the activities improved their understanding of the material presented and $89 \%$ agreeing that their peers made valuable contributions.

CONCLUSION: This study demonstrates improved understanding and retention of the material as well as general student satisfaction through a combined PAL and CBL approach for teaching anatomy in an online setting.

\section{ACTIVE LEARNING ENVIRONMENTS: USING A DIGITAL WHITEBOARD IN AN ONLINE EDUCA- TIONAL MODEL AMID COVID-19}

\author{
E. Aniela Mendez Reguera I Mildred Lopez Cabrera \\ EMCS, Tec de Monterrey
}

PURPOSE: Pre-pandemic learning environments in face-toface formants were slowly transitioning to active-learning spaces focused on promoting interaction of students with each other and the teacher. Amid COVID-19, offering a dynamic and engaging online experience has been a challenge. In this abstract, we describe the implementation of a digital whiteboard to promote class engagement and an active-learning space.

METHODS: A digital whiteboard was implemented with Good notes app during synchronous sessions of an immunology class. Students collaborated in the construction of mind maps and process-diagrams while discussing the contents with peers and teachers. Students asked questions and provided comments on other students' input. These dynamics resembled the use of a blackboard as the one we had in the face-to-face format before shifting to an online setting. To assess students' perception of this innovation, an online survey was conducted. It considered a 5-point Likert scale, where 5 represents more favorable responses.

RESULTS: According to results, students did not feel class dynamics were affected as they perceived a comparable experience with both formats. Students reported they enjoyed drawing diagramas, and signaling or commenting over a digital version of their textbook as these diagrams helped them understand abstract concepts (4.83). They also highlighted that the inclusion of multiple learning resources kept them interested in the distance course (4.72), and that the teacher showed commitment on making a transition towards the distance model (4.94).

CONCLUSION: Using videoconferencing as the exclusive base of an online teaching experience will hardly substitute a face-to-face learning environment, but new technologies are a complement that can contribute to creating an active learning dynamic space for students and teachers interaction. By developing these environments, we contribute to bringing closer students and teachers to achieve a better experience during online classes.

\section{MOBILE APP FOR REAL-TIME ASSESSMENT OF PROFESSIONALISM IN PRE-CLERKSHIP MEDI- CAL EDUCATION}

James A. McAlister I Vijay Rajput, MD | Kyle Bauckman, $\mathrm{PhD}$

Dr. Kiran C. Patel College of Allopathic Medicine

PURPOSE: Professionalism is a core competency for all health professionals. The concept of professionalism is quite complex which is reflected by one's work ethic, performance, integrity, and motivation. These virtues require real time observation and assessment. Mobile medical education (mEducation) is becoming a new and frequent means 
of preclinical instruction. The dynamic nature of medical education begets real-time documentation of excellence and lapses in professionalism for immediate feedback. "ClassDojo" is a behavioral tracking and communication mobile app that is ubiquitously used in elementary education. This reporting system has shown positive outcomes in both communication with parents and student on professionalism expectations.

METHODS: ClassDojo has very limited presence in medical education. The application serves as an effective means of assessing medical students' professional attitudes and ethical behaviors expected in the classroom. We developed a protocol adapting ClassDojo apps in pre-clerkship education. We adapted anchors previously identified which are used in the learning environment. Students are assessed by determining number of opportunities missed vs total opportunities.

RESULTS: Preliminary data from ClassDojo suggests attendance and accountability as primary lapses in professionalism assessment in pre-clerkship courses. The mobility of ClassDojo allows real-time recording of events, formative coaching, and summative assessment of professionalism. CONCLUSIONS: Implementation of ClassDojo suggests attendance and accountability to be primary contributors toward undesirable professional behaviors in pre-clerkship curricula. The customizable nature of ClassDojo allows for versatility in any university teaching environment. Our preliminary use of the tool focused on professionalism observations via the course director. ClassDojo can be easily used in small group sessions including Problem Based Learning and clinical skills. This formative and summative assessment tool can be used for rapid assessment and remediation of recurrent professional lapses. Future directions will focus on developing strategies for optimizing ClassDojo and development of an app exclusively used for various clinical education settings.

\section{IMMERSIVE VIRTUAL REALITY (IVR) IMPROVES PROCEDURAL DURATION, TASK COMPLETION, AND ACCURACY IN SURGICAL TRAINEES: A SYS- TEMATIC REVIEW}

Randi Q. Mao I Lucy Lan I Jeffrey Kay I Ryan Lohre I Olufemi R. Ayeni I Danny P. Goel I Darren de SA

Michael G. DeGroote School of Medicine, McMaster University I Division of Orthopaedic Surgery, Department of Surgery, McMaster University I Department of Orthopaedics, University of British Columbia I Division of Orthopaedic Surgery, Department of Surgery, McMaster University I Department of Orthopaedics, University of British Columbia

PURPOSE: Immersive virtual reality (iVR) simulators provide accessible, low cost, realistic training adjuncts in time and financially constrained systems. With increasing evidence and utilization of this technology in training programs, its effect on global skill acquisition should be clarified. This systematic review examines the current literature on the effectiveness of iVR for surgical skills acquisition in medical students, residents, and staff surgeons.

METHODS: A literature search was performed on MEDLINE, EMBASE, CENTRAL, Web of Science and PsycInfo for primary studies published between January 1, 2000 and May 13, 2020. Two reviewers independently screened titles, abstracts, and full texts, extracted data, and assessed quality and strength of evidence using the Medical Education Research Quality Instrument (MERSQI) and Cochrane methodology. Results were qualitatively synthesized and descriptive statistics were calculated.

RESULTS: The literature search yielded 8,939 citations, with 13 articles included for qualitative synthesis. Immersive VR-trained groups performed $18 \%$ to $43 \%$ faster on procedural time to completion compared to control (pooled $\mathrm{SMD}=-1.11[95 \% \mathrm{CI}=-1.66$ to $-0.57, \mathrm{I} 2=1 \%, \mathrm{p}<0.0001]$ ). Two of four controlled trials that used task-specific checklists found iVR groups completed significantly more steps than control post-intervention. Four studies reported on implant placement accuracy. In two studies by Xin et al., iVR groups placed significantly more successful grade I/A pedicle screws compared to controls $(89.6 \%$ vs. $60.4 \%$, and $69.6 \%$ vs. $55.4 \%$ ). The mean MERSQI score was $11.88 \pm 1.60$. The strength of evidence for the outcomes 'time to completion' and 'implant placement accuracy' were rated 'moderate' on the GRADE framework, whereas the strength of 'task-specific checklist' and 'global rating scale' outcomes was rated 'low.'

CONCLUSION: Immersive VR incorporation into surgical training programs is supported by high-quality, albeit heterogeneous, studies demonstrating improved procedural times, task completion, and accuracy, positive user ratings, and cost-effectiveness.

\section{USE OF SPACE REPETITION SOFTWARE ON THE ENJOYMENT AND CONFIDENCE OF FUNDAMEN- TAL ANATOMY IN MEDICAL EDUCATION}

Heidi Kiziah I Summer Roorda I Nicholas Lutfi, MD, MS, DPM I Kyle Bauckman, PhD

\section{Dr. Kiran C. Patel College of Allopathic Medicine}

PURPOSE: Space repetition or "flash card" based study practices have long existed in academia. However, recent apps have developed sophisticated scheduling algorithms to augment this learning process. Anki is a popular app ubiquitously used and promoted on popular medical student forums. Though these decks have perceived benefits, content 
is not reviewed by experts and may deviate from institutional curriculum. We propose that institutionally driven Anki decks are beneficial for modern day learners. These decks allow students to practice basic concepts ahead of sessions and allow faculty to focus on broader, more nuanced concepts. We have piloted this process for our fundamental anatomy curriculum.

METHODS: Customized Anki decks were developed to directly represent the anatomy curriculum at the Dr. Kiran C. Patel College of Allopathic Medicine (NSUMD). These decks were mapped to the anatomy objectives in a collaborative effort between medical students and the chair of anatomy. Prior to the start of course, students were given a brief tutorial on how to use and best implement the institutional Anki decks. After completion of the course, students were surveyed anonymously to identify the utility of the Anki deck and its impact on student confidence and enjoyment of the material.

RESULTS: Students expressed positive feedback about the institutional Anki decks verbally and through survey response. Further, the initiative inspired students to collaborate with content experts in other fields to develop new decks.

CONCLUSIONS: Positive survey results support the creation and utilization of institutional Anki decks into other curricula at NSUMD. Spaced repetition tools will help facilitate the enjoyment and confidence while building a foundation of knowledge in a given curriculum. In turn, this increases student engagement in the preparation material allowing faculty to focus on higher order concepts.

\section{OBSERVATIONAL SKILL COMPETENCE USING EDUCATIONAL DISTANCE AND TELETRAINING}

\section{Rocio Casado I Santiago Celi I Matias Solari I Franco Funes | Guillermo Zalazar | Facundo Latini | Alberto Muisto}

Universidad Catolica de Cuyo, Argentina I Hospital de San Luis, Argentina I Eastern Virginia Medical School

PURPOSE: Acquire and assess observational skills competence using experimental clinical scenario through audiovisual media using an online platform.

METHODS: An educational video showing experimental seizures created at Eastern Virginia Medical School, Norfolk, VA, USA, were delivered to a group of medical students at the Catholic University of Cuyo, San Luis, Argentina, using online platform. Then, a session of training leading by principal educator to the medical students trainees, described different types of clinical seizures and its correlation with an assigned number. Using the video each student independently evaluated each seizures and compiled the results in a spreadsheet. This process was repeated three times every seven days, during which, the evaluators were blind to each other. Data was individually collected by research coordinator. Descriptive statistics were performed, and agreement was assessed by ICC (interclass correlation). RESULTS: Intraobserver between 0.85 and 0.97 for all evaluators between the three video review sessions separated by 7 days. Regarding the interobserver agreement, the result was between 0.80 to 0.90 with high repeatability, among the three evaluations. Each evaluator assessed the time to every RS stage and was compared to a scoring performed by a senior evaluator, showing a high correlation (adjusted R2 between 0.69-0.90). Intraobserver as well as interobserver agreement was assessed using the Intraclass correlation index (ICI) means with a 95\% confidence interval). The learning curve was compared using multiple linear regression.

CONCLUSIONS: The use of distant educational settings to acquire observation skills competences using a quantification analysis for its assessment showns to be a very useful tool, especially when training cannot be carried out in person or when collaborative clinical or research activities are required between distant places. We propose that this modality will be vitally important during and after the COVID-19 pandemic in order to instruct clinical exams in a large group of students.

\section{ADDRESSING SOCIAL DETERMINANTS OF HEALTH IN PATIENT DISCHARGES}

Radhika Sreedhar I Ananya Gangopadhyaya | Asra Khan | Ana Mauro

University of Illinois At Chicago

PURPOSE: Addressing social determinants of health (SDH) including housing, food security, and access to transportation are critical to ensuring a safe transition of patients from hospital to home. We share the results of implementing an innovative educational intervention that integrates instruction regarding SDH into a patient discharge curriculum.

METHODS: A four week patient discharge curriculum prepares fourth year medical students to safely discharge patients from the hospital. The recent Covid 19 pandemic and mitigation measures increased the emphasis on routinely enquiring and addressing social determinants of health as students discharge patients from the hospital. The educational intervention consisted of direct observation and feedback of patient discharges, post discharge phone calls and a small group discussion of how SDH impacts patient discharge. Pre and post tests were used to help assess the efficacy of this instruction in improving knowledge and skills in addressing SDH and student feedback was used to identify if this experience resulted in a change in attitude towards SDH. 
RESULTS: 63 fourth year students doing sub internship in medicine completed the pre-questionnaires and $67 \mathrm{com}-$ pleted the post questionnaires. There was a significant improvement in the proportion of students who reported moderate or greater awareness of how housing insecurity and access to transportation affect patients post discharge. There was also a significant increase in the proportion of students reporting that they needed little to no assistance in addressing housing insecurity, food insecurity and access to transportation. Student feedback revealed an improvement in self-reported student attitudes regarding SDH in post discharge care.

CONCLUSION: There is a dearth of literature on the effectiveness of curricula which integrate instruction of SDH in medicine with patient discharge. We present an intervention which improves medical students self-reported knowledge, skills and attitudes towards the role SDH in safe patient discharge.

\section{STANDARDIZING FEEDBACK FOR EVIDENCE BASED MEDICINE BASED SELF-DIRECTING LEARNING ACTIVITIES}

Radhika Sreedhar I Linda Chang I Ananya Gangopadhyaya I Amy Lin I Heather Heiman I Richards Maureen Hathaway I Chi Euna I Fernandes Christopher I Loza Julie I Jager Mathew David I Hanks Jessica Ryan I Sharkey Anser Carrie I Shiels Peggy Wozizodzki I Asra

University of Illinois At Chicago I University of Illinois at Rockford I University of Illinois At Peoria

PURPOSE: Health care students are expected to learn how to ask questions to answer their learning needs, effectively search medical literature, critically appraise it, and translate this knowledge to enhance patient care. Students need opportunities to help foster this curiosity, develop the skills of selfdirected learning and receive individualized feedback. We present a standardized approach and grading rubric to provide evidence based medicine (EBM) based individualized feedback for these self directed learning (SDL) activities.

METHODS: Students in pre-clinical years complete six written assignments where they identify a learning need in relation to a standardized patient they saw and create a foreground question. They are instructed to acquire an article of the highest level of evidence to answer this question, appraise it and comment on whether the results can be applied to their patient. We created an EBM based SDL grading rubric to enable evaluators to provide individualized feedback to students on this assignment. We present the results obtained by using this standardized assessment tool. RESULTS: Forty faculty members in three campuses were trained to use this SDL grading rubric. The results of interrater reliability of the rubric will be presented. The rubric has been used to evaluate over 300 students. We will present student performance in the areas of identifying a learning need, framing a clinical question, acquiring a highquality resource, appraising it and applying the results to their patient.

CONCLUSION: Students need opportunities to practice and receive timely feedback regarding EBM skills to become practitioners of evidence-based medicine. The standardized patient-based SDL assignments and grading rubric allow for ease of use for faculty grading and feedback in a longitudinal manner. This helps students develop the skills of self-directed learning needed for their clerkships.

\section{INTEGRATION AND RETENTION OF OSTEO- PATHIC PRINCIPLES AND PRACTICE THROUGH SIMULATION}

Kaitlyn M. Cedoz | William C. Grant | J. Gage Sanders | Paige M. Darrow I James R. Nolin I Dianne Walker I John T. Giannini

Department of Simulation Education, Alabama College of Osteopathic Medicine, Dothan, AL, USA I Department of Osteopathic Manipulative Medicine, Alabama College of Osteopathic Medicine, Dothan, AL, USA

PURPOSE: Simulation is gaining popularity in preclinical medical education across the world, including osteopathic medical schools. However, a literature search regarding the integration of osteopathic topics into simulation appears to be lacking. We believe that there is not currently a concrete incorporation of Osteopathic Principles and Practice (OPP) into simulation throughout the osteopathic medical school curriculum. We implemented a novel approach integrating OPP into simulation cases to determine if it improves student osteopathic knowledge and application.

METHODS: Medical students are randomly placed into teams of three for each curriculum-focused simulation case. They complete a form listing their top three differential diagnoses, physical exam findings from an osteopathic structural exam, and recommend appropriate osteopathic manipulative treatment (OMT) after finishing the case. The form is not required nor graded. Afterwards, students attend a debriefing session with faculty where a finding from the osteopathic structural exam, appropriate OMT, and a relevant osteopathic clinical application are highlighted. About one week later, students take a brief questionnaire regarding the last simulation case. RESULTS: While the study is still ongoing, it has provided insight into the benefits of simulation in osteopathic medical education. Students have managed to incorporate more osteopathic structural exams during the cases and then use these relevant findings to support their differential diagnoses. 
Students have increased the amount of OMT they would recommend for their patients and have shown an improvement in their retention of OPP since the initial case.

CONCLUSION: By further embedding OPP into simulation cases, the results of our study support that the integration of OPP into simulation reinforces students' knowledge while requiring them to apply it in a clinical setting. We plan to continue investigating the integration of OPP into simulation to further determine how much their OPP knowledge improves over-time with more clinical exposure.

\section{AFFORDABLE PHOTOREALISTIC 3D ANATOMI- CAL MODELS FOR MEDICAL EDUCATION}

\section{Daniel Rosen, MD MEd}

\section{University of Houston, College of Medicine}

PURPOSE: Access to adequate anatomical specimens in medical schools has been an increasing challenge. Here we describe a novel process for combining 3D printed anatomic models and photogrammetry that can be incorporated to any undergraduate or graduate medical school curriculum.

METHODS: Eight surgical and autopsy specimens were used to create 3D models including thyroid, larynx, colon, kidney, heart, lungs, brain, and liver. Photogrammetry images were acquired using the Trnio application on an iPhone XS max and postprocessed using Meshmixer software. The postprocessed file was used to produce a printed 3D model and a 3D image. 3D printing was performed on a 1:1 scale using white Polylactic Acid filament. In addition, the textured image file displayed on a smart tablet and projected on plexiglass sheet was placed at a $45^{\circ}$ to create the holographic illusion.

RESULTS: A minimum of 40 images (on average: 45) images were obtained for each model. The average acquisition time was $5 \mathrm{~min}$. Post-processing allowed exporting the model as a stereolithography format (.stl) for 3D printing and as a wavefront (.obj) file for 3D animation. Models were printed on 1:1 scale. The estimated cost was less than U\$S10 per model (U\$S 1.93 to U\$S9.27) and 6-28 $\mathrm{h}$ of print time depending on the complexity.

CONCLUSION: The 3D printed models displayed most anatomical features which are further enhanced by holographic reflection. It is feasible to create high resolution 3D anatomy models using smartphone devices and manipulate it to create didactic material. This technology may allow students exposure to similar learning material even in remote locations and may help understand complex anatomy and unusual anatomic variation. Although further studies are needed to assess the potential impact in medical student learning, this novel approach offers an affordable approach to provide 3D anatomical models to medical and other health profession students.

\section{USING EYE-TRACKING IN HISTOLOGY TO QUAN- TIFY PATTERN RECOGNITION SKILLS IN NOV- ICES AND EXPERTS}

Lauren Fitzgerald | John R. McMurray | Kevin W. Birdsall | Amelia Kkoo | Vasiliki M. Anemikos | Evan E. George | Robert J. Loving I Drew Levy I Caleb Haeussler I Bradley J. Schott I Srujan Kancharla I Heather Farmer Hoffmmann | Larry Johnson I Michael A. Paolini II I Ian V.J. Murray

Texas A\&M, Engineering Medicine I Texas A\&M, Veterinary Integrative Biosciences

PURPOSE: Students find histology challenging to learn, partly because it is a visual, implicit pattern recognition skill. While virtual histology slides have transformed education, further teaching innovations are needed. Eye-tracking can aid in the teaching of histology by identifying screening patterns involved in interpreting digital images. This converts an implicit skill into a quantifiable and observable task that can be demonstrated to students. The objective of this pilot study was to use eye-tracking data to determine if novices view histology images differently than experts. Few studies have utilized this technology to teach basic histology. METHODS: First and second-year medical students $(\mathrm{M} 1=4, \mathrm{M} 2=5)$ who completed a histology course in their first year of medical school were recruited for this pilot study and compared to 3 histology experts. Representative static images for 4 tissues were presented to the students for $5 \mathrm{~s}$ each. Using a webcam-based eye-tracking (RealEye.io), both eye and mouse movements were recorded and analyzed for patterns across representative tissues.

RESULTS: Quantitative analyses demonstrated that expert pattern recognition was characterized by longer fixations over regions of interest. This resulted in the scanning of fewer areas for simpler organs such as respiratory, and kidney cortex images, and scanning of larger regions for the more the complex ileum and sweat glands slides. Comparing the M1 to M2 indicated an increase in pattern recognition skill, and approached those of experts. Additional analysis of the number of fixations and total gaze scan lengths are currently being performed.

CONCLUSION: This pilot study demonstrates that an implicit visual skill can be converted to a quantifiable and observable task. The feedback from the eye-tracking information can be used to train novice medical students on how to identify key regions of interest and develop an effective pattern for approaching histological tissues. 


\section{EFFICACY AND BENEFITS OF AUGMENTED AND VIRTUAL REALITY BASED LEARNING DURING COVID-19}

Jessica Caruso I Lauren Harmon I Gabriella Born I David Dufeau, $\mathrm{PhD}$

Marian University College of Osteopathic Medicine

PURPOSE: The COVID-19 pandemic has created extensive disruptions for medical education, causing urgency to implement and develop solutions to combat this disturbance. For students to pursue learning opportunities, the education system must improve. Augmented (AR) and virtual reality (VR) provide a promising future for the enhancement of medical education. This review article aims to evaluate the benefits and efficacy of AR/VR, especially during the COVID-19 pandemic.

METHODS: Multiple peer-reviewed, randomized trials were synthesized into a review study investigating the efficacy, benefit, and use of AR/VR for medical education. The reviewed studies included medical and graduate-level students as participants and aimed to support the use of AR/VR as an educational adjunct. The results of these trials support the use of additional technology in medical education.

RESULTS: The COVID-19 pandemic propelled medical schools, educators, and students into a world of total computerized learning. This pandemic acted as a necessary push to implement novel, untested technologies into the educational sector. Previous research demonstrates that VR/AR can confront these unfamiliar challenges and allow for a safe and beneficial COVID-19 education, consisting of sociallydistanced and low-risk patient-care practice.

CONCLUSIONS: The benefits of continuous, safe learning outweigh the potential technological challenges of implementing AR/VR into medical education. During the pandemic, the medical community is faced with teaching students many skills necessary to become adept medical practitioners. Although the COVID-19 pandemic offers a unique opportunity for the implementation of new technologies, virtual instruction does not have to end beyond this crisis. Virtual technology can teach medical students interpersonal skills such as empathy, engaging in difficult conversations, and delivering bad news. Because students have traditionally been exposed to passive teaching styles, it is time to look toward expanding medical students' repertoire and allow them to personalize their education.

\section{SUPPORTING THE PATH TOWARDS A MEDICAL EDUCATION}

Jonathan Ho | Robert Dembinski | Zainab Balogun | Allison Mak I Daniel Pan
University of Pittsburgh School of Medicine

PURPOSE: The medical school applications process in America is one of the first barriers future physicians have to pass through in order to receive a medical education. While some can pay tens of thousands of dollars for professional support in this process, this possibility only disadvantages other medical school applicants who may already be struggling to afford the initial application fees. To tackle this issue while furthering an additional goal of supporting underrepresented applicants, we created a student-led, volunteer organization at the School of Medicine that provided free services for many of these applicants from a tristate region. METHODS: Our program was started in February 2020. In our first year, over 75 students at our medical school have volunteered to support with over 100 medical school applicants who have self-identified as being unable to afford paid services. Over the summer of 2020, these volunteers provided have comments on each of the students' tens of essays. And in the fall and winter, volunteers have provided mock interviews and provided further feedback on update letters to admissions committees.

RESULTS: Both applicants and volunteers have demonstrated their great satisfaction for our program thus far. And because most applicants will not hear back from schools until the spring of 2021, we hope to survey the applicants at that point to determine whether their rate of acceptance was higher than the $41 \%$ national average.

CONCLUSION: The positive feedback and our ability to continually expand to support more students within a tristate region demonstrates that even students at the start of their medical education can be impactful at making the application process fairer for disadvantaged students and diversifying the future physician population. Furthermore, our organization's model can easily be replicated to support even more students elsewhere.

\section{CASE BASED FLIPPED E-CLASSROOMS TO S.P.I.C.E UP THE E-LEARNING OF MICROBIOL- OGY AND INFECTIOUS DISEASES}

\section{Chitra Pai}

Touro University College of Osteopathic Medicine, Vallejo, CA 94591, USA

PURPOSE: The COVID-19 pandemic reiterates the importance of applied microbiology knowledge for the effective diagnosis, management, and control of infectious diseases. This project is an innovative pedagogy to spice-up the e-learning of Microbiology and Infectious Diseases using on-line Case Based and Team Based Learning approaches. 
METHODS: This innovative on-line pedagogy incorporates Harden's 'SPICES': Student-centered, Problem-based, Integrated, Community-based, Elective and Systematic approach. The test group consists of 270 preclinical students from Year-1 $(n=135)$ and Year-2 $(n=135)$ that are/will be taught infectious diseases using this innovative pedagogy. The control group consists of 270 students from the previous year, that were taught by conventional lectures. The Preclass work is asynchronous and consists of viewing short videos and solving practice quizzes. The Flipped e-classes are synchronous Zoom sessions employing interactive platforms including Poll Everywhere audience response system and the v3 InteDashboard platform. Each session is studentcentric with Case Based or Team Based learning formats. Student-satisfaction surveys and student-performance data from ExamSoft are used to assess project outcomes. Oneway ANOVA is used for statistical analysis of the results. RESULTS: In the Fall 2020 semester, 135 Year-1 students were taught by the innovative pedagogy. Students provided positive feedback and appreciated the opportunity to interact with their peers in teams and gain critical thinking and application skills in a highly engaging learning environment. By the conclusion of the Spring 2021 semester, additional data and results will be available from the Year-2 students. CONCLUSION: Lessons learned: This innovative project enhances the learning of infectious diseases by promoting higher levels of cognition and application. Short term benefits include better academic performances while long term benefits include skill sets for critical thinking and professional teamwork.

\section{AUGMENTING ANKI: GAMIFICATION OF SOFT- WARE TO PROMOTE RETRIEVAL PRACTICE WITH SPACED REPETITION}

\author{
Joseph Policarpio | Bailey MacInnis | Robert C. Wallon, PhD \\ Carle Illinois College of Medicine
}

PURPOSE: Anki is flashcard software aligned with the empirically-supported learning principles of retrieval practice and spaced repetition, used widely by medical students. However, users may struggle to prioritize its use due to its individual nature. To address this issue, we describe Battle Anki, an innovative software add-on for Anki which enables multiplayer capability. This design concept, known as gamification, adds game elements to traditionally nongame activities in order to help achieve learning goals by increasing motivation. Gamification is based on self-determination theory, which suggests learners may internalize external motivations by integrating autonomy and relatedness to colleagues. Battle Anki enables learners to study individualized material while competing in a low-stakes environment with a peer.

METHODS: Our team developed Battle Anki using Python and released the software on AnkiWeb and GitHub. In Battle Anki, any player can initiate a session with several options including the type and number of flashcards. If accepted by a partner, the study session begins for both players, and each player then completes their cards while viewing their opponent's real-time progress. The goal of the game is to study as efficiently as possible.

RESULTS: We aim to increase motivation and improve the efficiency of study time with gamification of Anki by providing social support that is not offered by Anki independently. However, we acknowledge the possibility that imposing competition and time constraints may lead to rushed or ineffective study, fatigue, or disproportionate overreliance on Battle Anki-which are outcomes that may interfere with the intended benefits of spaced-repetition retrieval practice and this add-on.

CONCLUSIONS: Gamification of Anki may help medical students increase intrinsic motivation, support social needs, and ultimately improve recall of medical knowledge. Further research on the use of this add-on is needed to investigate these potential outcomes, and to quantify and optimize such pedagogical benefits.

\section{PROFESSIONALISM AND HUMANISM IN TIMES OF PANDEMIC: ONLINE CLINICAL SIMULATION}

\author{
Mary Ana Cordero Diaz I Maria del Pilar Gonzalez \\ Amarante
}

Tecnologico de Monterrey

PURPOSE: Due to the SARS-COV2 health contingency and the cessation of face-to-face activities, a simulation exercise was redesigned and transformed to an online synchronic simulation via Zoom platform.

METHODS: The participating groups of medical students $(\mathrm{n}=53)$ were taking the Bioethics and Clinical Bioethics courses, adjunct to their Pediatrics and ObGyn clerkships in June 2020. Two simulated clinical cases were performed via Zoom in coordination with the Simulation Center, followed by a debriefing session. Later, an online survey was applied to the participants to know their perception and experience with this new version, considering they had experienced the original face-to-face simulation on the alternate clerkship the prior trimester.

RESULTS: The results show that the virtual format was very effective, $72 \%$ considered it very similar to the original version. The exercise revealed high emotional commitment, allowing students to develop their socio-emotional skills. Student reactions were categorized and coded as emotions 
triggered by a) their performance as professionals, b) those related directly to the patient's emotions and situation (which showed significant gender differences), and the least found c) students' anxiety related to the academic exercise itself. The fact that the clinical component was restructured due to the remote format may have helped in focusing on the preponderance of emotional, communication and relational aspects of the patient-doctor relationship. Also, most students identified the exercise was meaningful in approaching bioethics contents, including end-of-life decisions and care, informed consent, organ donation, among others.

CONCLUSIONS: In conclusion, the online clinical simulation activity proved effective in integrating professionalism outcomes that encompass ethical knowledge, skills and attitudes that prepare medical students for their professional role, along with the debriefing reinforcing insightful learning integration.

\section{STUDENT AND TEACHER EVALUATION OF AN ULTRASOUND TECHNOLOGY FOCUSED HIGH SCHOOL STEM OUTREACH PROGRAM}

\author{
Noor Akhter I Mohsin Syed I Karen L. Yanowitz I Gregory \\ R. Snead I Billy R. Thomas I Kevin D. Phelan
}

\section{University of Arkansas For Medical Sciences}

PURPOSE: ArkanSONO is an NIH funded STEM outreach program that brings state-of-the-art ultrasound and other imaging technology to 9 th grade classrooms to stimulate student interest and knowledge of STEM related fields and careers.

METHODS: The outreach program includes three separate 90-min visits to classes in the Little Rock School District. Here we report on our 1st outreach session to 50 separate Physical Science classrooms (2018-2019, 880 students). Sessions began with a short discussion of the properties of sound and principles of ultrasound. Students then worked in small groups using hand held ultrasound devices to scan structures in the neck and upper limb of the faculty. A large group session then summarized the lesson and asked students to apply their knowledge in a group application exercise. The session concluded with a general discussion of STEM careers and college. Students completed multiple choice pre- and post-session content knowledge surveys and a session evaluation survey that measured their level of engagement and perceived value of the activity. Teachers also completed a survey on their overall perception of the visits.

RESULTS: The student evaluations indicated that they were very engaged (average 3.1 out of 4 ) and found the session useful or valuable (average 4.7 out of 7). Nearly half of the 115 students who indicated they were "not at all" a science type of person rated the outreach session as having value/ useful (Likert ratings of 5-7). Teachers reported that more than $80 \%$ of their students were engaged, having fun, and came away with a positive attitude towards STEM.

CONCLUSION: Collective feedback from the students and teachers indicated that the ArkanSONO technology focused outreach program will serve as a unique model for instilling student interest and knowledge of STEM careers.

\section{DESIGN THINKING IN ACTION: A LEARNING ANA- LYTICS DRIVEN INTERPROFESSIONAL HEALTH HUMANITIES CURRICULUM TO ADDRESS SEX AND GENDER ISSUES}

Martha Eugenia Garcia-Osorio I Shirley Valentin Berrios I Sandra Chinapen-Barletta

San Juan Bautista School of Medicine

PURPOSE: This presentation describes one's institution experience with the development and implementation of a curricular innovation based in design thinking tools to face previouly identified needs: reinforcement of interprofessional education, diversity and inclusion in the curricula, and initiatives to promote wellness, humanism, and professionalism. The specific aim of the research project is to evaluate the effectiveness of the proposed curriculum, through learning analytics tools. The research questions being addressed are: a) Is a Health Humanities integrated curriculum useful to address required competencies in diversity and inclusion? and b) Are the data provided by learning analytics reliable to measure acquisition of competencies in diversity and inclusion in an integrated interprofessional curriculum in Health Humanities?

METHODS: The project includes: Curriculum database analysis for the sex and gender topics in 4 health professions programs. Identification of teaching / learning opportunities and instructional design. Alignment of the topics between the four programs. Curatorial practice of health humanities resources. Activities Implementation Learning analytics.

RESULTS: The curriculum inventory found that sex and gender topics were partially addressed in some educational activities of the programs, and that structured results about acquisition of competencies on those topics were not being collected and/or used. Multiple teaching/learning opportunities were identified and topics were aligned between the 4 programs. 305 people from 4 programs participated in the inaugural session. Individual reflections and interactive surveys highlighted innovation, relevance, interprofessional education and pertinence as main accomplishments. More than $95 \%$ of participants were highly satisfied with the activity. Experts in arts and humanities are suporting the curatorial process and a calendar of activities is already completed. 
Time constraint and logistic coordination have been the main challenges. Communication and team work have been key for the sucess.

CONCLUSIONS: This project is showing early signs of success for a new model that integrates humanities and professional readiness and can prepare students to navigate and effect change in today's complex world.
Publisher's Note Springer Nature remains neutral with regard to jurisdictional claims in published maps and institutional affiliations. 